Journal of Muscle Research and Cell Motility 6, 53-128 (1985)

\title{
Abstracts of the Thirteenth European Conference on Muscle and Motility
}

\author{
GWATT, 23-28 SEPTEMBER 1984
}

\section{GENE EXPRESSION, DEVELOPMENT AND DIFFERENTIATION}

The influence of muscular hyperactivity and hypoactivity on developmental changes of acetylcholine sensitivity of rat slow muscles G. Asmussen and U. Gaunitz

Carl-Ludwig-Institute of Physiology,

Karl-Marx-University, Leipzig, G.D.R.

Muscle fibres of newborn rats are sensitive to acetylcholine $(\mathrm{ACh})$ not only at the neuromuscular junction but also along the whole surface area of the fibres. The threshold of a contracture evoked by an application of ACh in the bathing solution on isolated muscle preparations may be taken as a measure of the extrajunctional ACh sensitivity. The assumption that the decrease of extrajunctional ACh sensitivity during early postnatal life is controlled by the activity of motor neurons supplying the muscle was tested in soleus muscle preparations of rats. As a model of motor neuronal hyperactivity served soleus muscles whose agonist (gastrocnemius muscle) was removed at the second day after birth. As a model of motor neuronal hypoactivity, soleus muscles of cordotomized and deafferented rats (cordotomy at Th 12 and cutting of the dorsal roots of the lumbar spinal cord two days after birth) were used. The muscles of the contralateral leg and muscles of untreated animals served as controls. Immediately after birth, normal soleus muscles show a contracture threshold of about $5 \mu \mathrm{M} \mathrm{ACh} \mathrm{l}^{-1}$ and this high sensitivity was unchanged up to the eighth day of life. Afterwards the sensitivity decreases progressively. Values characteristic of adult muscles (contracture threshold $5 \mathrm{m \mu} \mathrm{ACh}{ }^{-1}$ ) were reached around the 20th day after birth. Functional overload and hyperactivity of the soleus muscle by removing its agonist accelerates the decrease of extrajunctional ACh sensitivity of the muscle fibres during postnatal life. On the other hand hypoactivity by cordotomy and deafferentation has an opposite effect and retards the decrease of ACh sensitivity. The results stress the dependence of the properties of muscle fibres on the activity of motor neurons they innervate.

\section{Alkali myosin light chain gene expression in cardiac and fetal muscle \\ P. J. R. Barton, A. Cohen, P. Daubas, \\ M. Y. Fiszman, D. Leader, B. Robert and \\ M. E. Buckingham \\ Département de Biologie Moléculaire, Institut \\ Pasteur, F-75724 Paris cédex 15, France}

We have isolated clones from a cDNA library of mouse cardiac mRNA that encode part of the adult atrial $\left(\mathrm{LC1}_{\mathrm{A}}\right)$ and ventricular $\left(\mathrm{LC1}_{\mathrm{V}}\right)$ myosin light chains by cross-hybridization with the previously isolated adult fast skeletal $L C 1_{\mathrm{F}} / \mathrm{LC} 3_{\mathrm{F}}$ gene. In the mouse the fast skeletal isoforms $\mathrm{LCI}_{\mathrm{F}}$ and $\mathrm{LC}_{\mathrm{F}}$ are encoded by a single gene that is differentially spliced during maturation of the two specific mRNA types: the -COOH terminal region of these mRNAs is identical and is encoded by a common coding region with several distinct $-\mathrm{NH}_{2}$ coding exons specific to $\mathrm{LCI}_{\mathrm{F}}$ and $\mathrm{LC} 3_{\mathrm{F}}$. We show that at low stringency this common coding sequence can be used to detect other LC1-like sequences and have thereby isolated CDNA copies of cardiac LC1-like mRNA sequences.

One of these clones $(\mathrm{pC} 6$ ) contains part of the atrial $\mathrm{LCI}_{\mathrm{A}}$ sequence which hybridizes specifically to atrial but not ventricular or other adult muscle 
RNAs. This clone, however, shows hybridization to mRNA in 18 day fetal skeletal muscle that is of the same characteristic size and which shows the same thermal stability of hybridization as the atrial $\mathrm{LCI}_{\mathrm{A}}$ mRNA. pC6 also hybridizes to RNA from cultured T984-C110 myotubes and the in vitro transation product of this RNA comigrates with the fetal isoform $\mathrm{LCI}_{\mathrm{emb}}$ expressed in these cells. Two-dimensional gel analysis of adult atrial and fetal skeletal myofibrillar proteins shows $\mathrm{LCI}_{\mathrm{A}}$ and $\mathrm{LCI}_{\text {emb }}$ to be indistinguishable in the mouse. Hybridization of $\mathrm{pC} 6$ to mouse genomic DNA indicates the presence of a single genomic locus complementary to clone $\mathrm{pC} 6$ and we therefore conclude that the atrial light chain $\mathrm{LC}_{\mathrm{A}}$ and the fetal isoform $L C 1_{e m b}$ are encoded by the same gene and are identical.

The ventricular type cDNA clone pA29 has been identified as containing part of the $\mathrm{LCI}_{\mathrm{V}}$ mRNA sequence by northern blot hybridization and hybrid selected in vitro translation. As with the atrial $\mathrm{LCI}_{\mathrm{A}}$ we have shown that this sequence is encoded by a single genomic locus. Preliminary data indicate that $\mathrm{LC1}_{\mathrm{V}}$ mRNA is present in slow muscle fibres since it is detectable in soleus muscle containing mixed fast and slow fibre types yet is absent from RNA of fast skeletal muscle. Genetic analysis in the mouse shows that the genes coding for $L C 1_{A}, L C 1_{V}$ and the $L C 1_{F} / L C 3_{F}$ gene are located on separate mouse chromosomes.

\section{The expression of small nuclear RNAs is developmentally regulated in chick embryo myogenic cells \\ L. Carlsson \\ Department of Medical and Physiological Chemistry, BMC, Uppsala University, Box 575 , S-751 23 Uppsala, Sweden}

The synthesis and steady-state level of snRNAs have been analysed in various chick embryonic cells and tissues undergoing terminal differentiation. Chick embryo fibroblast cells constitutively synthesize snRNAs irrespective of DNA synthesis and mitotic activity. However, in in vitro cultured chick embryo myogenic cells, the relative rate of snRNA synthesis (U1, U2, U4-U6) decreases between 25- and 110-fold for the individual RNA species. This decline in synthesis is initiated 24-48 h after fusion of the myotubes is completed. No significant change in the halflives of the snRNAs has been detected. In contrast, tRNA and 5S rRNA synthesis decreases only 3-5 fold concomitant with a 10-20-fold decrease in the turnover rates, resulting in a net accumulation of these RNA molecules. Immunofluorescence microscopy using anti-Sm or anti-UI-RNP antibodies further supports the hypothesis that the expression of snRNPs is drastically reduced in chick embryo myotubes. Furthermore, there are no indications of a storage or stockpiling of snRNP-proteins in any other compartment of the cell (as has been suggested to occur for certain oocyte systems). Two other terminally differentiating systems were also investigated: chick embryo cerebellum and erythroid cells. In erythroid cells from various stages of the development of the embryo no apparent synthesis of snRNAs occurs, while in cerebellum a decrease in the synthesis is seen as concomitant with cessation of mitosis and initiation of terminal differentiation of neuronal cells. In the case of cerebellum, I have shown that the steady-state level of U2-RNA decreases about 25 -fold concomitant with the decrease in synthesis, by Northern blot analysis on total cerebellum RNA and a DNA clone specific for U2-RNA. In conclusion I have shown that the expression and synthesis of snRNPs are turned off in several postmitotic, terminally differentiating systems, including myogenic cells, and thus are developmentally regulated. I propose that this process is an important regulatory step in the reprogramming of the gene expression and turnoff of host cell functions which takes place during the final stages of terminal differentiation of various cell types in the chick embryo.

\section{Altered turnover of acetylcholine receptor in dystrophic mouse myotubes in vitro}

G. Cossu, F. Eusebi, M. I. Senni and M. Molinaro

Institute of Histology and General Embryology,

University of Rome, Italy

Multinucleated myotubes, developed in vitro from satellite cells of dystrophic mice (C57BL/6)/dydy), exhibit a reduced sensitivity to acetylcholine (ACh). This reduction correlates with a reduced density of ${ }^{125}$ I-bungarotoxin (BTX) binding sites on the surface of dystrophic myotubes. This finding was unexpected, since it is known that the sensitivity to the transmitter in the synaptic region of dystrophic muscle fibres in vivo is unchanged [Harris \& Ribchester, J. Physiol, Lond. 297, 245 (1979)]. Furthermore, denervated adult muscle fibres from dystrophic mice respond to $\mathrm{ACh}$ similarly to denervated normal muscle fibres. Cultured dystrophic myotubes, treated with a brain extract which induces acetylcholine receptor (AChR) clusterization [Jessel et al., Proc. natn. Acad. Sci. U.S.A. 76, 5397 (1979)], show a reduced 
sensitivity to ACh and reduced ${ }^{125}$ I-BTX binding. Thus, AChR appears altered in dystrophic muscle cell in culture while it appears normal in dystrophic adult muscle, regardless of whether the receptors are dispersed on the membrane or clustered at the junctional site. These data support the idea that different forms of AChR might be present during muscle development: an 'embryonic' form, expressed in culture, and an 'adult' form present in vivo [Pumplin \& Fambrough, Ann. Rev. Biochem. 44, 319 (1982)]; only the first form might be altered in dystrophy. Metabolic studies on the reduced $A C h R$ level is dystrophic myotubes revealed a dramatically reduced halflife ( 2 versus $10 \mathrm{~h}$ ) while the rate of synthesis was unchanged. An increased rate of internalization of $A C h R$ was observed in dystrophic myotubes with a concomitant relative increase of the 'hidden AChR pool', which could be partially reversed by agents which disrupt the cytoskeleton. No structural alterations could be shown in the AChR molecule as its migration coefficient and subunit composition appeared identical between normal and dystrophic myotubes.

\section{Immunogenetic analysis of human muscle cell surface \\ Roger D. Cox and Frank S. Walsh \\ Molecular Neurobiology Laboratory, Institute of Neurology, Queen Square, London WC1N 3BG, U.K.}

Interspecific muscle somatic cell hybrids promise to be of great value in the identification and genetic characterization of molecules involved in skeletal muscle development and differentiation. We are therefore using such hybrids as an immunogen to produce monoclonal antibodies ( $\mathrm{McAb}$ ) that define human specific epitopes at the cell surface. Human skeletal muscle myoblasts have been fused with a HGPRT negative clone of the mouse myoblast G8-I line and hybrids selected in culture medium. supplemented with HAT and ouabain. Hybrids were obtained at a frequency of $0.2 \times 10^{-4}$, and all clones tested were found to be myogenic. Hybrid clone RC4.J.1 grew rapidly and readily attains greater than $70 \%$ nuclei in well-differentiated myotubes, under conditions promoting fusion. This clone also expressed and regulated human muscle antigens at the cell surface. For example, muscle specific McAb 5.IHII was found on myoblasts and myotubes, while McA.b reactive with human Thy-I antigen was present on myoblasts only; these patterns of gene expression are similar to those found in human muscle cultures
[Walsh \& Ritter, Nature, Lond. 289, 60 (1981)]. RC4.J.I cells contained $96 \pm 37$ chromosomes at 64 days in vitro (DIV) indicating that they are the products of a $1: 1$ fusion since $\mathrm{G} 8-1$ cells contain 68 chromosomes. A muscle hybrid panel which had undergone extensive human chromosome nondisjunction was obtained by subculturing and cloning. At 259 DIV, subclone RC4.J.1.2.8 was shown by isoenzyme gel electrophoresis to contain only five human chromosomes $(X, 15,14,12$ and 7), the lowest number observed in the panel so far. Hybrid subclones are being used as a source of immunogen, in order to identify human gene products encoded by the remaining human chromosomes. To select $\mathrm{McAb}$ reactive with human but not mouse muscle cells, a differential ELISA assay was devised using either human skeletal muscle or somatic cell hybrid and G8-1 cells as targets. McAb A24 has a pattern of immunoreactivity similar to Thy-I antigen in cell culture but differs from it in cryostat sections. Five McAbs were found to react with antigens of the extracellular matrix and a number of McAbs show reactivity with cytoplasmic antigens. McAb D2AFI and DIB.C1I are cytoplasmic differentiation antigens whose expression is restricted to certain muscle fibre types. The biochemical nature of the above antigens is under study as is the identification of the chromosome responsible for their synthesis.

\section{Contractile and regulatory protein expression in the developing and adult human myocardium-adaptive mechanisms?}

P. Cummins, J. E. Humphreys, R. A. Janes and

S. J. Lambert

Molecular Cardiology Unit, Department of

Cardiovascular Medicine, University of

Birmingham, Birmingham B15 2TH, U.K.

The ability of the myocardium to respond to normal and abnormal physiological demands depends to an unknown extent on the isotype composition of its contractile and regulatory myofibrillar proteins. We have mapped the contractile protein isotopes of myosin (heavy and light chain subunits), tropomyosin ( $\alpha$ and $\beta$ subunits) and troponin-I, to investigate whether isotype expression is homogeneous throughout the human myocardium. Comparable studies were conducted in other nonhuman primate and large mammalian hearts. Myosin light chain isotypes differed in atria and ventricles in the adult and during development. Transitions between atrial and ventricular forms occurred at many developmental and adult ages and in certain cardiovascular diseases. Myosin 
heavy chain isotypes were less variable in the ventricle than in the atria with both developmental and pathological transitions. Tropomyosin $a$ and $\beta$ subunits varied in relative proportions in line with changing contraction speeds but troponin-I expression was homogeneous throughout the myocardium.

The pattern of contractile protein expression can differ markedly throughout the myocardium and between large and small mammals and may limit the physiological response in man.

\section{The role of the nerve in the expression of myosin heavy chain isoforms in a nerve-muscle tissue culture system \\ M. Ecob and R. G. Whalen ${ }^{2}$ \\ ${ }^{1}$ Muscular Dystrophy Laboratories, \\ Newcastle upon Tyne, U.K. \\ ${ }^{2}$ Institut Pasteur, Paris, France}

When several $1 \mathrm{~cm}$. long pieces of adult mouse muscle fibres are cocultured with embryonic mouse spinal cord, the muscle degenerates and regenerates to form a bundle of new myotubes. These myotubes, in the continued presence of the cord, differentiate morphologically into striated contracting muscle fibres. We have previously shown [Ecob et al., Differentiation 25, 84-7 (1983)] that cultures which have been contracting for 10 days or more contain the adult isoform of fast myosin heavy chain, usually in the large diameter fibres $(8-15 \mu \mathrm{m})$ which are presumably innervated. Using immunocytochemistry with antibodies to rat neonatal adult slow or adult fast myosin heavy chains, we have now shown that cultures with different levels of morphological differentiation (myotubes; striated myotubes; striated and contracting myotubes) contain different combinations of myosin isoforms, irrespective of their age in culture (7-28 days). Morphologically undifferentiated myotubes (at $7,13,17$ days) contain neonatal and slow but no fast myosin heavy chains. With the appearance of striations (at 13 days) fast myosin appears in fibres, usually in association with neonatal myosin and occasionally with additional low levels of slow. Large diameter fibres in contracting cultures contain either neonatal and fast or, at later times, fast alone.

If the nerve-muscle cultures are established and maintained in the presence of curare $\left(10^{-5} \mathrm{~g}\right.$ $\mathrm{ml}^{-1}$ ), there is a $4-7$-fold decrease in the proportion of fibres (compared with control cultures of similar age and fibre diameters) which contain fast myosin heavy chain.

If the muscle fibres regenerate in the absence of spinal cord, fast myosin is never expressed, even after equivalent times in culture (20-35 days), or in fibres of a similar diameter $(8-15 \mu \mathrm{m})$. These fibres always contain neonatal myosin with or without slow myosin.

\section{Human muscle $\mathrm{X}$-chromosome encoded expressed DNA sequences}

Micaela Fairman and Frank S. Walsh

Molecular Neurobiology Laboratory, Institute of

Neurology, Queen Square, London WC1N 3BG,

U.K.

We wish to identify specific X-chromosome sequences that are expressed in skeletal muscle. The characterization of such sequences is important for understanding the pattern of gene expression in normal tissues as a prelude to studying their expression in disease states such as X-linked Duchenne muscular dystrophy. A genomic $X$ chromosome specific library [Kunkel et al., Nucl. Acid Res. 10, 1557 (1983)] was screened on duplicate filters with ${ }^{32} \mathrm{P}$-labelled $\mathrm{cDNA}$ from human skeletal muscle, and human liver. Pretreatment of the CDNA with sonicated fetal DNA(100 $\mu \mathrm{g} \mathrm{ml}^{-1}$ DNA hybridized for $2 \mathrm{~h}$ at $65^{\circ} \mathrm{C}$ before adding to filters) significantly reduces the signal generated by transcribed, repetitive sequences, which are present in $10-25 \%$ of mammalian cell populations. Using this method, 64000 clones have been screened in duplicate and over 50 clones have been chosen for further analysis. After a second round of screening eight clones have been shown to exhibit higher signal hybridization on muscle fitters, while the majority of clones showed similar signal intensities with both tissues. DNA has been isolated from these eight clones and muscle CDNA shown to hybridize specifically to the human DNA after Hind III digestion. Insert sizes range from 0.6 to $4.6 \mathrm{~Kb}$. Each clone has also been hybridized against a dot blot of total RNA from human skeletal muscle and liver over a range of concentrations. Six clones give a weak or barely detectable signal which may indicate the presence of a low copy number or unique sequence. The other two clones give a stronger signal and one, phage MF572, when hybridized to a Northern blot showed the smear pattern characteristic of the presence of a repeat sequence. A further screen involving the absorbtion of both liver RNA and fetal DNA with muscle DNA, before hybridization against the $X$-library, also yielded a further eight clones not identified in the previous screen. These clones are being regionally assigned on the $\mathrm{X}$-chromosome to determine whether any are located at the $X_{p} 21$ 
region that is near to the Duchenne muscular dystrophy locus.

The genes coding for the $a$-subunits of skeletal and smooth muscle tropomyosin: structure and expression during muscle development

M. Y. Fiszman ${ }^{1}$, E. Kardami ${ }^{2}$ and M. Lemonnier ${ }^{1}$

${ }^{1}$ Department of Molecular Biology, Pasteur

Institute, Paris, France

${ }^{2}$ Department of Zoology, University of California,

Berkeley, California, U.S.A.

Affinity purified antibodies against $\alpha$-tropomyosin from chicken pectoralis major muscle recognize specifically the 43000 dalton tropomyosin component from gizzard $\left(a \mathrm{G}_{\mathrm{z}}-\mathrm{TM}\right)$. Limited tryptic cleavage provides evidence that these antibodies recognize sites located on the $-\mathrm{COOH}$ half of the $a-P M$ and $a-G_{z}$ tropomyosins. Using a cDNA which represents the $3^{\prime}$-untranslated region and $90 \%$ of the coding sequence of the mRNA coding for $a$-PM tropomyosin we have characterized the mRNA coding for $a-G_{z}$ tropomyosin which is a $2.0 \mathrm{~Kb}$ molecule. The $1.2 \mathrm{~Kb}$ mRNA ( $a$-PM tropomyosin) and the $2.0 \mathrm{~Kb}$ mRNA show greater than $90 \%$ homology over their $3^{\prime}$-coding region. However, the two mRNA have distinct $3^{\prime}$ untranslated regions. By screening a chicken genomic library we have isolated the gene which codes for $a$-PM tropomyosin and will show that it is linked to the gene which codes for $a-G_{z}$ tropomyosin. Lastly, we will present evidence that the two genes are coexpressed during muscle development. However, this level of expression is modulated so that in young embryos ( $7-8$ days in ovo) the two mRNA are present in equal amount while in older embryos the mRNA corresponding to the skeletal muscle gene is the major form. It must be noted that in muscle cells differentiated in vitro, the gizzard gene is not expressed. To try to understand the reasons for this coexpression, the 5 -ends of the two genes are being analysed.

\section{New sarcomere formation in myofibrils of mature skeletal muscle}

A. Jakubiec-Puka, J. Szczepanowska,

H. Chomontowska and J. Kordowska

Laboratory of Protein Metabolism, Nencki

Institute of Experimental Biology, U1.Pasteura 3,

02-093 Warsaw, Poland

During reorganization of the contractile structure of mammalian striated muscle, the number of sarcomeres changes within a few days [Williams \& Goldspink, J. Anat. 116, 45 (1976); McLachlan \& Chua, Neurosci. Lett. 35, 127 (1983)]. 'Extra sarcomeres' appear frequently in the course of such processes, most probably in the region of reorganization [Thornell, J. molec. cell. Cardiol. 5, $409,(1973)]$. We found an increase in the number of 'extra sarcomeres' in rat leg muscles maintained in extension for 4-7 days. They were distributed uniformly along the length of the muscle fibres. After two weeks of extension, the number of 'extra sarcomeres' was similar to that in control muscle, while the total number of sarcomeres and the length of the muscle had increased by about $30 \%$. The ultrastructure of the regions of 'extra sarcomeres' was studied electron microscopically in Epon- and melamine-embedded muscles [Bachhuber \& Frösch, J. Microsc. 130, 1 (1983)]. Special attention was devoted to irregularities of the Z-line and of the A-band. We concluded from the results that there are two mechanisms of reorganization of the contractile structure by which new sarcomeres can be formed: (1) the Z-line first increases in thickness to the size of a normal sarcomere, then it is replaced by filaments, so that one Z-sheet is divided into two Z-sheets; and (2) a new Z-line is formed within an A-band, i.e. a third $Z$-sheet appears between the two original ones.

Part of this work was performed in Section Electronenmikroskopie and Abteilung Allgemeine Physiologie, Universität Ulm, D-7900 Ulm, West Germany.

Analysis of DNA coding for the $\mathrm{Ca}^{2+}$, $\mathrm{Mg}^{2+}$-ATPase of sarcoplasmic reticulum B. Korczak ${ }^{1}$, C. Brandl ${ }^{1}$, D. H. MacLennan ${ }^{1}$ and N. M. Green ${ }^{2}$

${ }^{1}$ Banting and Best Department of Medical

Research, University of Toronto, Toronto, Canada

M5G IL6

${ }^{2}$ National Institute for Medical Research, The Ridgeway, Mill Hill, London, NW7 1AA, U.K.

The $\mathrm{Ca}^{2+}, \mathrm{Mg}^{2+}$-ATPase is the major intrinsic protein in the sarcoplasmic reticulum membrane. This enzyme is responsible for the regulation of $\mathrm{Ca}^{2+}$ concentration in the myoplasm and for the initiation of muscle relaxation. The enzyme translocates $\mathrm{Ca}^{2+}$ in a reaction that involves the transient formation of an acylphosphoprotein intermediate from ATP. The reaction cycle must be placed in context with structural features of the protein and these have been elucidated by chemical, biosynthetic and molecular biological analyses.

In our recent studies we have used recombinant DNA technology to obtain information on the structure of the gene coding for the $\mathrm{Ca}^{2+}$, $\mathrm{Mg}^{2+}$-ATPase and on the amino-acid sequence of 
this protein. A genomic library from rabbit liver DNA was screened using a recombination assay. We isolated a $17 \mathrm{~kb}$ genomic fragment which has been shown to contain the $\mathrm{COOH}$-terminus of the ATPase and which extends very close to, if not to, the $-\mathrm{NH}_{2}$ terminus. Nucleotide sequence analysis of the -COOH terminus of the ATPase gene has shown that this part is rich in short introns and exons. Introns that we have analysed have had lengths of 87,127 and 97 bp and flank exons of 138,117 and $118 \mathrm{bp}$, respectively. In this region, introns separate exons coding for hydrophobic polypeptides from exons coding for hydrophilic polypeptides.

The sequence of cDNA coding for the $-\mathrm{COOH}$ terminus of the enzyme has allowed us to construct a model for the folding pattern of the $\mathrm{Ca}^{2+}$, $\mathrm{Mg}^{2+}$-ATPase polypeptide. A sequence of some 435 amino acids that contains the site of phosphorylation and of FITC binding is exposed on the cytoplasmic side of the membrane. The molecule then passes through the membrane in a loop leaving a sequence of four amino acids in the luminal space. The sequence alternates between hydrophobic and hydrophilic stretches that suggest a sequence of 24 cytoplasmic amino acids, a single transmembrane passage, a luminal loop of 68 amino acids, a transmembrane passage and $-\mathrm{COOH}$ terminus sequence (probably cytoplasmic) of 50 amino acids.

\section{Immunological identification and sequences of the nematode sarcomeric myosin heavy chain gene family \\ D. M. Miller and J. Karn \\ MRC Laboratory of Molecular Biology, \\ Cambridge CB2 2QH, U.K.}

Four different myosin heavy chain (MHC) isoforms are produced in the nematode, Caenorhabditis elegans: myosins $A$ and $B$ are expressed in the body wall muscle cells; myosins $C$ and D are expressed in the pharyngeal musculature. The complete nucleotide sequence of the MHC B (unc 54) gene has been determined. Three additional $\mathrm{MHC}$ genes (I, II, III) which hybridize to MHC B nucleotide probes have been isolated [Karn et al, Proc. natn. Acad. Sci. U.S.A. 80, 4253 (1983)]. We have used an immunological approach to identify the myosin heavy chain isoforms encoded by these genes. Hind III fragments encompassing most of the MHC rod sequences for each gene were cloned into the plasmid pUR288 and expressed in bacteria. Novel high molecular weight proteins corresponding to $\beta$-galactosidase-MHC fusion peptides were tested for reaction with specific monoclonal antibodies by immunoblotting. Antibody $5-6$ is specific for the S2 subfragment of myosin $A$ and reacts with a fusion peptide encoded by a 460 bp Hind III fragment from the hinge region of MHC III. Similar experiments indicate that $\mathrm{MHC}$ II encodes myosin $\mathrm{C}$ and that MHC I encodes myosin D. Antibody decoration experiments have suggested that myosin $A$ rods form antiparallel and parallel oriented complexes in nematode thick filaments whereas myosin B rods are restricted to a parallel-packed orientation [Miller et al., Cell 34, 477 (1983)]. We are currently completing the nucleotide sequence of the $\mathrm{MHC}$ III gene in order to examine this phenomenon through model building studies [McLachlan \& Karn, J. molec. Biol. 164, 605 (1983)].

D.M.M. was supported by grants from the Burroughs-Wellcome Foundation and the European Molecular Biology Organization.

\section{Structure of the human muscle tropomyosin gene family \\ Girish Modii ${ }^{1}$, Frank S. Walsh ${ }^{1}$, Kathleen Talbot ${ }^{2}$ \\ and Alexander R. Macleod ${ }^{2}$ \\ ${ }^{1}$ Molecular Neurobiology Laboratory, Institute of \\ Neurology, London, U.K. \\ ${ }^{2}$ Ludwig Institute for Cancer Research, M.R.C. \\ Centre, Cambridge, U.K.}

The analysis of skeletal muscle contractile protein gene families serves as model systems for the study of tissue specific gene expression and developmental regulation. We are studying the expression and control of the tropomyosin (TM) gene family in human skeletal muscle.

Human $\alpha$ and $\beta$ TM clones were isolated from a human skeletal muscle cDNA library cloned in the Okayama and Berg vector and donated by $\mathrm{L}$. Kedes [Gunning et al., Molec. cell. Biol. 3, 787 (1983)]. Positive clones were identified by hybridization with rabbit $\alpha$ and $\beta$ TM clones corresponding to amino-acid sequences 101-I46 and 131-172 respectively [Putney et al., Nature, Lond. 302, 718 (1983)]. Three specific profiles of hybridization were found. These were fragments that reacted with the rabbit $\alpha$-TM probe, fragments that were $a, \beta$ crossreactive and fragments that were $\beta$-specific. A 1.2 Kb PVU II-Hind III fragment from a $\beta$-TM specific clone was isolated and subcloned into M13mp8 and sequenced by the Sanger procedure. Extensive amino-acid sequence homology was found with the known sequence of rabbit $\beta$-TM and the human CDNA contains sequence information from amino acid 150 to the 
polyA tail. A $0.6 \mathrm{~Kb}$ PVU II-Hind III fragment of the $a$-TM specific clone is being sequenced. Southern analysis of Hind III digested human DNA revealed five bands of $4.2,1.5,0.9,0.4$ and $0.2 \mathrm{~Kb}$ for $a$-TM and four bands of $16,4.2,1.5$ and $0.6 \mathrm{~Kb}$ for $\beta$-TM. Interestingly the 4.2 and $1.5 \mathrm{~Kb}$ bands are reactive with both probes. To attempt to analyse the structure of these genomic sequences further a Hind III genomic library of human DNA. cloned in $\lambda_{1059}$ was screened with the $\alpha$ and $\beta$-TM cDNA fragments. A number of clones have been isolated and are being analysed. Southern analysis of Hind III digested human and mouse DNA showed that different bands are detected across species suggesting that these genes may be assigned to a specific human chromosome and to determine whether the $\alpha$ and $\beta$-TM genes are linked. These studies are now in progress.

\section{Relationship between RNA methylation and differentiation in L5 myoblast line \\ C. Morganti ${ }^{1,2}$, S. Agostini ${ }^{1}$, R. Strom ${ }^{\mathrm{I}}$ \\ G. Cantoni ${ }^{3}$ and S. Scarpa ${ }^{1,2}$ \\ ${ }^{1}$ Department of Human Biopathology, University \\ of Rome 'La Sapienza' and ' Cell Biology \\ Laboratory, Istituto Superiore di Sanitá, Rome, \\ Italy \\ ${ }^{3}$ Laboratory of General and Comparative \\ Biochemistry, NIMH, Bethesda, Maryland, U.S.A.}

Several experimental data demonstrate that DNA methylation controls gene function and differentiation in animal cells. L5 myoblast cell line can be maintained in the undifferentiated state for many passages in growth medium (10\% FCS) or be induced to differentiate into multinucleated fibres in fusion medium ( $1 \%$ FCS). We used therefore this line as an ideal model to study the involvement of methylation processes during differentiation [Scarpa et al., Proc. natn. Acad. Sci. U.S.A. 81, 3064-8 (1984)]. We thought to investigate the possible changes in RNA methylation levels, in fusing and nonfusing conditions, by adding 3-deazaadenosine (DZA), a potent methylation inhibitor [Chiang et al., Molec. Pharmacol. 13, 939-47 (1977)], and Concanavalin-A (Con-A), which increases RNA methylation in T-lymphocytes [Grünert \& Schäfer, Expl Cell Res. 140, 137-47 (1982)]. Cells were treated with DZA or Con- $A$ the day after plating and maintained in the drugs throughout the duration of the culture. De novo synthesis of purines was inhibited by adding adenosine and guanosine; the alternative methylation way through tetrahydrofolate was inhibited by formiate. The cells were labelled with $\left[{ }^{3} \mathrm{H}\right] \mathrm{CH}_{3}$-methionine and $\left[{ }^{14} \mathrm{Cluridine}\right.$ and total RNA was purified with phenol and chloroform/ isoamil alcohol. The results obtained in growth medium demonstrate that increasing or decreasing the extent of RNA methylation does not induce per se the differentiation of L 5 myoblast. On the other hand, in fusion medium the RNA methylation levels appear to be in inverse relationship with the induction of the phenomenon. RNA rate of synthesis decreases as well, but not as markedly, with culture ageing.

\section{Identification of a human fetal form of $\mathrm{LC}-2$ type myosin light chain \\ F. Pons and'J. J. Léger \\ LPM2, INSERM U 249, Institut de Biologie, \\ Bd Henri IV, 34000 Montpellier, France}

It has been shown that embryonic muscle tissues from rats and humans contained a myosin light chain of the LCI type, called $\mathrm{LCI}_{\text {emb }}$ [Whalen et al., J. molec. Biol. 126, 415-31 (1978); Strohman et al., Science 221, 955-7 (1983)]. On classic two-' dimensional polyacrylamide gel electrophoresis, this additional fetal protein differently migrated from the various adult forms of skeletal muscle myosin light chains, these last being apparently present at high levels in the fetal tissues. Using a different two-dimensional polyacrylamide gel analysis involving isoelectrofocusing for the first dimension but urea instead of sodium dodecyl sulphate for the second dimension (Pons et al, Muscle Nerve 6, 40-7 (1983)], we have observed that human fetal muscles contained a main myosin light chain of the LC2-type which differently migrated from the various adult forms of skeletal muscle myosin light chains. This human fetal LC2-type myosin light chain was practically absent in normal adult human muscle, copurified with myosin and was in vitro phosphorylable or dephosphorylable. This typical fetal LC2-type protein has been electrophoretically observed in skeletal muscle biopsies from patients with Steinert muscle diseases.

These results suggest that human fetal muscle at previous stages of development could contain only fetal specific LC2-type myosin light chains without the simultaneous presence of any adult specific LC2-type myosin light chain. This fetal protein could be a marker for studies of specific human muscle diseases, involving transitions from true adult to embryonic or fetal phenotypes. 


\section{Macromolecular methylation and its effect on protein synthesis in differentiating myoblasts of the line L5}

\author{
A. Sala ${ }^{1,2}$, R. Strom ${ }^{1}$, G. Canton ${ }^{3}$ and S. Scarpa ${ }^{1,2}$ \\ ${ }^{1}$ Department of Human Biopathology, University \\ of Rome 'La Sapienza' and ' Cell Biology \\ Laboratory, Istituto Superiore di Sanitá, Rome, \\ Italy \\ ${ }^{3}$ Laboratory of General and Comparative \\ Biochemistry, NIMH, Bethesda, Maryland, U.S.A.
}

The role of methylation on the regulation of gene expression has been suggested in several recent reports. This hypothesis is supported by a number of examples that demonstrate an inverse relationship between the extent of methylation of specific genes and their transcriptional activity. We have studied, on the myoblast cell line L5, under proliferative conditions and at different stages of differentiation and fusion, the effects of 3-deazaadenosine and Concanavalin $A$, which respectively inhibit and stimulate methylation at macromolecular level, showing a possible connection between differentiation and RNA methylation. Using the same model, we investigated whether 3-deazaadenosine and Concanavalin A, through their ability to interfere with methylation, could affect, besides fusion, also synthesis and accumulation of specific proteins. The cells were labelled with methionine ${ }^{35} \mathrm{~S}$ and the proteins were analysed by two-dimensional gel electrophoresis followed by autoradiography. For this research we selected two clones off the L5 line: clone $3 \mathrm{C} 4$, which normally fuses around $70 \%$, this process being totally inhibited by $4 \mu \mathrm{g} \mathrm{ml}^{-1}$ Concanavalin A; clone $3 \mathrm{~B} 5$, which spontaneously fuses less than $10 \%$, and reaches over $50 \%$ fusion upon addition of $5 \mu \mathrm{M}$ 3-deazaadenosine plus $100 \mu \mathrm{M}$ homocysteine. The electrophoretic pattern of the proteins obtained from clone $3 \mathrm{C} 4$, treated with Concanavalin A, closely resembles that of the proliferating cells (grown in 10\% FCS), with no fusion in either case, while the controls in I\% FCS, with a high percentage of fusion, show sharp differences. The same kind of experiment, performed with clone 3B5, but treating the cells in 1\% FCS with 3-deazaadenosine and homocysteine, gives highly different protein electrophoretic patterns of the cells treated with the drugs and extensively fused, with respect to those of the control cells, the latter being very similar to that of cells replicating in $10 \% \mathrm{FCS}$. The protein patterns of the two clones are similar under similar conditions of differentiation.
Lateral white muscle of the eel at different growth stages

P. A. Scapolo ${ }^{1}$, G. Romanello ${ }^{2}$, S. Luprano ${ }^{1}$ and

F. Mascarello ${ }^{2}$

Istituto di Anatomia degli Animali Domestici,

${ }^{1}$ Università di Bologna and ${ }^{2}$ Milano, Italy

In black eels, $25-50 \mathrm{~cm}$ in length, the white muscle is composed of a mosaic of fibres of widely differing diameters. The large fibres have an alkali-stable myosin ATPase activity and react with polyclonal antisera specific for fish white muscle myosin. Whereas most intermediate and some small diameter fibres have the same immunohistochemi$\mathrm{cal}$ and histochemical profile as the large white fibres, many of the smallest fibres also react with antisera specific for fish red muscle myosin (anti-R). However, this reaction with anti- $R$ is probably a crossreaction, because it occurs only at concentrations higher' than the minimum necessary to stain the red muscle. Two-dimensional gel electrophoresis of single fibres has shown that all the small white fibres examined contained the same fast-type myosin light chains as in the large white fibres. Ultrastructural characteristics of small white fibres are also the same as in the large white fibres, e.g. well-packed myofibrils, few ribosomes etc.

In contrast, in glass eels of only $6-8 \mathrm{~cm}$ length there are no very small fibres in the white muscle, which consists only of fibres of similar diameter and immunohistochemical profile, equivalent to the large white fibres of the older eels.

The appearance of small white fibres in the older eels is thought to be related to growth, but raises the question of their origin. An electron microscopical study of the white muscle of the glass eels (which do not yet have the very small white fibres) has revealed the presence of myosatellite cells lying under the basal lamina of the muscle fibres. Presumably the very small fibres appearing in later stages (such as the black eel) arise from these myosatellite cells, and this is supported by the immunohistochemical evidence, as developing fibres are likely to pass through a stage in which they contain some form of 'embryonic' myosin unlike that of the adult fast white fibres.

This work was supported by the M.P.I. (40\%).
Differentiation of fibre types in rat skeletal muscle visualized with monoclonal antimyosin antibodies
S. Schiaffino, L. Saggin, A. Viel and L. Gorza
Institute of General Pathology, University of
Padova, 35100 Padova, Italy
Monoclonal antibodies (mAbs) generated from 
mice immunized with bovine fetal myosin and reactive with myosin heavy chains (MHCs) were used to study isomyosin transitions during postnatal development in rat skeletal muscle fibres. Most mAbs showed complex patterns of crossreactivity between different families of MHCs. A number of $\mathrm{mAbs}$ reacted specifically with adulttype MHCs which were absent in fetal muscle and were found to accumulate during postnatal development. Others mAbs were selectively reactive with fetal-type MHCs that were progressively lost after birth. Developmental changes in the pattern of immunoreactivity were fibre-type specific and were found to vary between different mAbs. Two fibre types, destined to become the type $I$ and type 2 fibre populations in adult muscle, were clearly distinguished by different $m A$ bs since fetal stages in the fast tibialis anterior and extensor digitorum longus muscles. The type 2 fibre population was apparently homogeneous in neonatal muscle with respect to antimyosin immunoreactivity. Diversification of type 2 fibres was first observed during the first postnatal week but was not completed until the fourth week. Three type 2 fibre subpopulations with distinct pattern of immunoreactivity, suggesting the existence of at least three subsets of type $2 \mathrm{MHCs}$, were identified in mature fast muscles.

Supported by grants from CNR and Ministero Pubblica Istruzione.

\section{Messenger RNA complexity and expression of skeletal and cardiac actin genes during rat cardiac hypertrophy}

K. Schwartz, J. M. Moalic, D. de la Bastie,

C. Wisnewsky, P. Bouveret, J. Bercovici and

B. Swynghedauw

INSERM U 127, Hôpital Lariboisière, 41 Bd de la

Chapelle, 75010 Paris, France

Chronic haemodynamic overload stimulates the synthesis of most of the cardiac proteins. In order to study the overall switch in gene expression, the kinetic of hybridization of cardiac $\mathrm{mRNA}$ and of its corresponding $\mathrm{CDNA}$ has been studied $\left(R_{0} t\right)$. Computerization of results in normal heart showed that the best fit of the data was a set $(N=1,2,3)$ of three pseudo-first order components corresponding to three abundancy classes containing respectively 8,790 and 40200 different sequences which had been copied 45000,500 and 8 times respectively. Four days after aortic stenosis the number of copies was increased by $30 \%$ in every class. One month after a stenosis the kinetic of hybridization was unchanged. To analyse the gene sequences expressed during this type of cardiac growth more precisely, we examined the mRNAs coding for skeletal and cardiac actins. The cDNA plasmids, constructed from mouse skeletal muscle, were generously supplied to us by S. Alonso and $\mathrm{M}$. Buckingham [Minty et al., Cell 30, 185 (1982)]. It was found that only trace amounts of adult skeletal muscle actin mRNA are expressed in normal and sham-operated animals. Two to eight days after aortic banding we observed a 2-16-fold increase in the amount of this mRNA, which decreased again after 15 days of stenosis. It is impossible as yet to assess the biological significance of this coexpression of skeletal and cardiac actin genes at the onset of cardiac hypertrophy. Our results however indicate that the time course of the actin isoform switches does not parallel that of myosin heavychains $a$ and $\beta$ [Mercadier et al., Circ. Res. 48, 525 (1981)]. This strongly suggests that the differential expressions of the two multigene families, myosin and actin, do not play identical roles in cardiac hypertrophy.

\section{Effects of ontogenic development, thyroid hormone and haemodynamic overload on rat atrial isomyosins}

K. Schwartz, J. L. Samuel, I. Syrovy, F. Marotte, C. Wisnewsky and L. Rappaport

INSERM U 127, Hôpital Lariboisière, 41 Bd de la Chapelle, 75010 Paris, France

The expression of myosin heavy chain isoforms $\mathrm{HC} a$ and $\mathrm{HC} \beta$ in mammalian cardiac ventricles varies with age and is altered as a result of thyroid hormone imbalances or increased haemodynamic load [reviewed in Samuel et al. Circ. Res. 52, 200 (1983)]. Recent studies have shown that atrial myosins are also composed of two isoforms, one of which is indistinguishable from ventricular $\mathrm{HC} \alpha$, but that, contrarily to ventricular tissue, changes in the thyroid hormone levels or in the ventricular load do not markedly modify their pattern. To determine if indeed atrial tissue does not respond to the above stimuli, we used sensitive immunocytochemical procedures and antibodies specific of ventricular $\mathrm{HC} a$ and $\mathrm{HC}_{\beta}^{*}$ with the aim to detect on atrial cryostat sections small amounts of each isomyosin. We observed that in rats, whatever the developmental stage (fetus, young or adult) all atrial cells were stained with anti-HCa. In contrast, the pattern of staining with anti-HC $\beta$ was developmentally regulated: fetal tissue contained

${ }^{*}$ Anti-HC $\beta$ immunoglobulins were a gift from S. Sartore and S. Schiaffino, Padova, Italy. 
numerous labelled fibres, which almost completely disappeared soon after birth. Low iodine diet during pregnancy and lactation inhibited the postnatal regression of $\mathrm{HC} \beta$, but had no effect on the number of cells stained by anti-HC $\alpha$. Daily injection of thyroxine to 10-day-old hypothyroid rats caused a rapid decrease in $\mathrm{HC} \beta$ labelling. In adults, hypophysectomy did not influence $\mathrm{HC} a$ labelling, but markedly increased that of $\mathrm{HC} \beta$; this latter effect was completely reversed by thyroxine administration. Gross haemodynamic overload of the left ventricle (aortic banding, 150\% hypertrophy) induced also an increase of $\mathrm{HC} \beta$-containing cells. We conclude that the expression of atrial myosin isoforms is regulated by the same stimuli as the ventricular tissue, but that the response is quantitatively very different.

\section{Patterns of actin isoforms expression in fibroblastic and smooth muscle tissues in vivo} O. Skalli ${ }^{1}$, G. Gabbiani ${ }^{1}$, J. Vandekerckhove ${ }^{2}$ and K. Weber ${ }^{3}$

${ }^{1}$ Department of Pathology, University of Geneva, 1211 Geneva 4, Switzerland

${ }^{2}$ Laboratory of Histology and Genetics, Ledeganckstraat 35,9000 Ghent, Belgium

${ }_{3}^{3}$ Max-Planck Institute for Biophysical Chemistry, 3400 Goettingen, Federal Republic of Germany.

The pattern of actin isoforms was examined in different fibroblastic and smooth muscle tissues of human and nonhuman origin. Two methods were used: planimetric analysis of silver stained bidimensional gels [Quitschke \& Schechter, Analyt. Biochem. 24, 23I-8 (1982)] and migration of actin$\mathrm{NH}_{2}$ terminal peptides on bidimensional cellulose thin layer electrophoresis [Vandekerckhove \& Weber, Eur. J. Biochern. 113, 595-603 (1981)]. Fibroblastic tissues such as derm and tendon were always found to contain only $\beta$ - and $\gamma$-cytoplasmic isoforms, $\beta$ being predominant over $\gamma$; whereas smooth muscle tissues contained $a_{-}, \beta$ - and $\gamma$ isoforms, but the proportions of isoforms were different in different muscles. Thus, the presence or absence of the $a$-isoforms of actin represents a reliable criterion for the distinction between fibroblastic and smooth muscle cells and/or tissues; this distinction may be useful in the study of differentiation and of pathological phenomena, such as wound healing or atheromatosis, and for the determination of the origin of certain soft tissue tumours. Furthermore, the heterogeneity of actin isoform pattern, especially in smooth muscles, suggests that the differential expression of actin isoforms is related to specific cellular or tissue functions.

(Supported by the Swiss National Science Foundation, Grant Nr 3.178-.0.82).

\section{Developmental and tissue specific regulation of a repetitive element present in an $\mathrm{L} 6$ myotube specific cDNA clone}

A. Starzinski-Powitz and T. Herget

Institute of Genetics, University of Cologne, Weyertal 121, 5000 Cologne 41, Federal Republic

of Germany

We have isolated a cDNA clone, pL6-411, from a L6 myotube cDNA library which contains a 1500 $\mathrm{bp}$ insert. The insert sequence or part of it is reiterated and interspersed in the rat genome as demonstrated by Southern blot analysis.

The expression of the repetitive element present in pL6-4II is developmentally regulated during myogenesis in culture. A set of transcripts can be identified by Northern blot analysis with pL6-4II in the poly $\mathrm{A}^{+}$-enriched cytoplasmic RNA fraction of L6 cells $24-36 \mathrm{~h}$ after induction of myotube formation. Northern blot analysis with RNA isolated from fetal and adult skeletal muscle shows that only a set of poly $\mathrm{A}^{+}$transcripts from fetal muscle hybridizes to pL6-411. Since at least most of the transcripts containing the pL6-41I repetitive element appear to accumulate in a coordinated fashion during myogenesis in vitro and in vivo we suggest an important regulatory role for this repetitive element. Additional Northern blot analysis of RNAs isolated from various rat tissues showed that: (I) brain, thymus and spleen each contain prominent quantities of the transcripts expressing the pL6-4II repetitive element. (2) Rat heart transcripts hybridize also to pL6-4II but the number of transcripts containing the repetitive element appears to be lower than in the tissues mentioned above. (3) Kidney RNA shows only a very faint hybridization to PL6-41I which could be due to the neuronal cell content in the kidney tissue. (4) The pL6-4II repetitive element does not appear to be expressed in liver. These data suggest that the pL6-411 repetitive element is also regulated in a tissue specific fashion. Subcloning of pL6-41I and DNA sequence analysis is in progress. 


\section{Cardiac and skeletal muscle actin in adult pig heart, fetal skeletal muscle and L6 myotubes: a quantitative determination of both isoforms}

J. Vandekerckhove ${ }^{1}$, G. Bugaisky ${ }^{2}$ and

M. Buckingham ${ }^{2}$

Laboratory of Genetics, State University Ghent,

Belgium

${ }^{2}$ Laboratory of Molecular Biology, Institut Pasteur,

Paris, France

Recent literature provides growing evidence that both cardiac and skeletal muscle actin mRNAs are present in striated muscle tissue of various origin. Although protein-chemical data revealed that cardiac actin was the major isoform in adult heart tissue and skeletal muscle actin the major isoform in fast and slow skeletal muscle tissue, it was interesting to know, especially in view of the observed mRNA levels, to what extent these actin isoforms were also expressed as minor forms in other tissues.

So far, both actin isoforms could not be separated from each other since they only differ by four conserved amino-acid substitutions and show identical molecular and functional properties.

Here we provide a method for quantitative determination of each of the two striated muscle isoactins in the presence of the other actin isoforms. (1) The mixture of the $-\mathrm{NH}_{2}$ terminal tryptic peptides of striated muscle actins is isolated; (2) these peptides are cleaved with the $\mathrm{V} 8$ protease and the mixture of the two $-\mathrm{NH}_{2}$ terminal tetrapeptides isolated (Acetyl-Asp-Glu-Asp-Glu for skeletal muscle and Acetyl-Asp-Asp-Glu-Glu for cardiac actin); (3) the tetrapeptide mixture is partially hydrolysed and the tripeptide mixture is isolated (Asp-Glu-Asp and Glu-Asp-Glu for skeletal muscle actin and Asp-Asp-Glu and AspGlu-Glu for cardiac actin); (4) tripeptides are $\mathrm{NH}_{2}$-terminally blocked and their Glu/Asp ratios compared with the Glu/Asp ratios of similarly prepared reference mixtures containing varying ratios of cardiac and skeletal muscle actin ( $100 \%$ cardiac actin gives a Glu/Asp $\simeq 7 ; 100 \%$ skeletal muscle actin gives a Glu/Asp = I); (5) the amounts of cardiac and skeletal muscle actin in the reference mixtures are determined by comparing the Glu/Asp ratios of the tripeptides before and after blocking the $\mathrm{NH}_{2}$-terminal residue.

These analyses reveal that cardiac and skeletal muscle actin are expressed in striated muscle tissue in ratios which are in good agreement with the estimated levels of the corresponding mRNAs: rabbit skeletal muscle: $95 \%$ skeletal, 5\% cardiac; pig heart : $20 \%$ skeletal, $80 \%$ cardiac; L6 myotubes : $75 \%$ skeletal, $25 \%$ cardiac; mouse fetal muscle : $75 \%$ skeletal, $25 \%$ cardiac.

\section{Control of myosin isozyme transitions by hormonal changes occurring during muscle development}

Robert Whalen, Madeleine Toutant, Gillian Butler-Browne, Graham Riley and Simon Watkins Département de Biologie Moléculaire, Institut Pasteur, 25 Rue du Dr Roux, 75724 Paris, France Of the ten myosin heavy chain polypeptides found in vertebrate animals, four principal isozymes (embryonic, neonatal, slow and adult fast) are found in developing skeletal muscles. It is possible to explain the isozyme transitions observed in developing rat muscles by considering that one sequence of transitions is: embryonic-neonataladult fast. Superimposed on this sequence, the induction of slow myosin can occur at any time in development.

The transition neonatal--adult fast can take place in the absence of innervation in young rats. However, chemically induced hypothyroidism leads to an inhibition of the appearance of adult fast myosin. To investigate further the endocrinological factors controlling this transition, we have examined muscle of mutant mice manifesting a syndrome of hereditary pituitary dwarfism. In these animals the myosin isozyme transitions of skeletal and cardiac muscles are affected. These two tissues differ, however, in that the transition in skeletal muscle takes place more slowly than in normal mice while the cardiac transition is completely inhibited up to at least 75 days after birth. Injection of thyroid hormone $\mathrm{T} 4$ induces accumulation of the adult isozymes and leads to disappearance of the developmental forms in both tissues within 11-12 days. The characteristics of the dwarf mutation suggest the conclusion that lack of thyroid hormone rather than growth hormone is responsible for the alteration of myosin isozyme transitions. This mouse mutant should be a useful model for further study of the control of myosin isozyme transitions.

\section{Developmental aspects of the effects of muscle denervation in birds and mammals \\ C. Wittenberger \\ Biological Research Centre, Str. Republicii 48, \\ R-3400 Cluj-Napoca, Romania}

Experiments were conducted on chickens and young rats, in order to elucidate some age- and species-specific features of the neuro-humoral 
regulation of muscle metabolism. Three age groups were chosen in both chickens and rats, starting with the age when independent nutrition begins. Comparative developmental aspects of the effects of muscle denervation are summarized here. Several indexes were coined and their values calculated, in order to allow numerical expressions of the ontogenetical trends, and interspecies comparisons. The relative ontogenetical index, expressing the amplitude of denervation-elicited modifications as related to those at age I, gave for ages II and III the mean values 4.4 and 1.8 in chickens, 0.9 and 0.6 in rats; the index of recovery, expressing the tendency of denervation-elicited metabolic disturbances to develop, always gave negative values in rats (i.e. no recovery of muscle metabolism in the preatrophic stage), but positive ones in chickens age II; the index of reversed ontogenesis, expressing the degree to which denervation counteracted the normal developmental trends, gave for the three ages the values: 28,68 , and 157 in rats, 20,109 , and 68 in chickens; the 'denervation versus hormonal treatments' indexes gave values generally increasing with age, in both species.

Conclusions: (1) Denervation-elicited modifications of muscle metabolism are strongly agedependent; (2) the normal development of muscle metabolism is more strongly dependent on neural regulation in the mammal (rat) than in the bird (chicken). (The experimental data on which the calculus of the above indexes is based were obtained in collaboration with D. Coprean, Rodica Giurges and G. Frecus, from the same laboratory.)

\section{MYOFIBRILLAR STRUCTURE, MYOFIBRILLAR PROTEINS, MYOSIN AND ACTIN}

\section{Fluorescence studies on intracellular dansylaziridine labelled troponin- $\mathrm{C}$ in single Balanus nubilus muscle fibres C. C. Ashley ${ }^{1}$, P. J. Griffiths ${ }^{1}$, P. Strang ${ }^{2}$ and J. D. Potter ${ }^{2}$ \\ ${ }^{1}$ University Laboratory of Physiology, South Parks Rd, Oxford OXI 3PT, U.K. \\ ${ }^{2}$ Department of Pharmacology, University of Miami, Florida, U.S.A.}

The calcium binding subunit of troponin $(\mathrm{TnC})$ can be labelled with dansylaziridine to produce a fluorescent species $\left(\mathrm{TnC}_{\mathrm{DANZ}}\right)$ in which fluorescence increases when either of the two calcium low affinity sites is occupied by calcium ions. It has been shown [Potter \& Zot, Biophys. J. 37, 43a (1982)] that free $\operatorname{TnC}_{\mathrm{DANZ}}$ and the whole troponin moiety on the actin filament have similar affinities for calcium ions, and hence the fluorescence of free $\mathrm{TnC}_{\mathrm{DANZ}}$ inside a cell should be an indication of the level of occupancy of both low affinity sites on the native troponin on the actin filaments. We have injected $\mathrm{TnC}_{\mathrm{DANZ}}$ into barnacle muscle fibres (final intracellular concentration $50-100 \mu \mathrm{M}$ ), and have studied changes in fibre fluorescence during stimulation under voltage clamp control. The injection of $\mathrm{TnC}_{\mathrm{DANZ}}$ into the fibre causes the appearance of a fluorescence peak at $516 \mathrm{~nm}$ on excitation at $325 \mathrm{~nm}$. This additional fluorescence signal declines on either side of the peak wavelength to disappear outside the range 450-600 nm.
A similar emission frequency spectrum for $\mathrm{TnC}_{\mathrm{DANZ}}$ in vitro has been obtained by Johnson et al. [J. biol. Chem. 253, 645I (1978)]. Fibre autofluorescence is maximal at $420 \mathrm{~nm}$, and therefore wavelengths close to this value were chosen to monitor nonspecific changes in fibre fluorescence, since at this wavelength the contribution of $\mathrm{TnC}_{\mathrm{DANZ}}$ to the fluorescence signal is negligible. After scaled subtraction of nonspecific fluorescence changes at $440 \mathrm{~nm}$ from the signal at $520 \mathrm{~nm}$ upon electrical stimulation, a net signal was recorded whose amplitude was dependent on the intensity of stimulation. The halftime of rise of this signal generally preceded the halftime to peak force by $35 \mathrm{~ms}(N=15)$, while the halftime of the fall of fluorescence was 2.24 times longer than the halftime of mechanical relaxation $(N=11)$ at $12^{\circ} \mathrm{C}$. Force and peak light were attained at about the same moment. These observations may be accounted for in part by the rapid binding of calcium ions to the free low affinity sites on $\mathrm{TnC}_{\mathrm{DANZ}}$ followed by a slower association with sites occupied by $\mathrm{Mg}^{2+}$ (about $50 \%$ at physiological concentrations of $\mathrm{Mg}^{2+}, 5 \mathrm{mM}$ ), and indicate a delay between formation of the $\mathrm{Ca}^{2+}$-troponin complex and force generation. The slow fall of fluorescence is consistent with findings from other groups suggesting that the time course of mechanical relaxation may be much faster than the rate of decline of availability of actin binding sites for 
unattached crossbridges [Huxley \& Simmons, Cold Spring Harb. Symp. quant. Biol. 37, 669 (1973); Ashley \& Lignon, J. Physiol., Lond. 318, IOP (1981)]. Preliminary experiments with fibres injected with both aequorin and $\mathrm{TnC}_{\mathrm{DANZ}}$ suggests that the aequorin light response occurs somewhat sooner than the $\mathrm{TnC}_{\mathrm{DANZ}}$ signal and before the rise of force. Supported by the M.R.C.

\section{Is tubulin a Z-disc associated protein of the sarcomere? \\ P. Bachmann and K. Kaehn \\ Lehrstuhl für Anatomie I, Ruhr-Universität, \\ Bochum, D-4630 Bochum 1, G.F.R.}

Experiments on the occurrence and distribution of tubulin during skeletal myogenesis and myofibrillogenesis are not, or only incompletely, documented. Our results reveal an intracellular sorting of tubulin containing structures during muscle cell differentiation in culture.

Myoblasts, immature and spontaneously contracting myotubes, and additional myofibrils from chick skeletal muscle were treated with a monoclonal antitubulin antibody in indirect immunofluorescence. The antigen specificity of the antibody was examined by ELISA, immunohistochemical and immunoblotting methods.

In myoblasts and in immature myotubes (without cross-striation) antitubulin stains microtubles and their organizing centres. In sevenday-old spontaneously contracting myotubes, the antitubulin antibody reveals microtubules arranged parallel to the long axis of the syncytium, or tubulin is distributed diffusely within the syncytium. In two-week-old myotubes in vitro or in myofibrils from chick, antitubulin antibody stains tubulin associated with the Z-disc. Additional experiments are necessary to clarify whether tubulin, as stated e.g. for desmin, is an anchor protein at the Z-disc. Supported by the VW-Stiftung (I/36986).

\section{A novel thick filament protein of 86000 subunit $M_{\mathrm{r}}$ is colocalized in part with C-protein}

M. Bähler, T. Wallimann and H. M. Eppenberger Institut für Zellbiologie, ETH-Hönggerberg,

\section{$\mathrm{CH}-8093$ Zürich, Switzerland}

A myofibrillar protein was purified from crude C-protein preparations of chicken pectoralis muscle. Analysis of the protein by PAGE in $\mathrm{NaDodSO}_{4}\left(M_{\mathrm{r}}=86000\right)$ and gel permeation $\left(M_{\mathrm{r}}=370000\right)$ suggest an asymmetric or nonmonomeric structure of the native protein.
Antibodies raised in rabbits against the $86000 M_{\mathrm{r}}$ polypeptide were demonstrated by doubleimmunodiffusion and immunoblots to be specific for this protein and to show no crossreactivity with myosin, $\mathrm{M}$-band proteins, $\mathrm{C}$-protein, $\mathrm{H}$ protein and $X$-protein. The antibodies were shown by indirect immunofluorescence to label the A-band specifically, with the exception of the $\mathrm{H}$-zone, of fast (pectoralis major) but not of slow (anterior latissimus dorsi) skeletal muscle nor heart muscle fibres. This corresponded with the staining of fibres by anti-fast muscle C-protein antibodies. The labelling pattern, however, seen in the electron microscope with anti-86 $000 \mathrm{M}_{\mathrm{r}}$ protein antibodies differed from that obtained with anti-C-protein antibodies. In fast twitch skeletal muscle fibres, after incubation with anti-86000 $M_{\mathrm{r}}$ protein antibodies, nine distinct stripes were revealed per half-A-band running perpendicular to the filament axes and being spaced by approximately $43 \mathrm{~nm}$. Seven out of the nine stripes coincided with the positions of anti-C-protein labelling whereas the two additional stripes in each half-A-band reach out beyond the seven stripes with the same $43 \mathrm{~nm}$ periodicity towards the M-band. As shown by immunofluorescence in myogenic cell cultures the localization of this thick filament associated protein was restricted to the myofibrils. During muscle development the protein appeared simultaneously with C-protein and myomesin. The novel thick filament protein can be considered as a specific marker for differentiated, cross-striated skeletal muscle.

\section{DNAase $I$ binding region in actin structure}

Y. Benyamin ${ }^{1}, C$. Roustan ${ }^{2}$ and M. Boyer ${ }^{1}$

${ }^{1}$ Laboratoire de Biochimie et ${ }^{2} d$ 'Ecologie des

invertébrés marins, (Ecole Pratique des Hautes

Etudes), CRBM, CNRS, BP 5051, 34033

Montpellier Cedex, France

In nonmuscle cells, actin is found together in microfilaments and monomeric states. Many actinbinding proteins are implicated in the regulation of actin polymorphism. Thus, the nonpolymerized form of actin would be stabilized by low molecular weight proteins such as profilin, DNAaseI also forms a I:I complex with G-actin preventing actin polymerization.

We have initiated a search to locate the specific binding domains of associated proteins in the primary structure of actin. In this communication, we present the results concerned with DNAaseI-actin complex formation. We have, in particular, shown that several lots of antibodies which are specific of the N- or C-terminal part of actin respectively are 
not concerned with the DNAaseI-actin complex formation. However, it is interesting to notice that the two classes of antibodies compete with each other. Thus, these results infer a spatial proximity between the $\mathrm{N}$ - and $\mathrm{C}$-extremity of actin.

In contrast, DNAasel binding is affected by another antibody population which is specific of a central region in actin. Furthermore $\mathrm{Mg}^{2+}$ transition and actin polymerization are concerned with this class of antibodies. Finally, all these observations underline the functional importance of the central part of actin.

\section{Myosin light chains in human muscle diseases \\ R. Betto and G. Salviati \\ N.R.C. Unit for Muscle Biology and \\ Physiopathology, Institute of General Pathology, \\ University of Padova, Italy.}

By histochemical myosin ATPase reaction, four different types of fibres are detected in human skeletal muscles: type 1 , type $2 \mathrm{~A}$, type $2 \mathrm{~B}$, and type $2 \mathrm{C}$. The diverse histochemical reactivities have been correlated with the presence of different isoforms of myosin in different muscle cells. Myosin is a heteropolymeric molecule consisting of a pair of heavy chains and two pairs of light chains. Both heavy and light chains show specific isoforms in fast and slow muscles. Myosin isolated from normal human muscles demonstrates both fast and slow myosin isoforms in a proportion that roughly matches that of histochemical type 1 and type 2 fibres. In pathological muscles this correlation is frequently lost. In this study we have analysed the pattern of myosin heavy and light chains in single muscle fibres from several human myopathies (Duchenne muscular dystrophy, myotonic muscular dystrophy, dysthyroid myopathy, polymiositis). All these diseases showed as a common feature an increase in the population of intermediate fibres showing coexistence of fast and slow myosin isoforms (intermediate fibres). Based on the myosin light chain pattern two populations of fibres were found, one showing the full complement of fast and slow light chains and the other showing a set of light chains of one type and an extra chain of the other type. The analysis of the corresponding heavy chains demonstrates that many intermediate fibres contained both fast and slow myosin heavy chains. However several intermediate fibres showed only one type of heavy chain indicating that they contain molecular hybrids of myosin. Since the catalytic properties of myosin are dependent exclusively from the type of heavy chains the discrepancy between the light chain pattern of purified myosin and the histochemical composition in pathological muscles can be explained by the increased amount of molecular hybrids of myosin. The results also suggest that type $2 \mathrm{C}$ fibres are not necessarily immature fibres containing fetal myosin, but mature fibres undergoing transition from fast to slow and vice versa under the prevailing conditions in vivo. Supported by institutional funds from the Consiglio Nazionale delle Ricerche and in part by a grant from the Legato Ferrari to Professor A. Margreth.

Location of the A1 light chain in the acto-S1 complex: A fluorescence energy transfer and ${ }^{1}$ H-n.m.r. study

D. G. Bhandari ${ }^{1}$, H. R. Trayer ${ }^{1}$, B. A. Levine ${ }^{2}$ and I. P. Trayer ${ }^{1}$

${ }^{1}$ Department of Biochemistry, University of Birmingham, Birmingham B15 2TT, U.K. ${ }^{2}$ Inorganic Chemistry Laboratory, University of Oxford, Oxford OX1 3QR, U.K.

The A1 light chain of rabbit skeletal myosin has been characterized by previous studies showing that both its N-terminal and C-terminal regions are exposed to solvent when located in SI(A1). ${ }^{1}$ H-n.m.r. spectroscopy has shown that the N-terminal 41 residue portion displays high segmental mobility that becomes immobilized upon interaction with actin. On the other hand, collision quenching studies have shown that exposure of a fluorophore located at cys-178 of the AI light chain in S1(AI) is not affected by actin binding. Here, we report fluorescence energy transfer studies that were carried out between the C-terminus of the AI light chain and actin. The thiol group at cys-178 was specifically labelled with a donor fluorophore (BrAEDANS) in the isolated A1 light chain and then reintroduced into SI by published hybridization procedures. Cys-373 of actin was labelled with an acceptor fluorophore (IAF). The efficiency of transfer, $E$, was determined by measuring the quenching of donor emission. In the binary acto-SI(A1) complex, $E$ was found to be $24( \pm 1.1) \%$ under varying conditions, and corresponded to a spatial separation $\left(R^{2} / 3\right)$ of $5.7 \mathrm{~nm}$. This distance did not significantly alter when the complex was formed with fully regulated actin ( $\pm \mathrm{Ca}$ ). Formation of ternary complexes with either MgADP or MgAMP.PNP produced little change; $E=38 \%$ and $R^{2} / 3=5.1 \mathrm{~nm}$ in both cases. These results are consistent both with earlier fluorescence energy transfer studies involving the fluorescently labelled SH-I thiol group of the S1 heavy chain and cys-373 of actin and 
${ }^{3}$ H-n.m.r. results. These data and those reported above show no evidence of a large-scale conformational change in the 'rigor' binary complex when it binds either Mg.ADP or Mg.AMP.PNP.

${ }^{1}$ H-n.m.r. studies have also shown that the $\mathrm{N}$-terminal amino acid of the A1 light chain, $a-N$-trimethylalanine (Tma) is in close proximity $(<1.2 \mathrm{~nm})$ to a nitroxide spin label sited on cys-373 of actin. The enhanced relaxation induced by this paramagnetic centre is felt by the Al-Tma in SI(A1) and HMM when in complex with spinlabelled actin. Thus, the N-terminal region is separated from the C-terminal region of the A1 light chain by $4.8-7.2 \mathrm{~nm}$, and it is possible that $\mathrm{Al}$ may span more than one domain of the $\mathrm{SI}(\mathrm{AI})$ molecule. (Supported by grants from SERC and MRC.)

\section{Human ventricles contain at least three molecular variants of myosin}

P. Bouvagnet, J. Léger, C. Dechesne and J. J. Léger LPM2, INSERM U 249, Institut de Biologie,

Bd Henri IV, 34000 Montpellier, France

Successive generations of monoclonal antibodies (Mab) directed to myosin heavy chains were selected from mice immunized with different myosin preparations from human ventricular and atrial myocardium. The first generation of $\mathrm{Mab}$ was obtained from myosin prepared from the enlarged left ventricle of a 53-year-old female with an obstructive cardiomyopathy. Some of the Mab reacted in a heterogeneous way on cryostat transverse sections of fibres from the human atrial and ventricular myocardium, and crossreacted only with the ventricular V3-type myosins from hypothyroid rats. These experiments suggested the presence of two different V3-type forms of myosin within the human ventricles.

The second generation of Mab was obtained from one molecular variant of human atrial myosins prepared through immunoaffinity chromatography performed with one anti-ventricular myosin $\mathrm{Mab}$ of the first generation (see $\mathrm{C}$. Dechesne et al., Human atrium contains at least two different molecular variants of myosin). Some of these anti-atrial myosin Mab also reacted in a heterogeneous way on cryostat sections of fibres from the human ventricular myocardium but with different ventricular fibres from those previously reacting with the Mab of the first generation; the anti-atrial myosin Mab crossreacted only with the ventricular VI-type myosins from normal young rats. These experiments suggested therefore the presence of one VI-type form of myosin and finally the presence of at least three different molecular variants of myosin within the human ventricular myocardium.

Using the Mab discriminating the different myosin variants, an anatomical description of fibre types and myosin types within the human myocardium was made on the whole hearts of two normal young boys and of four patients. No clearcut conclusions can yet be made concerning the physiological role of the regional variations observed in the distribution of the different molecular forms of myosin.

\section{Light chain interactions in fast skeletal myosin filaments: a possible filament-mediated mechanism}

R. Cardinaud and M. Roux-Fromy

Département de Biologie, C.E.N. Saclay F-91191

Gif sur Yvette, France

A comparative study of the kinetics of myosin fragmentation by papain and trypsin at low and high ionic strength reveals striking differences in susceptibility of the various proteolytic sensitive zones between soluble myosin and filaments and also under the influence of usual physiological ligands $\left(\mathrm{Me}^{2+}\right.$ and nucleotides), all other conditions being identical. These observations are correlated to the behaviour of $N$-trimethylalanine proton n.m.r. lines in the filament and in monomeric myosin. In the presence of $\mathrm{Mg}^{2+}$ filament myosin is cleaved by papain according to two simultaneous patterns: (A) $200^{\circ} \rightarrow 177^{\circ}, 27^{\mathrm{N}}$ and (B) $200^{\circ} \rightarrow 110,90^{\circ}$. Added ATP or AMPPNP strongly inhibits pattern $A$. With monomeric myosin two simultaneous schemes are also observed: (B) $200^{\circ} \rightarrow 110,90^{\circ}$ and (c) $200^{\circ} \rightarrow 135^{\circ}$, 65. Pattern $A$ is no longer observed. Nucleotides have only a limited effect on the relative rates of these two cleavage reactions. In filament papain converts $\mathrm{LC}-2$ to a transient $14 \mathrm{kDa}\left(\mathrm{LC}-2^{\prime \prime \prime}\right)$ species; $\mathrm{Mg}^{2+}$ exerts a marked protective effect and this effect is enhanced in the presence of nucleotides (notably ATP). Trypsin converts LC-2 to a $18.5 \mathrm{kDa}\left(\mathrm{LC}-2^{\prime}\right)$ species very rapidly. Further cleavage leads to LC 2 '"', then complete degradation. This second step is strongly inhibited either in the presence of $\mathrm{Mg}^{2+}$ or when myosin is in the monomeric state. A similar conformational protective effect is observed for the LC-I $\rightarrow$ LC- $I^{\prime}$ $(25 \mathrm{kDa} \rightarrow 23 \mathrm{kDa}$ ) cleavage obtained with papain.

In monomeric myosin the $N$-trimethylalanine protons appear highly mobile. By alternate selective removal of LC-I and LC-2 N-terminus [Cardinaud, Eur. J. Biochem. 122, 527-33 (1982)] 
the measured line widths were 5 and $12( \pm 1) \mathrm{Hz}$ respectively. In filaments we observe a considerable broadening $(32 \pm 5 \mathrm{~Hz}$ ) of the 3.23 p.p.m. line although a number of residues in the rest of the molecule have kept a high degree of mobility. By alternately removing one or the other of the LC $\mathrm{N}$-termini the 3.23 p.p.m. line corresponding to the remaining $\mathrm{N}$-terminus recovers its sharpness, an indication that the basic $\mathrm{N}$-terminus in both these light chains is engaged in some kind of interaction with some part (in the shaft?) of the filament structure.

\section{M-band proteins myomesin, M-protein and MM-creatine kinase: A study of the immunological relationship within different species \\ Lisbeth Cerny, Monika Eppenberger, \\ Barbara Kay Grove, Jean-Claude Perriard and \\ Hans M. Eppenberger \\ Institut für Zellbiologie, ETH Hönggerberg, \\ CH-8093 Zürich, Switzerland.}

The crossreactivity of antibodies against chicken myomesin, M-protein and M-CK, three components of the M-band of cross-striated muscle, with muscles of other species was tested. Of three monoclonal antibodies (mAbs) against myomesin, one reacted with all vertebrate muscle tested. Out of four mAbs against $\mathrm{M}$-protein one reacted with all and another one with a number of vertebrate species tested. These immunological tests show that myomesin and $\mathrm{M}$-protein are conserved through evolution to a certain extent, which may be important for their ability to bind to the M-band.

On the other hand polyclonal antibodies against chicken muscle creatine kinase (MM-CK) as well as against BB-CK crossreact neither with native heterologous $C K$ isoproteins of the same species nor with native CKs from different species. However, substantial crossreactivity has been found with CKs in muscle and brain of all vertebrates tested (fish, amphibian, reptile, mammal), when the CKs had previously been denatured, indicating a conservation of antigenic determinants in the primary structure of the molecules. Based on aminoacid sequence analysis, which shows that $93 \%$ of the known sequences of chicken and rat M-CK are conserved [Benfield et al., Expl Biol. Med. 9, 187-94 (1983); Ordahl et al., unpublished], such crossreactivity would be expected. The lack of crossreactivity of anti-M-CK antibodies with native MM-CKs of other species indicates a lack of conserved surface epitopes or a very low antigenicity of these epitopes.

\section{Flexibility of smooth muscle myosin rod}

R. A. Cross

Irstitute of Molecular Biology, Austrian Academy

of Sciences, Billrothstrasse 11, A-5020 Salzburg,

Austria

A single region of local flexibility has been identified in myosin rod from mammalian (pig stomach) smooth muscle myosin, using electron microscopy of isolated molecules, native and SDS-denatured peptide mapping and viscometry.

Rods visualized by glycerol spraying followed by low-angle $\mathrm{Pt}$ rotary shadowing were $153 \pm 8 \mathrm{~nm}$ (S.D.) long, in good agreement with estimates of $156 \mathrm{~nm}$ by negative staining of segments of chicken gizzard myosin rods [KendrickJones et al., J molec. Biol. 59, 527-9 (I971)], and with a recent estimate of $154 \pm 0.6 \mathrm{~nm}$ (S.E.M.) for skeletal myosin rod [Stewart \& Edwards, FEBS Lett. $168,75-8$ (1984)]. Thirteen of 159 rods measured showed sharp $\left(>90^{\circ}\right)$ bends, $45 \pm 4 \mathrm{~nm}$ from one end. Raising the $\mathrm{pH}$ from 6.5 to $9.5 \mathrm{did}$ not induce free bending at this flexible site. LMM fragments of this rod were $99 \pm 10 \mathrm{~nm}$ long, and did not show sharp bends. Accordingly the limited flexibility observed was assigned to the proteolytically susceptible LMM-SF2 junction of this rod. The proteolytic susceptibility of this site is often taken to indicate flexibility. This possibility was tested using Cleveland peptide mapping. In the presence of denaturing amounts of SDS, the hinge was no longer especially susceptible, indicating that the susceptibility of the native hinge is due to more accessible structure, and not simply to a clustering of susceptible residues. The possibility that the hinge might become more flexible at higher temperatures was also tested, by deriving intrinsic viscosity versus temperature plots for LMM and rod over the range $5-45^{\circ} \mathrm{C}$. No change was detected for either fragment, suggesting that the hinge is either freely bending over the entire temperature range, or essentially stiff. The former possibility is excluded by the electron microscopic results.

\section{Myosin isoforms of murine fast-contracting muscles. Subunits analysis of adult and neonatal myosins: comparison with myosins of another mammalian (rat) and of an amphibian (P. waltii) \\ A. d'Albis, C. Janmot and J. J. Béchet \\ Laboratoire de Biologie Physicochimique, Université Paris-Sud, 91405-Orsay, France}

The synthesis of myosin adult isoforms in murine fast-contracting, lingual and dorsal axial, muscles is more precocious [d'Albis et al., Sassari (1983)] than 
in rat [Whalen et al., Nature, Lond. 292, 805-9 (1981)]; this may be related to an earlier increase in the level of plasmatic thyroid hormones in the case of the mouse. One observes three neonatal forms, progressively replaced by either three or four adult-type myosins. The fourth adult form, of lowest electrophoretic mobility in nondissociating gels, is composed of fast-type light chains; it differs from the intermediate myosin, present in the slow soleus, which contains both slow- and fast-type small subunits. It is totally absent from pure glycolytic white muscles, is typical of fast-oxidative red fibres, and may serve as a marker in studies involving specific modification of this type of fibre in a composite muscle. It is present in rat muscles as well and allows a neat distinction between the white tensor fasciae latae (>90\% II B fibres), the red masseter (almost 100\% II A fibres), and the pink extensor digitorum longus (about $60 \%$ II $A$ fibres and $40 \%$ II $B$ fibres). The seven isomyosins of the murine lingual muscles are composed of a combination of the same fast-type light chains. On the other hand, one observes differences between the heavy chains of the neonatal and the adult forms, as well as between the ATPase activities on the gels. This analysis has been extended to a fasttype muscle, the dorsal axial, of an amphibian, Pleurodeles walltii. The sequence of appearance of the isomyosins is more complex than in the mammals; besides three larval and three adult type forms, there are three bands with mobilities intermediate between the larval and the adult ones, which are present only through metamorphosis and in the young animal [d'Albis et al., Sassari (1983)]. These three groups of myosins are composed of the same fast-type light chains, but differ by their heavy chains and by their ATPase activities. As in mammalians at birth, metamorphosis in amphibians is accompanied by the synthesis of new myosin forms of higher enzymatic activities, in relation to the physiological requirements imposed by the new modes of life.

\section{Characterization of contractile proteins and of their proteolytic fragments by high performance liquid chromatography \\ L. Dalla Libera \\ Centro di Studio del C.N.R. per la Biologia e la \\ Fisiopatologia Muscolare, Via Loredan 16, 35100 \\ Padova, Italy}

Application of reversed-phase liquid chromatography (RPC) to peptide and protein separation has revolutionized peptide chemistry. Single amino-acid substitution is usually detectable in peptides of less than 20 amino acids. Since myosin light and heavy chains are currently used as a useful marker in studying both diseased and normal muscle tissue, it is interesting to apply RPC to the study of these proteins. We have followed two different approaches: by the first one the light chains were analysed as such, and their separation was mainly used on differences in hydrophobicity. Using a 330- $\AA$-pore C-18 column we were able to separate in well-resolved peaks the light chains isolated from fast, slow and mixed muscle myosins in less than $30 \mathrm{~min}$. The power of this method is further substantiated by the fact that good resolution is obtained also when crude myosin light chain preparations are analysed. By the second approach the light chains were previously digested with $S$. aureus $V 8$ protease in order to obtain small fragments to be analysed in $I h$ on a 100- $\AA$-pore C-I8 column. By this method we were able to demonstrate differences in the peptide maps of light chains characterized either by a high degree of homology (LCIF and LC3F) or by the same apparent isoelectric point and apparent molecular weight (the LC2S from mammals and birds). These latter findings substantiate the fact that comigration in two-dimensional gel electrophoresis is a necessary but not a sufficient condition to demonstrate identity of two polypeptides.

Myosin heavy chains were digested with $S$. aureus $\mathrm{V} 8$ protease and the fragments were separated on a $330-\AA$-pore C-18 column. Profiles with consistent differences were obtained from myosin heavy chains purified from several tissues. This procedure permits the nondestructive detection of peptides on as little as a $20-50 \mu \mathrm{g}$ sample.

\section{Human atrium contains at least two different molecular variants of myosin \\ C. Dechesne ${ }^{1}$, J. Léger ${ }^{1}, P_{1}$. Bouvagnet ${ }^{I}$, \\ M. Claviez and J. J. Léger ${ }^{2}$ \\ ${ }^{1}$ LPM2 INSERM U 249, Institut de Biologie, \\ Bd Henri IV, 34000 Montpellier, France \\ ${ }^{2}$ Max Planck Institute für Biochemie, D8083 \\ Martinsried, G.F.R.}

A few monoclonal antibodies (Mab) to myosin heavy chains prepared from one human ventricular myocardium reacted in a heterogeneous way on cryostat transverse sections of fibres from the human atrial myocardium, suggesting the presence of different forms of myosin within the human atrium. Some of these Mab have been used to fractionate preparations of native atrial myosins, permitting their further physico-chemical characterization. Two molecular variants of human atrial 
myosins or myosin fragments were thus separated by immunoaffinity chromatography performed with one anti-ventricular myosin Mab: the two atrial myosin forms have different heavy chains, the same light chains and similar, if not identical, in vitro ATPase activities. They each differ from myosins prepared from the human ventricular myocardium with respect to their heavy chains and their enzymatic activities. Structural differences between the two atrial myosins and between the atrial and ventricular myosins were located at different positions along the myosin heavy chains by peptide mapping and radioimmunoassays involving certain $\mathrm{Mab}$ reacting at different positions along the myosin heavy chains, as deduced from blotting and immunoelectron microscopy experiments.

\section{Effect of tropomyosin on the interaction of actin with actin-binding proteins isolated from pig platelets \\ E. der Terrossian and G. Prulière \\ Laboratoire de Biologie Physicochimique, \\ Université Paris-Sud, 91405-Orsay, France}

It has been shown that nonmuscle tropomyosins can protect filamentous actin against interaction with the other binding proteins [Fattoum et al., Biochemistry 22, 1187-93 (1983)]. In order to investigate the potential role of platelet tropomyosin in cellular motility, we have developed a method which allows the purification of ABP. $a$-actinin, vinculin, gelsolin and tropomyosin from the same batch of cells. After lysis in low salt buffer and high-speed centrifugation, ABP and $a$-actinin remained with the insoluble residue, while the three other proteins were mainly found in the cytoplasmic supernatant. $\mathrm{ABP}$ and $a$-actinin were then extracted by a procedure similar to that described by Burridge \& Feramisco [Cold Spring Harb. Symp. quant. Biol. 46, 587-97 (1982)]. The five proteins were further purified by gel filtration on G 150 Sephadex, and ionic exchange chromatography (DEAE, CM or phosphocellulose and hydroxy-apatite). Their identification was carried out either by their electrophoretic and isoelectrofocusing mobilities or by blotting. Antigelsolin and anti-vinculin were kindly provided by Dr A. Fattoum, Montpellier, and Dr D. Louvard, Paris, respectively. Gelsolin was obtained in a pure form which avoids the dissociation of its complex with actin by urea. Characterization of the proteins has been achieved by the study of their interactions with platelet actin. Electron microscopy and high or low shear viscosity experiments indi- cate that pig platelet tropomyosin protects the integrity of actin filaments against severing and crosslinking, but sedimentation assays showed that it does not completely prevent binding with the other associated proteins. Our results suggest that pig platelet tropomyosin stabilizes the actin filament but might have little or no effect on the interaction of these proteins with the ends of the filaments. A similar observation has been reported by Weber et al. [Biophys. J. 41, 297a (1983)] with villin isolated from microvilli.

\section{Diversity of M-band structure in cryosections of chicken breast muscle} A. C. Edman ${ }_{1}^{1}$ S. Kidman', J. Lexell ${ }^{1}$, M. Sjöström ${ }^{1}$ and J. M. Squire ${ }^{2}$

${ }^{1}$ Department of Anatomy and Neurology.

University of Umeå, Sioden

${ }^{2}$ Department of Physics, Imperial College, London, U.K.

Longitudinal cryosections and plastic sections of chicken pectoralis muscle have been used to study in detail the axial structure of the M-band, since almost all previous biochemical studies of M-proteins have used this muscle. In addition, large transverse cryosections including most of the muscle cross-section have been used to study the distribution of fibre properties across the muscle.

Although relatively homogeneous in fibre content compared with other vertebrate muscles according to normal histochemical criteria, there was, in fact, an obvious gradation of fibre structure and properties across the muscle. The bulk of the muscle, the white part, stained conventionally for type II fibręs according to mATPase test at $\mathrm{pH}$ 9.4, but in the small red part of the muscle there were also type I fibres together with the type II fibres. Superimposed on this division into type I and type II fibres were variations in oxidative and glycolytic staining properties, variations in thin filament length and I-band appearance and variations of Z-band width and M-band structure.

It was found that the M-band density at M4 and M4' (nomenclature of Sjöström \& Squire, 1977) is comparable in all fibres, but that the densities of MI and Mó vary systematically. Some fibres (probably fast) had MI strong, M6 weak (a three-line $\mathrm{M}$-band) and the Z-band was narrow. Other fibres, especially in the red part of the muscle and probably slow (type I) had Mo strong, M1 weak (a four-line M-band) and the Z-band was broad. However, the majority of fibres ranged in structure between those two extremes and had a more or less five-line M-band with M1 and M6 
both strong and a Z-band of intermediate width. Since it was such a constant feature, the M4 lines may be the sites of the primarily structural component of the M-band, whereas the different proteins at M1 and M6, possibly enzymes, may vary in quantity according to the physiological needs of the fibre.

A detailed analysis of the various observed $\mathrm{M}$-band appearances has revealed clear substructure within the strong M-bridge lines; MI contains four sublines of different strengths and $\mathrm{M} 4$ and M6 are each pairs of sublines with characteristic features. Whether these sublines represent additional unknown M-proteins or substructure within proteins at these positions is not yet known. What is certain is that chicken breast muscle is far from being homogeneous.

\section{Conformational changes in myosin subfragment- 1 induced by either tryptic digestion or nucleotide entrapment: an ${ }^{1}$ H-n.m.r. study Andrew J. Goodearl ${ }^{1}$, Barry A. Levine ${ }^{2}$ and Ian P. Trayer ${ }^{1}$ ${ }^{1}$ Department of Biochemistry, University of Birmingham, Birmingham B15 2TT, U.K. ${ }^{2}$ Inorganic Chemistry Laboratory, University of Oxford, Oxford OXI 3QR, U.K.}

Two structural analogues of SI have recently been described: (I) trypsinized SI $\left(\mathrm{SI}_{\mathrm{T}}\right)$, in which the SI heavy chain is cleaved in two regions to produce a $(27 \mathrm{~K}-50 \mathrm{~K}-20 \mathrm{~K})$ species which remains hydrodynamically intact, possesses a full complement of ATPase activities, except that the MgATPase is no longer actin activatable, and still binds to actin. (2) Crosslinked SI (SI $)^{\prime}$ in which MgATP is entrapped in the active site by crosslinking $\mathrm{SH}_{1}$ and $\mathrm{SH}_{2}$. This species is no longer enzymically competent but behaves as an SI.ATP analogue in binding studies. We have carried out ${ }^{1} H$-n.m.r. studies on these two SI analogues and found: (I) $S 1_{T}$. Two main effects were discernible: (a) a loss of some sharp signals from various regions of the spectra. The chemical shifts of these were consistent with their arising from the proposed 'loop' regions between the tryptic S1 domains and therefore suggest that these are indeed regions of high segmental flexibility. (b) A general increase in the number of protons 'visible' in the broad $\mathrm{CH}_{2} / \mathrm{CH}_{3}$ envelope. This implies that more immobile regions of the molecule, possibly representing the interior, are becoming relatively more mobile and hence contribute more obviously to this envelope. It may represent a loss of constraint on the SI structure originally imposed by the 'loop' regions. The addition of actin to $\mathrm{SI}_{\mathrm{T}}$ causes a reduction in the intensity of signals comprising the broad $\mathrm{CH}_{3} / \mathrm{CH}_{2}$ envelope in the same way as was noted for SI earlier. This suggests a reduction in internal mobility is occurring with both species. Small differences, however, are evident between the two acto-S1 complexes. (2) $S 1(A 1)_{\chi}$. Crosslinking the $\mathrm{SH}_{1}$ and $\mathrm{SH}_{2}$ groups of $\mathrm{SI}(\mathrm{A} 1)$ caused a general reduction in the mobility of the N-terminal region of the AI light chain; a region that shows high segmental mobility in S1(A1). Actin binding to SI(A1) $\times$ was indicated by specific effects on some of the sharper signals; however, no loss of signal in the broad $\mathrm{CH}_{2} / \mathrm{CH}_{3}$ envelope was apparent even at actin excess. Previous results have indicated very little difference between the protein ${ }^{1} \mathrm{H}$-n.m.r. spectra of acto-SI and its ternary complexes with ADP or AMP.PNP. Thus, this S1.ATP analogue, which binds to actin without an apparent reduction in internal mobility, may represent a second attached state of the acto-SI complex distinct from the rigor state. (Supported by grants from MRC and SERC.)

\section{Cardiac myosins: immunological evidence for a novel isoform in nodal conduction tissue}

L. Gorza, L. E. Thornell, A. Viel and S. Schiaffino. Institute of General Pathology, University of Padova, Italy, and Department of Anatomy, University of Umeå, Sweden

Using polyclonal antibodies against bovine atrial and ventricular myosin we have previously shown that two different forms of myosin heavy chain ( $\mathrm{MHC})$, immunologically similar to the ventricular $\alpha$ and $\beta$ MHCs, are differentially distributed in the atrial myocardium [Gorza et al., J. Cell Biol. 95, 838(1982)]. We have now found that nodal muscle cells express a new type of MHC, distinct from cardiac $\alpha$ and $\beta \mathrm{MHCs}$ and immunologically related to fetal skeletal myosin. Immunofluorescence studies using anti- $\alpha \mathrm{MHC}$, anti- $\beta$ MHC and anti-bF [antibovine fetal skeletal myosin: see Sartore et al. Nature, Lond. 298, 294 (1983)] revealed the existence of different fibre populations within the nodal conduction tissue of the bovine heart. Sino-atrial and atrio-ventricular nodal fibres were all stained by anti- $a \mathrm{MHC}$, whereas only a minor proportion of fibres were labelled by anti- $\beta$ MHC antibodies; moreover, all nodal fibres displayed a specific reactivity with anti-bF. No staining with this antibody was observed in ordinary atrial and 
ventricular myocardium, except for weakly stained fibres in perinodal regions and around the orifices of the pulmonary veins. A dramatic change in reactivity was observed at the origin of the His bundle, Purkinje fibres being completely unreactive with anti-bF. In preliminary studies on fetal bovine heart we have found that even in the four-weekold embryo the regions embryologically related to nodal tissue display a specific reactivity with anti-bF, suggesting that this 'nodal myosin' may be used as a marker for studying the development of atrial conduction tissue. (Supported by grants from CNR, Gruppo Cardiorespiratorio, and Ministero Pubblica Istruzione.)

The fine structure in the diffraction spectrum obtained from rabbit psoas fibres illuminated with a laser beam

K. D. Häberle ${ }^{1,2}, R$. Rüdel ${ }^{1}, W$. Wilke ${ }^{2}$ and

F. Zite-Ferenczy ${ }^{1}$

${ }_{2}^{1}$ Abteilung Allgemeine Physiologie and

${ }^{2}$ Abteilung Experimentelle Physik der Universität Ulm, D-7900 Ulm, G.F.R.

When a frog skeletal muscle fibre is illuminated by a laser beam, the diffraction lines display a fine structure first described by Cleworth \& Edman [J. Physiol. Lond., 227, I (1972)]. The fine structure is more clearly visible in spectra obtained with glycerolated rabbit psoas muscle [Schoenberg \& Brenner, personal communication]. With a $1 \mathrm{~mm}$ beam diameter, each layer line (extending for about $15^{\circ}$ to both sides of the meridian) is composed of up to 50 streaks oriented perpendicularly to the meridian. We have interpreted this fine structure as an indication of the existence of 'columns' of myofibrils within the illuminated fibre volume, having identical sarcomere lengths (judged from the narrow meridional streak width) and with homogeneous tilt of reflecting planes with respect to the fibre axis [Rüdel \& Zite-Ferenczy, J. Physiol., Lond. 290, 317 (1979)]. The Ewald formalism allows calculation of the cross-section of such hypothetical columns from the variations of the streak length during small variations of the angle $\omega$ between beam and fibre axis. During such 'mini $\omega$-scans' streaks appeared, grew to a maximum length, then decreased and finally disappeared within $\Delta \omega=4^{\circ}-10^{\circ}$. This corresponds to 80-30 $\mu \mathrm{m}$ column diameter in the direction of the incident beam. The column diameters in the direction perpendicular to the beam, calculated from the maximum streak length, were of the same size, but in general the two dimensions were not identical for a particular streak, suggesting noncircular cross-sections. To measure the length of the columns we have followed the appearance and disappearance of streaks when the fibre was slowly moved along its axis through the beam. Streaks could usually be followed over $100-300 \mu \mathrm{m}$, i.e. somewhat less distance than that reported by Peachey \& Goldman for frog muscle [Biophys. J. 45, 102a (1984)]. The results do not support the assumption of the single myofibril acting as diffracting unit.

\section{A potentially useful probe for structural studies on actin: monoclonal antibodies directed against two isotypes of actin B. D. Hambly ${ }^{7}$, S. B. Cameron ${ }^{1}$, R. L. Raison ${ }^{2}$ and C. G. dos Remedios \\ ${ }^{1}$ Muscle Research Unit, Department of Anatomy, and ${ }^{2}$ Clinical Immunology Research Centre. The University of Sydney, Sydney. Australia 2006.}

Monoclonal antibodies directed against either skeletal or cardiac muscle actin have been generated using conventional hybridoma techniques. Balb-c mice and the NS-1 myeloma cell line were used [Hambly et al., Biochem. Int. 7, 739-46 (1983)]. Solid-phase ELISA-immunoassay experiments were performed in which the actin against which the monoclonal antibody was directed was bound to the wells of a PVC microtitre plate. After blocking with bovine serum albumin and washing, a constant quantity of monoclonal antibody was added to the wells (at a dilution corresponding to $50 \%$ of its previously determined peak titre). Additionally, increasing concentrations of competing test actin were added to the wells. After binding of the monoclonal antibody to the immobilized and/or free actin had taken place, the wells were washed and the bound antibody detected using horseradish peroxidase-conjugated sheep antimouse antibody. Two cell lines were generated against skeletal muscle actin and four against cardiac muscle actin (both alpha striated muscle isotypes). None of these antibodies was able to distinguish between these two isotypes. Further, all of these antibodies also crossreacted with squid mantle muscle actin, a beta cytoplasmic form of actin. Presumably all of the monoclonal antibodies generated in these experiments were directed against antigenic determinants that are well con- 
served through the different isotypes of actin. However it has been demonstrated previously [Morgan et al., Proc. natn. Acad. Sci. U.S.A. 77, 2069-73 (1980)] that polyclonal antisera generated against cardiac actin is capable of immunologically differentiating between skeletal and cardiac isotypes of actin. We intend to modify our technique of monoclonal antibody production, by using peptides derived from 'hypervariable' regions of actin as antigens, to obtain the greater specificity that we desire for use in probing functional sites on the actin molecule. The anti-actin monoclonal antibodies already generated will be useful as immunohistochemical probes of actin in various muscle and nonmuscle tissues.

\section{Crossbridge organization in relaxed vertebrate skeletal muscles}

J. J. Harford ${ }^{1}$, M. Cantino ${ }^{2}$ and J. M. Squire ${ }^{1}$

${ }^{1}$ Biophysics Section, Blackett Laboratory, Imperial

College, London, U.K.

${ }^{2}$ Center for Bioengineering, WD-12, Seattle,

Washington, 98195, U.S.A.

The crossbridge configuration in relaxed plaice fin muscle has been determined by computer modelling of the observed $X$-ray diffraction patterns from live whole muscles. The corresponding configuration in frog semitendinosus muscle, quick-frozen while in a relaxed state, has been determined by image analysis of electron micrographs of replicas of unidirectionally shadowed, deep-etched, freezefractured muscle fibres. The freezing was carried out using a propane jet freezing device [Cantino \& Pollack, Biophys. J., 41, 264a (1983)]. The fish muscle $X$-ray data have been found to be much easier to interpret than corresponding $X$-ray data from frog muscle. The modelling shows clear trends and the approximate crossbridge configuration is reasonably well defined. The electron micrographs from frog muscle, which have been interpreted with the aid of myosin filament models illuminated as if unidirectionally shadowed, show a different but related structure to that in fish muscle. The differences may be related to the different lattice spacings in the A-bands of the two muscles. In both cases the myosin heads lie essentially along the three helical tracks of crossbridge origins. In fish the heads are tilted at about $60^{\circ}$ to the fibre axis whereas in frog the tilt is about $30^{\circ}$. Both heads in one myosin molecule probably tilt in the same direction. Whether this is towards or away from the M-band is not yet known.
Removal and recombination of the regulatory light chains in rabbit skeletal myosin and hybridization with scallop regulatory EDTA-light chains

A. Häusermann, M. C. Schaub, D. Walzthöny and

T. Wallimann

Department of Pharmacology, University of Zürich, Switzerland, and Department of Cell Biology, Federal Technical High School of Zürich, Switzerland

In isolated myosin from rabbit fast skeletal muscles the first of the two regulatory light chains (RLC) is digested 5-10 times faster by chymotrypsin than the second one. Treatment with EDTA and DTNB removes only one of the two RLC per myosin. Interaction between the two globular head portions seems therefore to protect one of the two RLC from proteolytic attack and from removal. In actomyosin, where both myosin heads bind to actin in rigor, this steric hindrance is abolished and both RLC are equally susceptible to proteolytic attack, and both can be removed completely by treatment with chymotrypsin, EDTA and, with or without, additional DTNB. Routinely, the removal of RLC was therefore performed on actomyosin systems devoid of troponin and tropomyosin. Thereafter, myosin was dissociated from actin. DTNB in concentrations above $0.5 \mathrm{~mm}$ increases the rate of digestion of both RLC by a factor between 3 and 10, and has little effect, or rather a retardation, on some cleavage of the heavy chains. If DTNB was employed for the removal of the RLC, subsequent reductive treatment with dithiothreitol fully restored the portion of the enzymic activities which were lost due to the treatment with DTNB. Whether the RLC had been removed in the presence or absence of DTNB, the actin activated $\mathrm{Mg}$-ATPase of myosin devoid of RLC was between $45 \%$ and $65 \%$ of the normal one. The $\mathrm{Ca}$-ATPase and the K-ATPase of isolated myosin devoid of RLC were unchanged. Complete stoichiometric recombination with homologous RLC in the presence of $\mathrm{Mg}^{2+}$ and ATP fully restores the actin-activated ATPase. Also the stoichiometric recombination of the RLC, like their removal, was more readily achieved in the actomyosin system. The RLC thus seem to affect the interaction with the myosin heads with actin during the hydrolytic cycle. Electron microscope studies showed that removal of the RLC leads to a shortening of the normally ellipsoidal form of the myosin heads to a more circular shape lacking some mass in between the two heads. Further, the myosin devoid of RLC forms dimers and trimers 
preferentially by aggregation in the head region of the molecules. Reconstitution of homologous RLC leads to a decrease of the number of aggregates. These findings are corroborated by molecular sieving experiments of the same preparations on Sepharose CL-4B. It was also possible to hybridize rabbit skeletal myosin devoid of RLC with the regulatory EDTA-LC from scallop myosin. According to experimental conditions between one and two scallop EDTA-LC bound firmly to the rabbit skeletal myosin. Preliminary experiments indicate that the scallop EDTA-LC could not confer Ca-sensitivity to the actin-activated $\mathrm{Mg}$ ATPase of rabbit skeletal myosin.

\section{Localization of the nucleotide binding site of actin \\ G. Hegyi, L. Szilágyi and M. Elzinga \\ Department of Biochemistry, Eötvös L. University, \\ Budapest, Puskin u. 3, H-1088, Hungary, and \\ Department of Biology, Brookhaven National \\ Laboratory, Upton, New York, U.S.A.}

A photoactive analogue of ATP, 8-azido-ATP $\left({ }^{14} \mathrm{C}\right)$, was used as a photoactive label of the nucleotide binding site of actin. Bound ADP in F-actin was exchanged for $8-\mathrm{N}_{3}$-ATP during depolymerization. When both $8-\mathrm{N}_{3}$-ATP-G-actin and $8-\mathrm{N}_{3}-\mathrm{ADP}-\mathrm{F}$-actin (polymerized from $8-\mathrm{N}_{3}-$ ATP-G-actin) were photoactivated, covalently bound nucleotide was measured and the maximal yield was $25 \%$. After cyanogen bromide cleavage of the labelled actins, the peptides were separated as described by Elzinga [Biochemistry 10, 224-9 (1971)].

In the case of G-actin two labelled peptides were detected and purified, equal amounts of the label appeared in the peptides CB-9 and CB-16. After tryptic digestion of $\mathrm{CB}-9$ a tetrapeptide was isolated which corresponded to the Trp 356 -Ile-ThrLys $_{359}$ region of actin. The sequence analysis of the isolated peptides shows that in the tetrapeptide the label is attached to the $\operatorname{Trp}_{356}$ and in CB-16 to Lys $_{336}$.

In the case of $\mathrm{F}$-actin all the radioactive nucleotide label appeared in CB-16; the position of the label is under investigation.

Isoforms of myosin and functional state of the heart muscle in various forms of human cardiac hypertrophy

H. O. Hirzel, C. R. Tuchschmid, H. P. Krayenbuehl and M. C. Schaub

Departments of Medicine and Pharmacology,

University of Zürich, Switzerland

In hypertrophic human ventricles with various heart diseases no change was found in the peptide patterns of isolated myosin heavy chains after limited proteolysis with papain and Staphylococcus aureus V8 protease. A change in the light chain (LC) complement, however, was observed in secondary hypertrophies due to pressure overload or volume overload and in the primary dilative cardiomyopathy but not in cases of primary hypertrophic cardiomyopathy [Schaub et al., ]. Musc. Res. Cell Motility 5, 233 (1984)]. This change consists of an additional LC of the type 1 which occurs in the ventricle and which has identical electrophoretic properties to those of the atrial LC- 1 . In single cases this abnormal LC-I can be up to $30 \%$ of the total of type I LC. Still the total concentration of type I LC does not increase significantly. On average it was $0.3 \%$ in hypertrophic cardiomyopathy, $1.9 \%$ in volume overload, $5.0 \%$ in pressure overload and $12.1 \%$ in dilated cardiomyopathy. The haemodynamic parameters change characteristically in the various disease states and permit conclusions about severity and stage of the pathological conditions. The systolic wall stress reflects the load imposed to the ventricle while the mean normalized systolic ejection rate is a measure of the ventricular performance as a pump. Combination of these two parameters by taking the wall stress as a fractional portion of the ejection rate indicates that the pumping function is most depressed in primary dilative cardiomyopathy and less so in secondary forms of hypertrophy due to pressure or volume overload. No functional depression is manifest in primary hypertrophic cardiomyopathy. Plotting this latter functional parameter versus the log of the abnormal LC-I content for the mean values of the four disease groups yields a straight line with a correlation coefficient of 0.96 . Thus, the more the ventricular function becomes depressed in the presence of increasing load the higher is the content of the atrial-like LC-1 in this tissue.

\section{Immunofluorescence staining patterns of myofibrils revealed by monoclonal anti-actin antibodies}

K. Kaehn and P. Bachmann

Lehrstuhl für Anatomie I, Ruhr-Universität

Bochum, D-4630 Bochum 1, G.F.R.

We have prepared three monoclonal anti-actin antibodies from mouse hybridoma cells. Antigen specificity was tested by ELISA, immunoblotting and immunohistochemical methods. On relaxed myofibrils a fluorescence pattern of double bands is developed in I-band regions. Each double band 
unit consists of a thin unstained Z-disc and two adjacent half-fluorescent I-bands. However, with shortening of sarcomeres the immunofluorescence (IF)-staining patterns obtained with the three monoclonal anti-actin antibodies become different. Using mcab 647 and 549 the fluorescent double band in the I-band region is substituted by a single band at a sarcomere length of $2.1 \mu \mathrm{m}$; i.e. the IF-staining pattern (double/single band) of I-bands is dependent on sarcomere length. In contrast, mcab 2B5 reveals fluorescent double bands of constant width $(1 \mu \mathrm{m})$ in all stages of contraction.

At sarcomere lengths below $2 \mu \mathrm{m}$ thin filaments emerging from neighbouring Z-discs form a region of double overlap in the middle of the A-band. On myofibrils which were treated with mcab 647 a fluorescent band appears in this position. The fluorescence intensity of this newly formed band increases with further shortening of sarcomere and finally, when the thick filaments touch the $Z$-discs (sarcomere length $1.5 \mu \mathrm{m}$ ) fluorescence is exclusively located in the middle of the A-band. IF labelling of double overlap of thin filaments was not obtained using anti-actin antibodies other than mcab 647.

Conclusions: Conformational changes in the Z-disc during contraction are indicated by different IF-staining patterns of the I-band obtained either with mcab 549 or 647 . Mcab 647 and 2B5 bind to the thin filaments independent of whether they are located in the I- or A-band. We assume that the nonuniform labelling of the thin filament with mcab 647 is attributed to blocking of the epitope along the thin filament because of actomyosin crossbridges. The constant IF-staining pattern obtained with mcab 2B5 is obviously caused by an epitope which remains unaltered during contraction and which is located only on the middle section of the thin filaments. [Supported by the VW-Stiftung (1/36986).]

\section{Equilibrium constant for binding of an actin filament capping protein to the barbed end of actin filaments \\ T. Keiser and A. Wegner \\ Institut für Physiologische Chemie I. \\ Ruhr-Universität Bochum, D-4630 Bochum, G.F.R. \\ Depolymerization of treadmilling actin filaments by a capping protein isolated from bovine brain was used for determination of the equilibrium constant for binding of the capping protein to the barbed ends of actin filaments. When the capping protein blocks monomer consumption at the lengthening barbed ends, monomers continue to}

be produced at the shortening pointed ends until a new steady state is reached in which monomer production at the pointed ends is balanced by monomer consumption at the uncapped barbed ends. In this way the ratio of capped to uncapped filaments could be determined as a function of the capping protein concentration. Under the experimental conditions $(100 \mathrm{mM} \mathrm{KCl}, 2 \mathrm{mM}$ $\mathrm{MgCl}_{2}, \mathrm{pH} 7.5,37^{\circ} \mathrm{C}$ ), the binding constant was found to be about $2 \times 10^{9} \mathrm{M}^{-1}$. Capping proteins affect the actin monomer concentration only at capping protein concentrations far above the reciprocal of their binding constant. Half maximal increase of the monomer concentration requires capping of about $99 \%$ of the actin filaments. A low proportion of uncapped filaments has a great weight in determining the monomer concentration because association and dissociation reactions occur at the dynamic barbed ends with higher frequencies than at the pointed ends.

\section{Analysis of the myosin filament population in different types of muscle fibres of fish (Noemacheilus barbatulus L.)}

W. Kilarski and M. Kozłowska

Department of Comparative Anatomy,

Jagiellonian University, M. Karasia 6, 30-060

Kraków, Poland

Since the present knowledge on the composition of myosin filaments population within a given type of muscle fibre is still incomplete, we have paid particular attention to the composition of the myosin filament population in histochemically and ultrastructurally well-defined muscle fibre types. Thickness of myosin filaments was measured to an accuracy of $1.02 \mathrm{~nm}$ from electron micrographs magnified $24000 \times$. Sarcomeres of tonic, red, intermediate, transitional and white muscle fibres consist of the following three types of myosin filaments respectively: $25 \mathrm{~nm}(100 \%) ; 1.5 \mathrm{~nm}(24 \%)$, $20 \mathrm{~nm}(60 \%), 25 \mathrm{~nm}(16 \%) ; 15 \mathrm{~nm}(16 \%), 20 \mathrm{~nm}$ $(84 \%) ; 10 \mathrm{~nm}(24 \%), 15 \mathrm{~nm}(36 \%), 20 \mathrm{~nm}(40 \%)$; $10 \mathrm{~nm} \quad(72 \%), 15 \mathrm{~nm} \quad(24 \%), 20 \mathrm{~nm} \quad(4 \%)$. The observed differences in the myosin filament population may reflect the differences in the ratio of myosin isoforms contained in sarcomeres. A very homogeneous population of myosin filaments is characteristic for tonic muscle fibres. The population is composed of $25 \mathrm{~nm}$ thick filaments only. In this respect the tonic fibres resemble immature muscle fibres of fish and newts larvae [Kilarski et al. (1981)]. Sarcomeres of the red fibre muscles also contain $25 \mathrm{~nm}$ myosin filaments $(16 \%)$ in the myosin filaments population. These are the 
features typical for both undeveloped, and slow or partly slow muscle systems. Conversely the white, intermediate and transitional muscle fibres do not contain the thick $(25 \mathrm{~nm})$ myosin filaments in their sarcomeres and therefore they should be considered as forming a fully developed fast muscle system.

\section{Heavy chain-light chain and F-actin-A1 light chain interactions probed by chemical crosslinking of S1 and acto-S1 \\ J. P. Labbe, R. Bertrand, E. Audemard and \\ R. Kassab \\ Centre de Recherche de Biochimie \\ Macromoléculaire, CNRS, 34033 Montpellier \\ Cedex, France}

The influence of F-actin and nucleotide binding on the production of the 120000 heavy chain-light chain crosslinked species formed on reacting S1 $(\mathrm{A} 1+\mathrm{A} 2)$ with bis (imidoesters) was quantitatively evaluated. The crosslinking decreased to $25 \%$ of the control in the presence of actin and to $75 \%$ and $50 \%$ in the presence of $\mathrm{MgATP}$ and $\mathrm{MgADP}$ respectively. The formation of the 120000 product was accompanied for S1 (A1), but not for S1 (A2), by a loss of the actin-activated ATPase without alteration of the $\mathrm{Ca}^{2+}$-ATPase whereas the $\mathrm{Mg}^{2+}$-ATPase was increased two-fold. Up to $70 \%$, the inhibition of the acto-S1 (A1) ATPase was closely correlated with the extent of crosslinking of the A1 light chain; the activity could be largely restored upon cleavage of the crosslink. Double reciprocal plots for crosslinked and native SI (A1) showed a change in the $1 / V$ versus $1 /$ (actin) plots at actin concentrations about $4 \mu \mathrm{M}$ for the modified S1 with at least three-fold decrease of $V_{\max }$ and two-fold increase of $K_{\mathrm{app}}$. Furthermore, reaction of acto-S1 (AI) with the hydrophobic zero-length crosslinker, $N$-ethoxycarbonyl-2-ethoxy-1,2-dihydroquinoline (EEDQ) led to an extensive crosslinking between actin and A1 light chain; no crosslinking was noted between actin and the isolated light chain.

\section{Nonconventional helical muscles in \\ lumbriculids (Annelida, Oligochaeta)}

G. Lanzavecchia, M. de Eguileor, R. Valvassori and P. Lanzavecchia, Jr Dipartimento di Biologia, Università degli Studi, Via Celoria 26, 20133 Milano, Italia

Lumbriculids are medium-sized Annelids, grouped in a separate order which was generated at an early stage in the phylogenetic process, and they are apparently similar to tubificids although they are characterized by a peculiar type of motion. Longitudinal muscles are mostly ribbon-shaped (cross-section diameters: 1 and $60 \mu \mathrm{m}$ ) with interposed small triangular fibres of various aspects. The ribbon-shaped fibres are the flattened circomyarian type with very regular Z-element system and sarcoplasmic reticulum. The threedimensional interpretation of this system was obtained by computerized models, comparing the real images obtained from ad hoc sections for the three spatial planes with corresponding theoretical computerized images. On the whole, the sarcoplasmic reticulum and Z-elements form transversal pivot systems crossing the whole fibre. The fibre thus appears characterized by a rigid coordination in the contraction phases, practically acting as an old-fashioned spinning wheel. The $3 \mu \mathrm{m}$ long myosin filaments are ribbon-shaped as well, with the cross-section major axis parallel to the surface of the fibre, a thicker bare zone and tapered ends. The actin filaments are almost totally distributed in correspondence with the flattened sides of the myosin filaments forming crowns only at the tapered ends. A mechanical interpretation of the myosin filament structure is difficult to supply. It is worth stressing, however, that these filaments, in the phylum of Annelids, are found only in the species of lumbriculids so far observed; they can therefore be considered important and phylogenetically relevant taxonomic instruments.

\section{Protein recognition sites of actin: a ${ }^{1}$ H-n.m.r. spectroscopic study \\ Barry A. Levine and Arthur J. G. Moir Inorganic Chemistry Laboratory, Oxford University, Oxford OXI $3 Q R$, and Department of Biochemistry, University of Sheffield, Sheffield}

S10 2TN, U.K.

Interaction of actin with a variety of proteins is involved in the generation of motility and the control of the polymerization of actin. The conformational basis for these processes derives from the nature of the actin-actin and actin-protein contacts. The high degree of sequence homology between skeletal and cytoplasmic actins suggests strong conservation of the structural scaffold of the actin surface responsible for recognition and binding. Using ${ }^{1} \mathrm{H}$ nuclear magnetic resonance (n.m.r.) spectroscopy we have undertaken comparative studies of actin and various of its specific cleavage fragments in order to identify the surfaces of contact with actin-depolymerizing proteins such as DNAase I as well as with component proteins of the contractile assembly of skeletal muscle. 
Experiments using cyanogen bromide fragments of skeletal actin reveal complex formation between DNAase I and one of these peptides, CBIO (residues 48-82). This actin segment contains Lys-61, accessible to chemical modification only upon actin depolymerization, as well as Tyr-69 whose modification results in the inhibition of the polymerizability of actin. Analysis of the ${ }^{1} \mathrm{H}$-n.m.r. spectral effects induced by the binding DNAase I indicates the involvement of side chains of lysine (Lys-50, 61 and/or 68), tyrosine as well as hydrophobic groups of isoleucine residues in the formation of a I : I complex. Both charged and nonpolar side chains also contribute to the interaction between the peptides CB13 (residues I-44) and CB9 (residues 356-375) with skeletal myosin. The C-terminal peptide, CB9 is observed to interact with the N-terminal segment of the AI light chain on the myosin head. Experiments using intact actin indicate that troponin-I inhibits this actin-myosin linkage in a nonsteric manner which is modulated by calcium in the presence of troponin- $\mathrm{C}$ reflecting rearrangements relayed from the regions of mutual interaction. These effects can be interpreted mechanically in terms of transmittable constraints imposed on the structure of actin. (This work is supported by the MRC.)

\section{Three-dimensional structure of the fish muscle Z-line \\ P. K. Luther and J. M. Squire \\ Biophysics Section, Blackett Laboratory, Imperial \\ College, London SW7, U.K.}

The vertebrate muscle $Z$-line presents two distinct views in transverse sections, commonly referred to as 'basket-weave' and 'small-square lattice'. Much controversy has arisen over their origins and this is reflected in the various models proposed for the three-dimensional structure of the Z-line. We have studied the fish muscle $Z$-line which is very narrow axially and hence simpler than that of most vertebrate muscles [Franzini-Armstrong, J. Cell Biol. 58, 630 (1978)]. The technique of threedimensional reconstruction from tilted sections [Luther \& Crowther, Nature, 307, 566 (1984)] is being carried out in an attempt to settle this controversy. In conventional Z-line models which all possess four-fold rotational symmetry, the $(1,0)$ and $(0,1)$ views in longitudinal sections should appear identical. However, our preliminary results show that these $(1,0)$ and $(0,1)$ views of the fish Z-line are markedly different; the rotational symmetry of the Z-line is clearly two-fold and not four-fold as assumed in previous models. A new model for the fish Z-line is being developed.

Crystallization of cytoplasmic actin in complex with DNAase I and the interaction of DNAase I with polymeric actin

H. G. Mannherz ${ }^{1}$, J. Dieckhoff ${ }^{1}$, D. Suck ${ }_{4}{ }^{2}$

W. Kabsch ${ }^{3}, K$. Friebel ${ }^{4}$ and M. Frimmer ${ }^{4}$

${ }^{I}$ Institute for Anatomy and Cell Biology, Marburg,

${ }^{2} E M B L$, Heidelberg, ${ }^{3}$ MPI for Medical Research,

Heidelberg, and ${ }^{4}$ Institute for Pharmacology and

Toxicology, Gießen, F.R.G.

Crystals of porcine liver actin in complex with DNAase I have been obtained. These crystals are of identical habit and space group to the crystals obtained from rabbit skeletal muscle actin in complex with DNAase I. By difference Fourier analysis no structural difference could be detected up to $0.6 \mathrm{~nm}$ resolution of this cytoplasmic actin to its skeletal muscle counterpart. Heavy atom substitution of the crystalline cytoplasmic actin-DNAase I complex with methyl-mercuriacetate (MMA) was found not to be possible. Since in both skeletal and cytoplasmic crystalline actin-DNAase I complexes the C-terminal cystein (No. 374) had been removed prior to crystallization by mild tryptic treatment, it is concluded that the sulphydryl labelled by MMA in skeletal muscle actin is located at position No. 10. This assumption is supported by results indicating that the reactivity of cytoplasmic and smooth muscle but not of skeletal muscle actin versus DTNB is reduced after complex formation with DNAase I. At the recently obtained map of $0.45 \mathrm{~nm}$ resolution of crystalline skeletal muscle actin-DNAase I complex the MMA was found to be localized within the Nterminal peptide which is found within the smaller domain of the two discerned at $0.6 \mathrm{~nm}$ resolution.

Chemically crosslinked actin was used to study the interaction of polymeric actin with DNAase I. It was found that polymeric actin is also able to inhibit the DNA-degrading activity of DNAase I after prolonged incubation to an almost identical degree as G-actin. These results are taken as preliminary evidence that DNAase I can also interact with actin monomers which are chemically stabilized in the polymeric form. It was furthermore found that bovine pancreatic DNAase I is able to block the fast growing end of fractionated chemically crosslinked actin oligomers and trimers, thus inhibiting their capacity to nucleate actin polymerization and Cytochalasin B binding. This property is also exhibited by rat parotid DNAase I, 
which is otherwise unable to depolymerize F-actin and binds to monomeric actin with an affinity of about two orders of magnitude lower than bovine pancreatic DNAase I.

\section{Localization of dansyl-reactive lysyl residues along the heavy chain of myosin subfragment-1}

L. Nyitray and M. Bálint

Department of Biochemistry, Eötoös L. University, Budapest, Puskin u. 3, H-1088, Hungary

1-Dimethylaminonaphthalene-5-sulphonyl chloride (Dns-) was used for chemical modification of myosin subfragment-1 (SI). The incorporation of Dns group was $2 \mathrm{~mol} \mathrm{~mol}^{-1} \mathrm{~S} 1$ accompanied by loss of the $\mathrm{K}^{+}-\mathrm{EDTA}$-ATPase activity and the decrease in $\mathrm{Ca}^{2+}$-ATPase activity. However, the modification of SI by Dns-Cl does not resemble an active site directed process because neither MgATP nor MgADP protect against inactivation.

Dns groups were predominantly attached to lysyl residues as identified on two-dimensional chromatogram of $\mathrm{HCl}$ hydrolysate of dansylated S1. It was found that the two dansyl-reactive lysyl residues were located in the heavy chain of the native S1. Limited tryptic fragmentation of dansylated SI produced the same positioned peptides as that of native S1: $25 \mathrm{~K}-50 \mathrm{~K}-20 \mathrm{~K}$. One of the dansyl-reactive lysines (DRL-I) was located in the $\mathrm{N}$-terminal $25 \mathrm{~K}$ fragment, while the other one (DRL-2) was in the loose C-terminal end of chymotryptic SI heavy chain which could be eliminated by trypsin. The peptide containing DRL-2 may possibly come from the flexible, protease-sensitive swivel connecting SI to myosin rod. We showed that DRL-2 was located at the $20 \mathrm{~K}-37 \mathrm{~K}$ junction in trypsin-digested heavy meromyosin, i.e. it was found in the $60 \mathrm{~K}$ precursor but was absent in the descendant $20 \mathrm{~K}$ and $37 \mathrm{~K}$ (subunit of short subfragment-2) fragments.

It is suggested that DRL-I is different from the well-known TNBS-reactive lysyl residue which is also located in the $25 \mathrm{~K}$ fragment [Mornet et al., FEBS Lett. 117, 183 (1980)], because: (1) trinitrophenylation was accompanied by marked increase in the $\mathrm{Mg}^{2+}$-ATPase activity in contrast to dansylation; (2) the same incorporation and localization of Dns groups were obtained in the case of TNP-SI. DRL-2 may be related to one of the reactive lysyl residues localized in the swivel region of cardiac myosin and which are preferentially labelled with an anilinonaphthalenesulphonyl derivative [Hiratsuka \& Uchida, ]. Biochem. 89, 111 (1981)].

A comparison of myosin isoenzymes in hindlimb and forelimb muscles of normal and genetically dystrophic $\left(\mathrm{C} 57 \mathrm{BL} / 6 \mathrm{~J} \mathrm{dy} / \mathrm{dy}{ }^{2 J}\right)$ mice

D. J. Parry and A. Stewart

Department of Physiology, University of Ottawa,

Ontario, Canada K1H 8M5

We have previously shown, with an immunohistochemical technique, that the fast-twitch anterior tibialis (AT) and extensor digitorum longus (EDL) muscles of normal adult mice consist almost exclusively of fibres which contain fast myosin (no reaction with anti-cat soleus myosin). By contrast, in these muscles of dystrophic mice many fibres were seen to contain slow myosin [Parry \& Desypris, Musc. Nerve 6, 397-407 (1983)]. A similar shift in isoenzyme composition in hindlimb muscles of another strain of dystrophic mice (Re J 129 dy/dy) was recently reported by Fitzsimons \& Hoh [J. Physiol, Lond. 343, 539-50(1983)], using nondenaturing pyrophosphate gels. We have suggested that the synthesis of slow myosin in these normally fast-twitch muscles is a result of the spontaneous activity to which the hindlimbs are subject rather than the dystrophic process itself. The reasons for this suggestion are, (I) slow myosin appears several weeks after the appearance of histological signs of dystrophy and seems to follow the onset of fused spontaneous contractions, (2) slow myosin is not seen in the forelimb muscles which are not spontaneously active. Since immunohistochemical techniques reveal the nature of myosin heavy chains we have used SDS-PAGE to determine the distribution of myosin light chains in hindlimb and forelimb muscles of normal and dystrophic mice. Myosin was extracted from muscles pooled from several mice of the same age by a modification of the method of Dalla Libera et al. [Musc. Nerve 1 , 280-91 (1978)]. As expected, both AT and the fast-twitch forelimb muscle, extensor carpi radialis (ECR) of control mice contained the three light chains $L C_{1 f}, L_{2 f}$ and $L_{3 f}$. However, AT of dystrophic mice was found to contain $L S_{1 S}$ and $\mathrm{LC}_{2 S}$ in addition to the three 'fast' light chains. In these preliminary experiments it seemed that $\mathrm{LC}_{1 \mathrm{~S}}$ appeared somewhat earlier than $\mathrm{LC}_{2 \mathrm{~S}}$. In ECR of dystrophic mice neither $\mathrm{LC}_{1 S}$ nor $\mathrm{LC}_{2 S}$ was seen. Thus, it appears that the shift in myosin heavy chain isoform is accompanied by at least a partial shift in the distribution of myosin light chains due to the altered pattern of activity. 
Actin: antigenic and functional domains

C. Roustan, $Y$. Benyamin and M. Boyer

Centre de Recherches de Biochimie

Macromoléculaire, CNRS, BP 5051 F-34033

Montpellier Cedex, France

The involvement of actin microfilaments in cellular motility is now well documented. Numerous proteins, are associated with and modulate the state of actin. These changes affect filament numbers, length and interaction with each other or cellular structure. Furthermore it has been shown, in vitro, that numerous steps are necessary in the self association of actin monomers leading to filament formation.

We have focused our investigations on the transition step induced by $\mathrm{Mg}^{2+}$. An immunological approach was chosen. Two lots of anti-actin were selected. The first one, which is preferentially directed towards the $\mathrm{N}$-terminal sequence of actin, does not affect actin polymerization. In contrast, the second one, which is essentially directed towards the central part of actin sequence prevents actin polymerization. In connection, $15 \%$ of these antibodies are released during the transition which occurs when $\mathrm{G}$-actin binds $\mathrm{Mg}^{2+}$. The specificity of the released antibodies is restricted to the $168-226$ region in the primary structure of G-actin.

In conclusion, we report that a located conformation change occurs in actin monomer upon $\mathrm{Mg}^{2+}$ binding, conformation change which would constitute one essential step in the $\mathrm{Mg}^{2+}$ actin polymerization process.

\section{Myosin isoforms in the skeletal muscle of lower vertebrates}

A. Rowlerson ${ }^{1}$ and A. Veggetti ${ }^{2}$

'Department of Physiology, Glasgow University, U.K.

2Istituto di Anatomia degli Animali Domestici, Università di Bologna, Italy

In contrast to mammalian and avian skeletal muscle myosins, the myosins of lower vertebrates have been little investigated. We and other colleagues are now making a comparative study of myosins in the skeletal muscles of fish, amphibia and reptiles, using a combination of histo- and immunohistochemical and electrophoretic techniques. The division of amphibian muscle fibres into tonic and twitch types has long been recognized, but our recent results show that, using the histo- and immunohistochemical methods, it is possible to identify four myosin types in the frog and toad. The frog has only one type of 'slow' myosin (found in the tonic fibres) and three types of 'fast' myosin, whereas the toad has two types of 'slow' myosin, one as in the frog and another which is both histo- and immunohistochemically distinct. There are also at least two types of 'fast' myosin in the toad. Two kinds of 'slow' myosin exist also in the snake, and preliminary evidence suggests that a similar situation may occur in the lizard and tortoise. Distinctions between fast myosins in the reptiles is harder to obtain as, with one exception, they react poorly with all the anti-fast myosin sera we use, which were raised against fish, avian and mammalian myosins.

Myosins from the slow (red) and fast (white) muscle of fish differ in both their heavy chains (HC) and light chains (LC). Whereas fish slow LC resemble mammalian slow LC in both molecular weight and pI, fish LCIf is characteristically much more acid in pl than the mammalian LCIf, and the variable migration of fish LC3F in the second dimension of 2-D gels is species dependent. An analysis of the myosin LC composition of single fibres of fish muscle has also shown that, as in the case of some mammalian fibres, some slow fibres contain a small proportion of LCIf and LC3f in addition to the slow LC.

Antisera specific for fish fast myosin fail to distinguish between pink and white muscle myosins, which have the same fast LC; however, there is evidence for a distinct pink muscle myosin $\mathrm{HC}$ type in some fish. [Work supported by the S.E.R.C. (grant to Dr N. C. Spurway) and the M.P.J. (40\%).]

\section{Pyridoxal $5^{\prime}$-phosphate modifies lysines in myosin subfragment- 1 responsible for actin binding}

É. Sárközi and L. Szilágyi

Department of Biochemistry, Eötvös L. University,

Budapest, Puskin u. 3. H-1088, Hungary

Pyridoxal 5'-phosphate (PLP) is a specific and effective reagent for the essential lysyl residues in various dehydrogenases, kinases and other enzymes. Under relatively unfavourable conditions ( $\mathrm{pH}$ 7.0) about two lysines can be modified in the myosin subfragment-1 by PLP. The specificity of PLP is strikingly different from that of TNBS since the reactive lysine residue (RLR) remains unmodified in this reaction. Three lines of evidence led us to these conclusions. (I) The ATPase activities $\left(\mathrm{K}-\mathrm{EDTA}, \mathrm{Ca}^{2+}\right.$ and $\mathrm{Mg}^{2+}$ ) of pyridoxylated S1 do not change. (2) The kinetics of the incorporation of trinitrophenyl group followed spectrophotometrically are identical in the case of unmodified and PLP-treated S1. The changes in the ATPase 
activities of the double modified SI are characteristic of the modification of RLR in S1. (3) Trinitrophenylation of the RLR in SI decreases the rate of the nucleotide-induced tryptic fragmentation of the N-terminal 27000 fragment into a 22000 one. No such effect was observed after PLP treatment.

The actin-activated $\mathrm{Mg}^{2+}$-ATPase activity of subfragment-1 decreases on account of PLP incorporation. Double reciprocal plot of activity versus actin concentration shows no change in the $V_{\max }$. On the other hand the association constant $\left(K_{\mathrm{app}}\right)$ increased from $36 \mu \mathrm{M}$ (unmodified S1) to $90 \mu \mathrm{M}$ as a result of the modification of two lysines by PLP. This implies that the modification occurs at a part of the subfragment- 1 molecule which is involved in the binding of actin.

\section{Anomalous ventricular light chain-1 in human cardiomyopathy}

F. A. Sreter, K. Mabuchi, L. Papp, P. Sotonyi,

P. Allen and J. Gergely

Boston Biomedical Research Institute,

Massachusetts General Hospital, Brigham and

Women's Hospital, Harvard Medical School,

Boston, Massachusetts, U.S.A., and Semmelweis

Medical School, Budapest, Hungary

Previous work has indicated the presence of ventricular type myosin light chains in atria [Cummins, Biochem. J. 205, 195 (1982)] and atrial type light chains in ventricles of hypertrophied human heart [Tuchschmid et al., in Cardiac Adaptation to Hemo-dynamic Overload, Training and Stress, Steinkopff, p. 123 (1982); Streter et al,, ibid., p. 129]. Another group of investigators found no change in the light chain complement of hearts of patients with hypertrophic cardiomyopathy [Schier \& Adelstein, J. clin. Invest. 69, 816, (1982)]. In this work myosin extracted from human left ventricular biopsy samples of cardiomyopathic (CMP) hearts, dilated or hypertrophic but without ischaemic disease, or from removed hearts of recipients of heart transplants, was studied. Myosin was run on nondissociating pyrophosphate-containing gels and the isoenzyme bands were cut out and rechromatographed on SDS slab gels. The light chain pattern indicated the presence of a light chain whose mobility was faster than that of the ventricular VLC1. The mobility of VLC2 of CMP hearts was in some samples somewhat slower than that of normal VLC2. It appears that the $\mathrm{N}$-terminus of CMP VLCI is blocked, suggesting that if the anomalous light chain is related to that found in normal ventricles the N-termini are presumably identical. It also suggests that, if the anomalous light chain was a breakdown product of normal LC1 the cleavage could not have occurred at the N-terminus. The gels showed hardly any indication of proteolytic breakdown, making this possibility unlikely. Polyclonal antibody raised against normal VLCI reacted also with VLCI found in CMP hearts. Digests with trypsin of trypsin + papain of the isolated CMP VLCI produced a pattern different from that of normal VLC1. Immunoblasts of the CMP digests showed distinct differences from the normal ones. A reasonable preliminary interpretation of our data is that in cardiomyopathic ventricle a gene not expressed in normal ventricle is active. At present we are following up indications that the anomalous light chain may be identical with the LCI of slow skeletal muscle. (Supported by grants from NTH, NSF and MDA.)

\section{The rapid and high effective method of purification of cardiac troponin $\mathrm{C}$}

J. Szynkiewicz, D. Stepkowski, H. Brzeska and

W. Drabikowski

Nencki Institute of Experimental Biology, Warsaw, Poland

The aim of the present study was to develop a rapid and effective procedure for the preparation of cardiac troponin C (CTNC) of a high purity and low content of calmodulin. The methods of purification available so far give a low yield ( $15 \mathrm{mg}$ $\mathrm{kg}^{-1}$ of muscle) of cTNC usually contaminated by calmodulin. In our purification procedure three main steps are involved. In the first step calmodulin and the other proteins are removed from muscle homogenate by EDTA treatment. Then in the second step the residue is suspended in $6 \mathrm{M}$ urea and CTNC present in a soluble form is bound to a basic ion exchanger in the presence of $0.2 \mathrm{M} \mathrm{KCl}$. Proteins less acidic than CTNC are not bound to Sephadex under these conditions. The cTNC is removed from the Sephadex in the solution containing $6 \mathrm{M}$ urea and $0.8 \mathrm{M} \mathrm{KCl}$. The third step is based on the observations that calcium-binding proteins can be hydrophobically bound to phenylSepharose column in the presence of calcium and eluted by EDTA-containing solution, which allows the cTNC from residue proteins and nucleotide containing material to be purified.

Cardiac TNC obtained according to our method is of a high purity as shown by polyacrylamide gel electrophoresis, u.v. absorption and fluorescence spectra. Phosphodiesterase test shows less than $0.1 \%$ of contamination of cTNC by calmodulin. 
The ability of cTNC to form a complex with TNI and the conformational changes caused by binding of calcium ions were estimated by u.v. absorption, circular dichroism, fluorescence spectra and mobility of protein in the polyacrylamide gel. The method described above has several advantages compared to methods published earlier. It allows a high yield of cTNC (160 $\mathrm{mg} \mathrm{kg}^{-1}$ of muscle) to be obtained during a short purification procedure; also contamination by calmodulin, and nucleotide containing material is negligible.

New aspects on the structure and composition of the myofibrillar M-band

L.-E. Thornell, E. Carlsson, E. Kugelberg,

B. K. Grove and H. M. Eppenberger

Department of Anatomy, University of Umeà,

S-901 87 Umeå, Sweden, Department of

Neurology, Karolinska Hospital, S-104 01

Stockholm, Sweden, and Institute for Cell Biology,

ETH, CH-8093 Zürich, Switzerland

The myofibrillar M-band is in current structural models thought to be composed of M-bridges, $M$ filaments and secondary $\mathrm{M}$-bridges in addition to being the middle part of the bare region of the thick filaments. Three proteins have been identified as constituents of the $\mathrm{M}$-band: $\mathrm{M}$-creatine kinase (MCK) $\left(M_{\mathrm{r}} 40000\right), \mathrm{M}$-protein $\left(M_{\mathrm{r}} \mathrm{I} 65000\right)$ and myomesin $\left(M_{\mathrm{r}} 185000\right)$. MCK is thought to form M-bridges while the exact localization of the other two proteins is unknown. By the use of polyclonal antibodies against MCK, and monoclonal antibodies against $M$-protein and against myomesin, in combination with light and electron microscopic immunocytochemical techniques including cryoultramicrotomy, we have studied the localization of the M-band proteins in various skeletal muscles and species. Furthermore, fibres of electrophysiologically, histochemically and ultrastructurally characterized motor units from the rat soleus muscle have been investigated.

Our studies show that all muscle fibres so far examined contained myomesin irrespective of fibre type and species. Thus myomesin seems to be a ubiquitous protein of the myofibrillar M-band. The presence of $\mathrm{M}$-protein on the other hand was related to fibre types, e.g. type IIA fibres were the only labelled fibres in chicken ALD while both type IIA and type IIB fibres were positively labelled in the white portion of the chicken pectoralis muscle. In the rat soleus muscle all fast twitch fibres (contraction times of $16-19 \mathrm{~ms}$, histochemically type IIA fibres) were labelled with antibodies against $\mathrm{M}$-protein while fibres of slow twitch motor units (contraction times of $30-37 \mathrm{~ms}$, histochemically type I fibres) were nonreactive. Fibres of motor units under transformation (contraction times $23-27 \mathrm{~ms}$, histochemically type IIC fibres), showed weak to moderate reaction with the same antibodies. Furthermore, as all the fibres in the rat soleus at birth showed equally strong positive reaction with antibodies against $M$-protein, the nonreactivity in slow twitch fibres seems to be related to differentiation and specialization. Ultrastructurally, the fast twitch fibres showed a five-line pattern in the M-band while the slow twitch fibres had a four-line pattern.

These examples indicate a great complexity of the M-band. However, with the present tools new insight into the correlation between M-band composition and structure, and fibre type characteristics may be obtained.

\section{Differential binding of rabbit fast muscle subfragment- 1 isoenzymes to regulated actin in the presence of $\mathrm{Ca}^{2+}$}

Hylary R. Trayer ${ }^{1}$, Barry A. Levine ${ }^{2}$ and

Ian P. Trayer ${ }^{1}$

${ }^{t}$ Department of Biochemistry, University of

Birmingham, Birmingham B15 2TT, U.K.

${ }^{2}$ Inorganic Chemistry Laboratory, University of

Oxford, Oxford OX1 $3 Q R, U . K$.

Reports from various laboratories have shown that the alkali light chain isoenzymes of rabbit fast muscle myosin SI [SI(AI) and SI(A2)] differ: (I) in their affinities for immobilized actin and (2) in the actin-activated ATPase activities. These differences are only expressed at low ionic strength (ca. $\mu<0.02 \mathrm{M})$ and disappear when this is raised. Nevertheless, several lines of study indicate that the Ala, Pro, Lys-rich cluster of amino acids at the $\mathrm{N}$-terminus of the AI light chain interact directly with actin in the acto-SI(A1) complex even at physiological ionic strength. In this report the direct binding of $\mathrm{SI}(\mathrm{AI})$ and $\mathrm{SI}\left(\mathrm{A}_{2}\right)$ to actin and regulated actin (i.e. actin + tropomyosin + troponin) has been investigated by centrifugation studies in the Airfuge. The same qualitative results were found when the concentration of SI was determined by measurement of the $\mathrm{NH}_{4}^{+}$-ATPase activity (using unlabelled proteins) or by fluorescence (using $\mathrm{SH}_{\mathrm{I}}$-fluorophore labelled SI). Binding was measured in the presence of either Mg.AMP.PNP $\left(\mu=0.07 \mathrm{M}, 24^{\circ} \mathrm{C}\right)$ or Mg.ADP $\left(\mu=0.27 \mathrm{M}, 24^{\circ} \mathrm{C}\right)$. It was found that under these conditions there was no difference between the binding affinities of $S 1(A 1)$ and $S I(A 2)$ for F-actin. In contrast, SI(A1).ADP and S1(A1).AMP.PNP 
were found to bind consistently more tightly to regulated actin in the presence of $\mathrm{Ca}^{2}+$ than SI(A2).nucleotide. This difference in the binding affinities of the two isoenzymes for regulated actin was considerably diminished in the absence of $\mathrm{Ca}^{2+}$ although not entirely eliminated.

In separate ${ }^{1} \mathrm{H}$-n.m.r. studies it was found that the binding of troponin I to actin specifically weakens the interaction between the $\mathrm{N}$-terminal segment of the AI light chain in SI(AI) and actin without affecting the heavy chain interaction site(s). These data suggest a modifying role for the N-terminal region of the AI light chain in regulation of the contractile process. (Supported by grants from The Wellcome Trust and MRC.)

\section{Myosin head configurations and stem mass determinations \\ Doris Walzthöny ${ }^{1}$, Martin Bähler ${ }^{1}$, \\ Andreas Engel ${ }^{2}$, Theo Wallimann ${ }^{1}$ and \\ Hans M. Eppenberger ${ }^{1}$ \\ ${ }^{1}$ Institut für Zellbiologie, ETH, Hönggerberg, \\ CH-8093 Zürich, Switzerland \\ ${ }^{2}$ Biozentrum der Universität Basel, Klingelbergstr. \\ 70, CH-4056 Basel, Switzerland}

Myosin molecules were directly visualized without heavy metal shadowing by scanning transmission electron microscopy (STEM) under low dose conditions. The general appearance and dimensions of heavy metal-free molecules were similar to those of shadowed myosin, either after freeze-drying without or air-drying with glycerol. Two characteristic configurations of myosin head regions were found, a first type showing two pear-shaped heads with narrow necks and a second type showing a distinctly trinodular structure with two heads and extra mass in the neck region. The mass of the trinodular type as determined by STEM $\left(M_{r}=265 \pm 39\right)$ is in excellent accordance with biochemical data $\left(M_{\mathrm{r}}=258,263,268\right.$, depending on the alkali light chain isoforms), whereas the mass of the pear-shaped type is somewhat lower $\left\langle M_{\mathrm{r}}=210 \pm 44\right)$ [Walzthöny et al., EMBO J. 3 (in press)].

The extra mass in the neck region of the trinodular type seems to represent the regulatory domain [Winkelmann et al., Nature, Lond. 307, 758 (1984)\} where head to head communication is achieved via inter-head light chain interactions and where conformational changes take place upon activation [Hardwicke et al, Nature, Lond. 301, 478 (1983)].

The trinodular head configuration probably represents a more realistic structure of myosin than the pear-shaped heads. The latter lack the central domain and seem to have lost some mass [presumably DTNB light chains(s)] during the preparation for EM, presumably during dehydration. This is supported by the fact that the regulatory or DTNB light chains which are located in the neck region and extend into the SI-S2 junction [Flicker et al., J. molec. Biol. 169, 723 (1983); Winkelmann et al., Cell 34, 295 (1983); Winkelmann et al., Nature, Lond. 307, 758 (1983)] are relatively easily removed.

\section{Similar affinities of ADP and ATP for G-actin at physiological salt concentrations}

M. Wanger

Institut für Physiologische Chemi I,

Ruhruniversität, Universitätsstrasse 150, D-4630

Bochum, F.R.G.

G-actin bound ATP prevents denaturation of Gactin and is hydrolysed by polymerized actin to form ADP. This reaction is a prerequisite for the treadmilling process of actin filaments. Given the importance of the nucleotide for the function of actin the exchange of ADP and ATP at G-actin was investigated previously. These studies have been performed at low salt concentrations mostly with $\mathrm{Ca}^{2+}$ as the divalent cation. The values for the relative equilibrium constants for the ADP/ATP exchange range from 100 [Seidel $e t$ al., Biochim. Biophys. Acta 140, 93-108 (1967)] to 175 [Neidl \& Engel, Eur. J. Biochem. 101, I63-9 (1979)] in $0.8 \mathrm{mmol} \mathrm{l}^{-1} \mathrm{Ca}^{2+}$.

I have investigated the exchange of the fluorescent ATP-analogue 1- $\mathrm{N}^{6}$-ethenoadenosine- $5^{t}$ triphosphate [E-ATP, Secrist et al., Biochemistry 11, 3499-506 (1972)] and ATP or ADP at G-actin by fluorescence titration. The ADP/ATP exchange constant was calculated from these data. The studies "show that the exchange constant strongly depends on the salt conditions. At $37^{\circ} \mathrm{C}, \mathrm{pH} 7.5$ and $0.8 \mathrm{mmol}^{-1} \mathrm{Ca}^{2+}$ it was found to be 110 , in agreement with the previously reported results; however, it was only 3 at physiologically relevant salt concentrations $\left(37^{\circ} \mathrm{C}\right.$ pH $7.5,0.8 \mathrm{mmol} \mathrm{l}^{-1}$

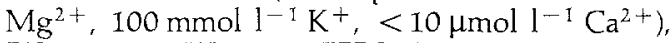
[Wanger \& Wegner, FEBS Lett. 162, 112-16 (1983)].

\section{Molecular packing in myosin rod paracrystals}

R. Ward and P. M. Bennett

M.R.C. Cell Biophysics Unit, 26-29 Drury Lane, London WC2, U.K.

To investigate the packing of myosin tails in the thick filament shaft, the aggregation of isolated 
tails, myosin rod, has been studied using electron microscopy and optical diffraction. We have previously shown that myosin rod from chymotryptic digestions of vertebrate skeletal myosin has the same length as the myosin tail. When aggregated by reduction of the ionic environment to $0.1 \mathrm{M}$ salt at $\mathrm{pH} 7.0$, paracrystalline flat ribbons, up to $30 \mu \mathrm{m}$ long, are produced. Metal shadowing reveals a fine $14.3 \mathrm{~nm}$ axial repeat on the surface. The paracrystals are sensitive to conditions of very low ionic strength, and split up into many protofilaments of the same size as the subfilaments found by Maw \& Rowe [Nature, Lond. 286, 412 (1981)] in thick filaments. Also sections of the paracrystals show a structure with subunits of the same size.

Optical diffraction of micrographs of negatively stained paracrystals show a strong $1 / 43.0 \mathrm{~nm}^{-1}$ meridional reflection, and higher orders. In patterns from some micrographs there are also a set of layer lines indexing at $1 / 43.0 \mathrm{~nm}^{-1}$ with sampling on row lines at orders of $1 / 12.5 \mathrm{~nm}^{-1}$. Some patterns extend to $1 / 3 \mathrm{~nm}^{-1}$ on the equator. Computer filtered images of some areas of ribbons have been produced that reveal a structure with weaving subfilaments at least $6 \mathrm{~nm}$ in diameter, perhaps equivalent to those reported by Maw \& Rowe.

\section{FIBRE-TYPE MODULATION AND REGENERATION}

Fibre histochemistry and contractile properties of mouse mutant skeletal muscles

E.-M. Füchtbauer, J. Reininghaus and H. Jockusch

Developmental Biology Unit, W7, University of

Bielefeld, POB 8640, D-4800 Bielefeld I, F.R.G.

A shift in fibre type proportions had been observed in several hereditary neuromuscular diseases in humans [cf: Fardeau et al., In Current Topics in Nerve and Muscle Research. (edited by Aguayo and Karpati), pp. 164-77. Amsterdam: Elsevier (1978)]. With this in mind we have investigated three mutations in the mouse that affect the maturation and functional maintenance of motor units : 'wobbler' (wr), 'jolting' (med $\left.{ }^{\mathrm{j} o}\right)$, a mild allele of 'motor endplate disease' (med), [Sidman et al., Ann. N.Y. Acad. Sci. 317, 479-505 (1979)] and 'arrested development of righting response' (adr) [Watts et al., In The Biochemistry of Myasthenia Gravis and Muscular Dystrophy (edited by Marchbanks Lunt), pp. 309-13, London: Academic Press (1978)]. All of these show severely abnormal motor behaviour and reduced muscle masses. Isometric contractions of the fast twitch muscle, tibialis anterior, were measured in situ using direct stimulation. Single twitches of all three mutants were normal up to an age of 60 days, only those of older ( $\geqslant 80$ days) adr and wr individuals were prolonged, by factors of 1.3 and 2.5 , respectively. Tetanic contractions were normal for wr and medio, but long-lasting after-contractions were observed with adr muscles from 17 days onwards. Enzyme histochemistry (myosin ATPase, EC 3.6.1.3, after preincubation at $\mathrm{pH} 4.60$, succinic dehydrogenase SDH, EC 1.3.99.1 and $a$-glycerophosphate dehydrogenase GPDH, EC 1.1.99.5) was performed on frozen sections of tibialis anterior plus extensor digitorum longus and gastrocnemius plus soleus. In all three mutants there was no obvious abnormality in ATPase fibre type patterns, indicating that the proportions of type I (slow) and type II A and B (fast) fibres were not changed, in accordance with the single twitch measurements. While SDH and GPDH stainings revealed 'checkerboard' patterns in wr and medio, the staining was homogeneous and very much like that of the soleus in adr fast muscle (Watts et al, 1978). This abnormality in oxidative/glycolytic enzyme levels in the adr mutant is paralleled by a drastic reduction in the $\mathrm{Ca}^{2+}$ binding protein, parvalbumin [Stuhlfauth et al., Proc. natn. Acad. Sci. U.S.A. 81, 4814-18 (1984)]. At present it is not known how these biochemical changes in adr muscle are connected to its unusual contraction behaviour. (This work is supported by Deutsche Forschungsgemeinschaft, grant DFG Jo 84/7.)

\section{Ectopic transplants of mammalian atrial and ventricular tissue: physiology and histochemistry}

H. Jockusch, E.-M. Füchtbauer, A. Füchtbauer, G. Mehrke, H. Deschner and K. Bertram Developmental Biology Unit, W7, University of Bielefeld, POB 8640, D-4800 Bielefeld 1. F.R.G. We have reported on the regeneration of newborn rat ventricular tissue transplanted ectopically into a skeletal muscle bed of the nude mouse [Jockusch et 
al., Expl Neurol. 81, 749-55 (1983)]. In the present communication we extend our observations on atrial tissue and on a subcutaneous transplantation site in order to study (1) the stability of the differentiated state of cardiac cells in an ectopic environment, (2) possible competition, mutual enhancement or other interactions with neighbouring muscle or connective tissue and (3) the possibility of functional innervation by a motor nerve.

Our results may be summarized as follows. (I) Cardiac muscle organization, the formation of blood filled spaces and autonomous beating activity are displayed both by atrial and ventricular tissue, in the muscle bed as well as the subcutaneous site. Even single displaced cardiac cells surrounded by skeletal muscle fibres remain 'cardiac' as demonstrated by an antibody against heart specific $a$-myosin heavy chain [anti-V1, kindly provided by Dr K. Schwartz, Paris; cf. Schwartz et al., J. biol. Chem. 257, 14412-I8 (1982)]. (2) Atrial muscle has a higher potential to regenerate and is more active in autonomous pulsation than ventricular muscle. Furthermore, 38 days after transplantation to an ectopic site (muscle bed or subcutaneous), atrial muscle cells can be distinguished from ventricular cells by their specific secretory granules [Jamieson \& Palade, J. Cell Biol. 23, 151-72 (1964)]. Thus regional ultrastructural differences are retained in ectopic heart muscle. (3) At present, there is no evidence of functional innervation by cholinergic motor nerves in the skeletal muscle bed. (This work is supported by Deutsche Forschungsgemeinschaft, grant DFG Jo 84/7.)

\section{Parvalbumin levels and in vitro translation of its mRNA in chronically stimulated rabbit muscle \\ E. Leberer, U. Seedorf, G. Klug and D. Pette \\ Fakultät für Biologie, Universität Konstanz, \\ Postfach 5560, D-7750 Konstanz, F.R.G.}

As was previously shown, fast to slow transformation of rabbit muscle by chronic stimulation leads to a rapid decrease in parvalbumin (PA) concentration [Klug et al., Pflügers Arch. 399, 280-4 (1983)]. The aim of the present study was to correlate the time course of PA decay with that of the decreases of its mRNA. PA was quantified by an ELISA in homogenates of extensor digitorum longus muscles from rabbits which were stimulated ( $10 \mathrm{~Hz}, 12 \mathrm{~h} /$ day) from 2 to 52 days. mRNA was purified from the same homogenates and was translated in vitro by using the reticulocyte lysate system. Translated PA was immunoprecipitated and quantified after electrophoresis by measuring ${ }^{35}$ S]methionine incorporation. The following results were obtained. Translatable mRNA coding for PA decreases significantly in muscles stimulated more than two days. A rapid decrease to $30 \%$ of its original level occurs between two and eight days of stimulation. The decay levels off thereafter. After 21 days stimulation, mRNA coding for PA has reached $10 \%$ of its concentration in unstimulated fast-twitch muscle. Prolonged stimulation does not lead to a further decrease. Similarly to its mRNA, PA is found to decrease rapidly in the stimulated muscle. However, its decay follows that of the mRNA with a delay of two days. Thus, PA begins to decrease significantly after six days. Its decrease is steady until 21 days when it reaches about $5 \%$ of its concentration in normal fast-twitch muscle. Taken together, these results indicate that the rapid decrease of PA during the fast to slow transformation of chronically stimulated muscle is primarily due to a reduced concentration of its mRNA. It appears that this decrease is due to a reduced transcription of the PA gene. The fact that prolonged stimulation does not reduce mRNA below $10 \%$ of its original value, whereas the PA concentration in the stimulated muscle decreases to $5 \%$, may be related to the fact that proteins in slow-twitch muscles are generally characterized by higher turnover rates. (Supported by Deutsche Forschungsgemeinschaft, Sonderforschungsbereich 138 und Sonderforschungsbereich 156.)

\section{Isozymes of myosin in regenerating rat muscles}

G. Maréchal, Ketty Schwartz, Godelieve

Beckers-Bleukx and E. Ghins

Department of Physiology, University of Louvain, B-1200 Brussels, Belgium, and U 127, INSERM,

\section{Paris, France}

Native myosin isozymes of rat muscle have been isolated by electrophoreses in nondissociating conditions. Their mobilities were measured, using taenia coli as an internal standard and their relative concentrations were determined by computer planimetry of the electrophoretograms. Three isozymes were observed in adult extensor digitorum longus, two in adult soleus, four in neonatal leg muscles and four in leg muscles three days before birth. Regenerates of minced extensor digitorum longus or soleus muscles in adult animals had no native myosin the third day after surgery; they were similar to neonatal muscles 15 days after surgery and to adult muscles 60 days after surgery. Thus 
embryonic and neonatal myosin isozymes are transiently reexpressed in muscles regenerating in adult environment.

Bilateral changes in the parvalbumin content of muscles after unilateral cross-reinnervation in rats

M. Müntener, C. W. Heizmann and M. R. Celio

Anatomisches Institut und Institut für

Pharmakologie und Biochemie, Universität

Zürich-Irchel, 8057, Zürich, Switzerland

The $\mathrm{Ca}^{2+}$-binding protein parvalbumin (PV) presumably plays a role in the regulation of the contraction/relaxation cycle of the muscle fibre. Its content is high in fast (e.g. extensor digitorum longus, EDL) and low in slow (e.g. soleus, SOL) muscles.

To test the dependence of PV from the innervation, cross-reinnervation experiments between EDL and SOL were performed unilaterally. Three months later the muscles of both legs were investigated by histochemistry, immunohistochemistry, high performance liquid chromatography (HPLC) and radioimmunoassay (RIA) for their content of $\mathrm{PV}$. In a first experimental set the EDL muscle was cross-reinnervated by the SOL nerve $(X-E D L)$ and, as expected, it became slow and its PV content was correspondingly reduced. The $X-E D L$ receives in fact its motor input from the SOL motoneuron pool and its afferents reach the central nervous system via the SOL pathway. By interneuronal connections between the two SOL motoneuron pools it becomes 'neuronally' linked to the SOL of the contralateral side. Unexpectedly the contralateral SOL also shows a 'slowing down' with a reduced PV content. In a second set of experiments the SOL muscle was cross-reinnervated with the EDL (X-SOL). This led to a transformation of the $X$-SOL towards a fast muscle with an increased amount of PV. The 'neuronally' contralateral EDL muscle also exhibits an astonishingly massive increase of its PV content.

These pronounced contralateral changes may be the result of changes in central nervous activity and/or compensatory mechanical activity.

\section{Compensatory adaptation after selective denervation or lesions of skeletal muscle in a teleost fish \\ R. J. L. Pezenas, W. van Raamsdonk and \\ M. J. Smit-Onel \\ Department of Zoology, University of \\ Amsterdam, Kruislaan 320, 1098 SM Amsterdam, \\ The Netherlands}

Adaptive changes in the skeletal musculature of the zebrafish, Brachydanio rerio, induced by either partial denervation or lesions are being examined histochemically for possible use as markers preliminary to an investigation of the adaptive potential of motoneurons. Previous studies have indicated changes in metabolic markers (Wittenberger and Coprean, 1977) and morphological development (van Raamsdonk et al., 1982) after spinal cord lesion. In the present study, compensatory changes induced by selective denervation or lesion of slow red fibres on one side of the caudal peduncle are examined. An expected increase in the number of intermediate or FOG (fast oxidative glycolytic) fibres, which may be used for sustained swimming activity, at the expense of white or FG (fast glycolytic) 'burst swimming' fibres has not as yet been ascertained. However, combined atrophy and hyperplasia of red and intermediate fibres seems apparent in denervated fishes. This was observed especially on the operated side and extending, though to lesser degree, into the midbody region. Fishes in which red fibres were destroyed by lesion displayed atrophy and hyperplasia in the intermediate fibre region of the control side. Scattered hypertrophy of intermediate fibres in the treated area were also observed after both operations. An increase also appears in the number of scattered dorsal (SD) fibres, especially in the midbody region. Succinate dehydrogenase activity, as an indication of oxidative metabolism, decreases in denervated red muscle and in the midbody region cranial to denervation. Moreover, there are indications that some fibres in the intermediate area increase SDH activity. Such possible development of a mosaic of fibre types would have many ramifications for future considerations of adaptive interaction between neural and muscle cells.

\section{In vitro translation of mRNAs coding for citrate synthetase and lactate dehydrogenase isozyme 5 in chronically stimulated rabbit muscle}

U. Seedorf, E. Leberer and D. Pette Fakultät für Biologie, Universität Konstanz, Postfach 5560, D-7750 Konstanz, F.R.G.

Chronic, low frequency $(10 \mathrm{~Hz})$ stimulation induces in fast-twitch rabbit muscle a thorough rearrangement of the enzyme activity pattern of energy metabolism and a transition of the lactate dehydrogenase (LDH) isozyme pattern [Pette $e$ t al., Pflizgers Arch. 338, 257-72 (1973)]. Among 
others, there is an increase in mitochondrial enzyme activities of terminal substrate oxidation (e.g. the citric acid cycle) and an increase in the H-subunit of LDH [Klug et al., Pflügers Arch. 399, 280-4 (1983)]. The current study was undertaken in order to investigate possible relations between increases in citrate synthetase (CS) and LDH-5 and changes in transcriptional activity. Total activities of CS and LDH were determined in extracts from extensor digitorum muscles of rabbits stimulated ( $10 \mathrm{~Hz}, 12 \mathrm{~h} /$ day) from 2 to 60 days. The contribution of $\mathrm{H}$ - and $\mathrm{M}$-subunits to the total activity of LDH was assessed electrophoretically. Total mRNA was purified from the same muscles and translated in vitro using the reticulocyte lysate system. Translated CS and H-LDH proteins were immunoprecipitated and quantified after electrophoresis by measuring $\left.{ }^{35} \mathrm{~S}\right]$ methionine incorporation. The following results were obtained: CS activity increases slightly as soon as 2 days after the onset of stimulation. The main increase in this enzyme activity, however, is found between 6 and 14 days of stimulation. As judged from the amount of CS translated from total mRNA in vitro, a significant increase in mRNA coding for CS occurs only after stimulation periods of six days. The increase of in vitro translated CS is steep and reaches a plateau with an eight-fold increase after 10 days stimulation. It appears that the slight increase in CS activity preceding the rise in translatable mRNA is due to increased translation of pre-existing messenger, whereas the main increase in enzyme activity results from an increase both in transcription and translation. This assumption is supported by the findings on the $\mathrm{H}$-subunit of $\mathrm{LDH}$. An increase of $\mathrm{H}-\mathrm{LDH}$ in the stimulated muscle is found only after 6 days. Thereafter, H-LDH increases two-fold, reaching its maximum after 10 days stimulation. This time course corresponds to that of the increase in $\mathrm{H}-\mathrm{LDH}$ translated in vitro from total mRNA. The rise of H-LDH appears, therefore, to result primarily from increased transcription of the $\mathrm{H}$-LDH gene and concurrent translation of the messenger. The increase in mRNA is preceded by a rise in total RNA. Total RNA starts to increase linearly with time after two days, After two weeks, total RNA is at a threefold higher level in the stimulated than in the unstimulated muscle. At prolonged stimulation, total RNA decreases, and from three weeks on it stays at a two-fold elevated level. Since total RNA represents mainly ribosomal RNA, its early rise indicates an increased capacity for translation. (Supported by Deutsche Forschungs- gemeinschaft, Sonderforschungsbereich 138 and Sonderforschungsbereich 156.)

\section{Differentiation of regenerating muscle after denervation: an ultrastructural study}

S. Sesodia, M. J. Cullen and J. B. Harris

Muscular Dystrophy Research Laboratories,

Newcastle General Hospital, Westgate Road,

Newcastle upon Tyne, U.K.

Notexin, a myotoxin isolated from the venom of the Australian tiger snake causes a necrotic degeneration of skeletal muscle. The muscles regenerate and become virtually indistinguishable from normal by $2 I$ days after assault [Harris et al., Clin. exp. Pharmacol. Physiol. 2, 383-404 (1975); Harris \& Johnson, Clin. exp. Pharmacol. Physiol. 5, 587-600 (1978)]. Quantitative data on the assembly of contractile proteins are still lacking. In this investigation such data are presented and a comparison made with the regenerative response of denervated muscle.

Two micrograms of Notexin $(0.2 \mathrm{ml}$ of $10 \mu \mathrm{g}$ $\mathrm{ml}^{-1}$ in $0.9 \% \mathrm{NaCl}$ ) was injected into one hindlimb of female rats $(80-110 \mathrm{~g}$ body weight). In some animals, the limb was denervated just prior to the injection of toxin. Contralateral limbs served as controls. At fixed times after assault, soleus muscles were removed from both hindlimbs and processed for electron microscopical examination. In the injected limbs, the necrosis was complete between 24 and 36 hours. By 72 hours both innervated regenerating (IR) and denervated regenerating (DR) muscles contained myotubes, the mean diameter of which (\% of control fibres \pm 1 S.D.) was comparable $(30 \% \pm 3$ and $27 \% \pm 2$ respectively). The myotubes from IR and $\bar{D} R$ solei contained discrete bundles of myofilaments. During the following 11 days, small fibres formed in the IR solei which increased in diameter to become comparable with controls $(88 \% \pm 8)$. The ultrastructural organization of the myofilaments was indistinguishable from that in control fibres. In the DR solei, small fibres were also formed in the following 11 days and the ultrastructural organization of myofilaments of these developed to the same level as in the IR solei. These fibres, however, failed to increase in diameter reaching only $38 \% \pm 4$ of control. These results suggest that while the early development of muscle fibres in the DR solei is indistinguishable from that of those in IR solei, there is a marked reduction in subsequent growth. (The work was supported by a grant from the Medical Research Council of Great Britain.) 


\section{Is there a difference in fibre type distribution within a fascicle?}

M. Sjöström ${ }^{1}$, D. Y. Downham ${ }^{2}$ and J. Lexell ${ }^{1}$

${ }^{1}$ Departments of Neurology and Anatomy,

University of Umeå, Sweden

${ }^{2}$ Department of Statistics and Computational

Mathematics, University of Liverpool, U.K.

Analyses of cross-sections of whole human muscles, such as $\mathrm{m}$. vastus lateralis from young men, have shown that the proportion of fibres with different properties (type I 'slow-twitch', type 2 'fast-twitch') varies systematically with depth of the muscle [Lexell et al., J. Neurol. Sci. 61, 301-14 (1983)]. However, the arrangement of fibre types can be considered random as assessed by the number of enclosed fibres within smaller parts of the muscle [Lexell et al., J. Neurol. Sci. (in press)]. It was also noticed that the distribution of different fibres seemed to be uneven within single fascicles (Lexell et al., in press). The data in that second report have here been analysed in more detail. Furthermore, similar data from muscles of two groups of older men have been analysed to see whether or not the difference, if it exists, is a function of age. If there is a difference, i.e. a nonrandom element exists, then some hypotheses about factors determining muscle fibre properties become more plausible than others.

In every whole muscle cross-section, five areas were chosen; each $10-15 \mathrm{~mm}^{2}$. A total of 245 fascicles were analysed. For each fascicle the number of type $I$ and type 2 fibres, internally and on the boundary, were counted. A two-tailed sign test was used to test the (null) hypothesis that the proportions of type 1 fibres internal to, and on the boundary of, a fascicle were the same, against the (alt) hypothesis that these proportions were different. Wilcoxon's signed rank test was applied where the significance level in the sign test was greater than $1 \%$.

The results of the tests on each individual, each age group or each of five areas within the muscle were that the hypothesis of equal proportions was rejected at a highly significant level: the proportions of type 2 fibres on the boundaries were higher than internally. The difference was more marked in the young than in the old.

The immediate interpretation of our findings was that the different mechanical conditions for contraction between boundary and internal fibres, due to the characteristic arrangement of the connective tissue that surrounds the fascicle, influence the development of the fibre properties. The difference was unlikely to be the consequence of an altered afferent impulse-flow as the sensory system, e.g. the distribution of intrafusal fibres, is hardly so small-meshed. Only local factors then remain.

\section{Changes in the distribution of regulatory proteins in experimental disuse}

Ö. Takács and F. Guba

Institute of Biochemistry, University of Medical

Science, Szeged, Hungary

Earlier studies indicate that disuse has a marked effect on the biochemical and ultrastructural characteristics of skeletal muscles [Takács et al., Acta Biol. Acad. Sci. Hung. 32, 33-43 (1981); Guba et al. In Plasticity of Muscle (edited by Pette), pp. 507-21, Berlin: de Gruyter (1980)]. The myofibrillar proteins are involved either with the mechanics or the regulation of the contraction/ relaxation cycle. Considering the latter function, it was reasonable to investigate, whether the regulatory proteins resemble the transformation of myosin subunit distribution in experimental disuse. Immobilization of the right hindlegs of adult rabbits was performed as described earlier (Takács et al., 1981), holding the gastrocnemius and soleus muscles in shortened position. After 2-4 weeks the affected and corresponding control muscles were excised and the myofibrillar fraction was prepared. The material was used either for analysis of the protein patterns on $I$ and $2 \mathrm{D}$ gels, or for isolation of regulatory proteins. The results of $\mathrm{I}$ and $2 \mathrm{D}$ gels reveal that the relative distribution of tropomyosin and troponin subunits change as a result of disuse. 2D electrophoresis of isolated troponin has provided valuable new information concerning the heterogeneity of this protein showing that $\mathrm{Tn}-\mathrm{T}$ appears in eight spots having different isoelectric points, while Tn-I gives 3-4 spots. In Tn-T isolated from immobilized soleus a minimum of 12 spots could be detected indicating the synthesis of fast troponin, i.e. the partial slow to fast transformation of the muscle cells. Multiple isoelectric variants of $\mathrm{Tn}-\mathrm{T}$ and $\mathrm{Tn}-\mathrm{I}$ in 2D maps believed to be due to presence of phosphorylated proteins. The results suggest that the changes in the type of myosin light chains (Takács et al, 1981) and regulatory protein subunits induced by immobilization may be a matter of adaptation which takes place by translational or post-translational modification of the type-specific proteins. 


\section{ENERGETICS AND METABOLISM}

\section{Parallel changes in contractile properties and myofibrillar ATPase activity of rat myocardium during postnatal growth \\ V. Cappelli, C. Reggiani, C. Poggesi and \\ R. Bottinelli \\ Istituto di Fisiologia umana, Università di Pavia, \\ Pavia, Italy}

In order to study developmental changes of the contractile material of mammalian heart, myofibrillar ATPase activity and force-velocity relationship were determined in left ventricular myocardium from 10- (newborn), 20-, 60- (young) and 240(adult) day-old rats. Myofibrils from the free wall of the left ventricle were prepared and purified according to the method developed by Solaro et al. [Biochim. Biophys. Acta 245, 259 (1971)]. Proteins were measured by the method of Lowry; inorganic phosphate was measured with the Fiske-Subarow method. Conditions of assay for ATPase were: $2 \mathrm{~mm}$ ATP, $2 \mathrm{~mm} \mathrm{MgCl}, 50 \mathrm{mM} \mathrm{KCl}, 20 \mathrm{~mm}$ imidazole, $\mathrm{pH} 7.0,0.5-2.0 \mathrm{mg}$ protein, $2 \mathrm{~min}$, $26^{\circ} \mathrm{C}, 10 \mathrm{ml}$ final volume.

Significant changes of ATPase activity were found to occur during postnatal growth. The activity increased from $0.186 \pm 0.007 \mu \mathrm{M} \mathrm{P}_{\mathrm{i}} / \mathrm{mg}$ protein $/ \mathrm{min}(n=6)$ at 10 days to $0.264 \pm 0.024$ $(n=6)$ at 20 days. Further increase in age was accompanied by a slight but progressive decrease in the enzymatic activity; this was $0.255 \pm 0.009$ $(n=15)$ at 60 days and $0.230 \pm 0.009(n=10)$ at 240 days. Same results were obtained with the same buffer added with EGTA $1.6 \mathrm{mM}$ and $\mathrm{Ca}^{2+}$ $1 \mathrm{~mm}$. Furthermore the ATPase activity of the different age groups exhibited a similar $\mathrm{Ca}^{2+}$ sensitivity in the 8-4 pCa range.

Force-velocity relationships were determined from afterloaded contractions in papillary muscles isolated from the left ventricle $\left(26^{\circ} \mathrm{C}\right.$, optimal length, stimulation rate $2 / \mathrm{min}$ ). The velocity of shortening measured at a relative load of $0.3 P_{\text {o }}$ showed the same age-related modifications as described for the myofibrillar ATPase activity. Shortening velocity increased (ca. $40 \%$ ) from 10 to 20 days and then progressively decreased by approximately the same extent from 20 to 240 days. The mechanical changes observed are likely to be linked to the modifications of the enzymatic activity of the contractile proteins. In addition a possible role of postnatal changes in the activation level reached by myocardial cells in our experimental conditions should be taken into account. In fact, the low stimulation rate utilized was chosen in order to obtain the highest mechanical output from cardiac specimens of adult rats. However a positive staircase phenomenon and a great sensitivity to inotropic interventions can be observed in neonatal rat myocardium. This suggests that in the experimental conditions utilized a much lower level of activation is reached by the neonates than by the adults.

\section{Activation of actomyosin ATPase activity by filamin}

Renata Dabrowska, A. Goch, Hanna Osińska,

A. Szpacenko and J. Sosińsk.i

Nencki Institute of Experimental Biology,

3 Pasteur Str., 02-093 Warsaw, Poland

It has been shown previously that thin filaments from chicken gizzard muscle activate the $\mathrm{Mg}^{2+}$-ATPase activity of skeletal muscle myosin to a greater extent than does the complex of gizzard actin and tropomyosin [Szpacenko et al., J. Musc. Res. Cell Motility 5, 228 (1984)]. The protein factor responsible for this additional activation was identified as a high molecular weight actin binding protein, filamin.

Activation of actomyosin ATPase activity by filamin takes place with molar ratios of filamin to actin of $1: 200$ to $1: 30$. Within these ratios a loose meshwork of actin filaments and a gelation of actin solution by filamin occurs. Treatment of the F-actinfilamin complex with cytochalasin $\mathrm{B}\left(\mathrm{IO}^{-4} \mathrm{M}\right)$ abolishes activation. Activation is also destroyed by the $\mathrm{Ca}^{2+}$-dependent protease which is known to degrade filamin to heavy merofilamin (a fragment that binds to actin but does not cause crosslinking of actin filaments). Maximum activation of actomyosin ATPase activity by filamin takes place at $30-65 \mathrm{mM} \mathrm{KCl}$, at $\mathrm{pH} 6.5$ and $30^{\circ} \mathrm{C}$.

In the presence of tropomyosin activation of actomyosin ATPase requires more filamin than that required in the absence of tropomyosin. Filamin does not activate ATPase activity of acto-subfragment- 1 and has only a slight effect on $\mathrm{Mg}^{2+}$-ATPase of acto-heavy meromyosin. This latter observation, as well as the lack of activation at $0^{\circ} \mathrm{C}$ and in $100 \mathrm{mM} \mathrm{KCl}$ suggest that the activation of actomyosin ATPase by filamin is due to its effect on the structure of the superprecipitating actomyosin gel. 


\section{Energy metabolism in muscles of frogs intoxicated with an isomer of creatine, $\boldsymbol{\beta}$-guanidinopropionate}

M. De Saedeleer, J. Lebacq and G. Maréchal

Department of Physiology, University of Lowvain, B-1200 Brussels, Belgium

Summer frogs (Rana temporaria) were injected every two days through the mouth into the ventral lymphatic space with $60 \mathrm{mg}$ of $\beta$-guanidinopropionate dissolved in $1 \mathrm{ml}$ of $127 \mathrm{mM} \mathrm{NaCl}$ for 45 days. They were kept at room temperature in running tap water at $14^{\circ} \mathrm{C}$. Control frogs were kept in separate boxes. Sartorius muscles were isolated and poisoned with $0.4 \mathrm{~mm}$ iodo-acetate. They were stimulated at $20^{\circ} \mathrm{C}$ in nitrogen at length $l_{0}$ for either 2,4 or $8 \mathrm{~s}$. The force developed was recorded isometrically. Stimuli were supramaximal condenser discharges of alternating polarity at $50 \mathrm{~Hz}$. The muscles used for chemical determinations were frozen on the last shock of the tetanus by rapid immersion in isopentane cooled to $-160^{\circ} \mathrm{C}$ with liquid nitrogen, and then extracted with perchloric acid. Creatine and $\beta$-guanidinopropionate, both free and total, were measured by colorimetric method. ATP, ADP and AMP were measured enzymatically. In parallel experiments heat production was measured in separate muscles using a Hill-Downing type thermopile. In comparison with sartorius muscles of untreated frogs: muscles poisoned with $\beta$-guanidinopropionate contained $\beta$-guanidinopropionate, both free and phosphorylated. They contained more free creatine, less phosphocreatine and the same amount of total creatine; the concentration of ATP was not changed significantly. Phosphorylated $\beta$-guanidinopropionate was not split during an isometric tetanus. The expenditure of chemical energy was computed as the sum of phosphocreatine breakdown and twice the ATP breakdown. In all muscles examined in heat experiments the cumulated production was proportional to the tension-time integral; but muscles from poisoned frogs produced significantly more heat than those from unpoisoned ones. No significant difference in maintenance of tension was observed between the two groups of muscles. In unpoisoned muscles, the apparent enthalpy change of $\sim P$ hydrolysis was $44.2 \mathrm{~kJ} \mathrm{~mol}^{-1}$ throughout the tetanus. In muscles poisoned with $\beta$-guanidinopropionate the enthalpy change increased progressively from $40.3 \mathrm{~kJ} \mathrm{~mol}^{-1}$ after $2 \mathrm{~s}$ of tetanus to $68.8 \mathrm{~kJ} \mathrm{~mol}^{-1}$ after $8 \mathrm{~s}$.
Effects of catecholamines on some mechanical properties of rat soleus muscle in hyperthyroidism

M. E. Everts, W. S. Simonides and

C. van Hardeveld

Department of Chemical Pathology, University

Hospital of Leiden, The Netherlands

Catecholamines (CA) and thyroid hormones are important regulators of the mechanical properties of skeletal muscle. The observation that betaantagonists may improve muscle strength in thyrotoxic patients [Lancet ii, 219 (1968)], also suggests a possible involvement of CA in muscle functioning in hyperthyroidism. The purpose of the present study was to examine the effects of propranolol (PRO) and isoproterenol (ISO) on force and relaxation rate (RR) during $10 \mathrm{~Hz}$ stimulation in soleus muscle of euthyroid $(C)$ and hyperthyroid $\left(T_{3}\right)$ rats. In addition, we determined the formation of phosphorylase a (Ppa) because this process is under control of the adrenergic system. Force at $10 \mathrm{~Hz}(\mathrm{~N})$ was significantly lower in the $\mathrm{T}_{3}$ rats $\left[C(n=8): 0.52 \pm 0.03 ; \mathrm{T}_{3}(n=7): 0.33 \pm 0.01\right.$, $P<0.001$ (mean \pm S.E.M.)], whereas RR (\% force loss $/ 10 \mathrm{~ms})$ was twice as high $[\mathrm{C}(n=8)$ : $\left.11.1 \pm 0.8 ; \mathrm{T}_{3}(n=7): 20.3 \pm 1.0, P<0.001\right]$. The changes in force, $R R$ and $P p a$ produced by PRO and ISO and the number of beta-receptors are summarized in the table $\left({ }^{*} P<0.05\right.$ or better).

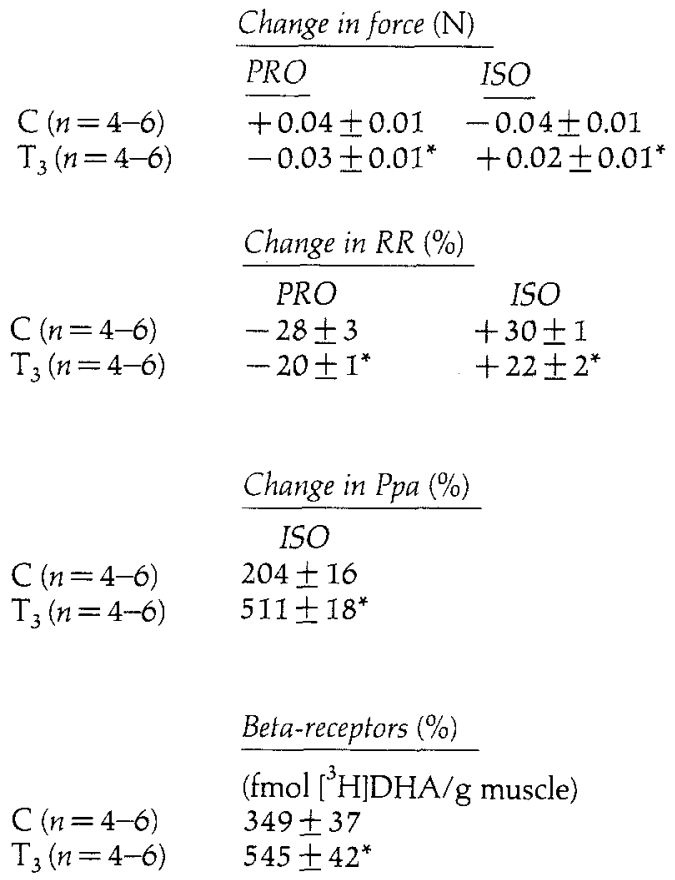


As shown above, PRO could not restore force and $R R$ in the $T_{3}$ rats to the level in the $C$ rats. The higher number of beta-receptors in the $T_{3}$ rats correlated well with the greater effect of ISO on $\mathrm{Pp} a$, but not with the mechanical observations. Moreover, the ISO-produced increase in Ppa was completely abolished by PRO in both groups, whereas the increase in $R R$ was only partially blocked. The suggestion that stimulation of the $\mathrm{Ca}^{2+}$ release from the sarcoplasmic reticulum (SR) may also contribute to the mechanical effects of CA [J. Physiol. 315, 267 (1981)], was confirmed in our study by the finding that ISO could reduce the inhibition of force by dantrolene (an inhibitor of the $\mathrm{Ca}^{2+}$ release from the SR). The $\mathrm{Ca}^{2+}$-loading capacity of the SR $\left(\mu \mathrm{mol} \mathrm{Ca} \mathrm{Ca}^{2+} / \mathrm{g} / 20 \mathrm{~min}\right)$ was increased two-fold in the $\mathrm{T}_{3}$ rats [C $(n=7): 42.0 \pm$ $\left.0.6 ; \mathrm{T}_{3}(n=7): 76.0 \pm 1.0, P<0.001\right]$ indicating a much larger capacity for $\mathrm{Ca}^{2+}$ sequestration and $\mathrm{Ca}^{2+}$ release in this group. In conclusion, our results do not point to a role for CA in the thyroidhormone induced changes in force and $R R$ in rat soleus muscle. We suggest that differences in intracellular $\mathrm{Ca}^{2+}$-cycling between $\mathrm{C}$ and $\mathrm{T}_{3}$ rats may contribute to the observed different mechanical responses to ISO and PRO.

\section{Energetics and ultrastructure in skeletal and heart muscle \\ H. Hoppeler, K. Conley, K. Rösler and \\ K. Schwerzmann \\ Department of Anatomy, University of Berne, \\ CH-3012, Bern, Switzerland}

The maximal peak rate of energy consumption of muscle tissue is set by the ATPase activity of myosin and the sarcoplasmic reticulum. The maximal steady-state energy consumption seems to be limited at a much lower level, by the rate at which oxygen is delivered to muscles as well as by the oxidative potential of their mitochondria. Skeletal muscle mitochondria may vary considerably in their capacity to use various substrates for oxidative phosphorylation. It is well established that chronic electrical stimulation and endurance exercise increases their capacity for fatty acid oxidation severalfold. On the other hand mitochondria seem to be highly conservative structures, with regard to the enzymes involved in the energy transduction, i.e. the Krebs cycle, the respiratory chain and ATP synthesis. Most of these enzymes are 'densely' packed into the inner mitochondrial membrane and it is found that the surface density of these membranes remain constant with endurance exercise, chronic electrical stimulation and among animals of different body mass (approx. $30 \mathrm{~m}^{2}$ $\mathrm{cm}^{-3}$ ). The oxidative potential of muscles would therefore be regulated by quantitative changes of its mitochondrial content, rather than by qualitative changes within the mitochondria themselves. In various experimental models it is found that skeletal muscle mitochondria consume $\mathrm{O}_{2}$ at an almost constant rate of $4-6 \mathrm{ml}, \mathrm{O}_{2} \mathrm{~min}^{-1} \mathrm{~cm}^{-3}$. In heart tissue preliminary results suggest that the aerobic power production of a unit volume of heart tissue is related to the volume density of mitochondria, whereas the power output of the whole heart is proportional to the total volume of mitochondria.

\section{Effects of creatine phosphate and its} hydrolysis products on $\mathrm{Ca}^{2+}$-regulated force production in skinned cardiac muscles J. C. Kentish

Department of Physiology, University College

London, Gower Street, London WCIE 6BT, U.K.

When cardiac muscle is made hypoxic, developed tension falls to a low level within a few minutes. This early contractile failure is accompanied by changes in $\mathrm{pH}$ and by the hydrolysis of the 15-30 mM creatine phosphate (CP) in the cytosol to orthophosphate $\left(\mathrm{P}_{\mathrm{i}}\right)$ and creatine $(\mathrm{Cr})$ [Allen t al., J. Physiol., Lond. 343, 58-9P (1983)]. It seemed possible that these changes in the concentrations of $\mathrm{CP}$ and its products could, by direct actions on the contractile machinery, at least partly account for the early contractile failure. To test this, the effects of $\mathrm{CP}, \mathrm{P}_{i}$ or $\mathrm{Cr}$ on myofibrillar force production were investigated using detergent-skinned trabeculae from rat hearts. Control solutions contained $5 \mathrm{~mm}$ ATP and $10 \mathrm{mM} \mathrm{CP}$ at $\mathrm{pH} 7.0$. As reported by Herzig et al. [Biochim. Biophys. Acta 672, 191-6 (1981)], the maximum force (at $\left.\left[\mathrm{Ca}^{2+}\right]>100 \mu \mathrm{M}\right)$ was strongly inhibited by $\mathrm{P}_{\mathrm{i}}$. Maximum force (at constant [ATP] and [CP]) was reduced by up to $50 \%$ in $5 \mathrm{mMP}_{i}$ and by up to $80 \%$ in $30 \mathrm{mM} \mathrm{P}$. This effect was not due to a dysfunction of the ATP-regenerating system as a result of an inhibition of creatine kinase activity [Nihei $e t$ al., J. Biol. Chem. 236, 3203-9 (1961)] because it was also seen if the $10 \mathrm{~mm} \mathrm{CP}$ in the control solutions was replaced by ATP; neither was it simply a consequence of the decrease in the affinity for ATP hydrolysis [cf. Dawson et al., ]. Physiol., Lond. 299, 465-84 (1980)], since the addition of ADP, which would also reduce this affinity, did not alter maximum force production. In addition to its effect on maximum force, $\mathrm{P}_{\mathrm{i}}$ shifted the force $-\left[\mathrm{Ca}^{2+}\right]$ 
relationship to higher $\mathrm{Ca}^{2+}$ concentrations, i.e. the $\mathrm{Ca}^{2+}$-sensitivity of the myofibrils was decreased.

An increase in CP from $10 \mathrm{~mm}$ (control) to $30 \mathrm{~mm}$ lowered maximum force by $20-30 \%$, although $\mathrm{CP}$ did not appear to alter the $\mathrm{Ca}^{2+}$-sensitivity. $\mathrm{Cr}$ had no effect on force production.

The combined inhibitory effects of an elevated $\left[\mathrm{P}_{\mathrm{i}}\right]$ on maximum force and on $\mathrm{Ca}^{2+}$-sensitivity were such that they could contribute substantially to the early contractile failure produced by hypoxia in living cardiac muscle, although their net action would be partly offset by the reduction in the inhibitory effect of $\mathrm{CP}$ as the $[\mathrm{CP}]$ fell. (Supported by a grant from the B.H.F.)

\section{Phosphorylase $a$ formation, myosin light chain phosphorylation and the energy turnover in contracting skeletal muscle of hypothyroid rats \\ W. J. Leijendekker and C. van Hardeveld Department of Chemical Pathology, University Hospital, Leiden, The Netherlands}

Thyroid hormones have been shown to influence energy metabolism in skeletal muscle during muscular contraction. Recently we showed that under conditions of maximal stimulation force development proceeds more economically in hypothyroid (Tx) rats [Leijendekker et al., Metabolism 32, 615-21 (1983)]. In order to find an explanation for this phenomenon the effect of thyroid-hormone depletion on the energy generating process under anaerobic conditions was investigated. For that reason the formation of phosphorylase $a(\% \mathrm{Pp} a)$ and the energy turnover were determined at different times during tetanic stimulation of the gastrocnemius-plantar muscle. The total phosphorylase activity was the same for $T x$ and euthyroid (C) rats. The formation of Ppa after $5 \mathrm{~s}$ of stimulation was identical for $\mathrm{C}$ and $\mathrm{Tx}$ rats $[\%$ Ppa: $59 \pm 4(\mathrm{C}, n=5) ; 55 \pm 4(\mathrm{Tx}, n=6)( \pm$ S.D.)]. Between 5 and $10 \mathrm{~s}$ of stimulation the \% $\mathrm{Ppa}$ in the muscles of $\mathrm{Tx}$ rats decreased with $50 \%$ but remained unchanged in the $C$ group. The energy cost after $5 \mathrm{~s}$ of stimulation was somewhat but not significantly lower in the Tx group [C $(n=5)$ : $0.54 \pm 0.04 \mu \mathrm{mol} \sim \mathrm{P} / \mathrm{N} \mathrm{s}$; Tx $(n=5): 0.46 \pm 0.04]$. In the 5-10s time interval of stimulation, the energy cost for contraction decreased markedly in the Tx group $[0.22 \pm 0.05(n=9)]$ but only slightly in the $C$ group $[0.46 \pm 0.14(n=6)]$. Since a correlation has been observed between a decrease in the energy cost for contraction and an increase in the degree of myosin light chain $\left(\mathrm{m}-\mathrm{LC}_{2}\right)$ phosphorylation [Crow \& Kushmerick, Science 217,
835-7 (1982)], also the extent of $\mathrm{m}-\mathrm{LC}_{2}$ phosphorylation was measured. The percentage of $\mathrm{m}-\mathrm{LC}_{2}$ phosphorylation after $5 \mathrm{~s}$ (C: $78 \%$ : Tx: $54 \%$ ) and after 10 s (C: $82 \%$; $\mathrm{T}$ : 61\%) was significantly higher in the $\mathrm{C}$ group.

In conclusion, the decrease in energy turnover during tetanic stimulation in the hypothyroid group correlates well with a decrease in Ppa formation, but does not correlate with the degree of $\mathrm{m}-\mathrm{LC}_{2}$ phosphorylation.

\section{The effect of energy depletion with a creatine analogue on $\mathrm{E}-\mathrm{C}$ coupling after a tetanus} G. W. Mainwood

Department of Physiology, University of Ottawa, Ottawa K1H 8M5, Ontario, Canada

Rats were fed on a diet containing the creatine analogue $\beta$-guanidinopropionate, for four weeks. The skeletal muscles of these rats were depleted of creatine, creatine phosphate and ATP so that total energy reserves $(\mathrm{ATP}+\mathrm{CrP})$ were less than $20 \%$ of normal. Contractile responses of these muscles to a single stimulus of a brief train of stimuli were not significantly different from normal. The posttetanic responses of the EDL muscles in situ were measured following a Is $100 \mathrm{~Hz}$ stimulus train.

The post-tetanic potentiation $(+80 \%)$ seen in the normal muscles is reversed in the energy depleted muscles. The suppression is short lasting and the response returns to normal $1-2 \mathrm{~s}$ after the tetanus $(-40 \%)$. This is followed by a period of reduced potentiation $(+20 \%)$. The potentiating effect of the tetanus on the contractile response of normal muscles is progressively reduced as the number of stimuli used to elicit the test response is increased. In energy depleted muscles the posttetanic depression is progressively reduced by increasing the number of stimuli. The maximum developed force to a brief train of stimuli $(400 \mathrm{~Hz}$, $50 \mathrm{~ms}$ ) given after a tetanus is very similar in normal and depleted muscles. In both types of muscle the amplitude of the surface electrical response is not significantly different.

It seems probable that in energy-depleted muscles some energetic parameter such as $\Delta G_{\text {ATP }}$ is reduced below a critical level as a result of the tetanus. This critical level is essential for adequate $\mathrm{E}-\mathrm{C}$ coupling. Although some energy-independent parameter facilitates $\mathrm{E}-\mathrm{C}$ coupling in normal muscles under these conditions this effect is overcome for at least $1-2 \mathrm{~s}$ by the decreased energetic parameter. (This work was supported by the Canadian Medical Research Council.) 
$\mathrm{Ca}^{2+}$-calmodulin modulation of muscle phosphofructokinase

Georg W. Mayr

Institut für Physiologische Chemie I, Ruhr

Universität, D-4630 Bochum, G.F.R.

Muscle phosphofructokinase (PFK) has been identified as a calmodulin (CM) binding protein [Mayr \& Heilmayer, FEBS Lett. 159, 51-7 (1983)]. Binding studies, enzymatic, light scattering and sedimentation analyses were performed in order to elucidate the role of the $\mathrm{Ca}^{2+}-\mathrm{CM}$ interaction with PFK [Mayr, Eur. J. Biochem. (in press)]. The binding of CM to PFK is strictly $\mathrm{Ca}^{2+}$-dependent $\left(K_{\mathrm{app}} \sim 1 \mu \mathrm{M}\right)$. The apparent binding affinity and stoichiometry depend on the association state of the enzyme. PFK previously dissociated to dimers binds $2 \mathrm{~mol} \mathrm{CM}$ per mol enzyme protomer $\left(M_{\mathrm{r}}\right.$ 86000 subunit), one with high affinity $\left(K_{\text {app }}\right.$ $<50 \mathrm{nM})$, one with lower affinity $\left(K_{\mathrm{app}} \sim\right.$ I $\left.\mu \mathrm{M}\right)$. In tetrameric PFK only one class of binding sites with low affinity was identified. CM can enhance the dissociation of active tetramers into the inactive dimers at physiological enzyme concentrations and metabolic conditions which without $\mathrm{CM}$ would prevent dissociation. A prerequisite is a low degree of occupancy of effector binding sites on PFK which if saturated stabilize the tetrameric state. These are the hexosebisphosphate- and the regulatory ATP-binding sites. AMP acts also antagonistic to $\mathrm{CM}$ if bound in presence of hexose bisphosphate, otherwise it acts synergistically with CM. Metabolic conditions allowing for this inactivation are met at low contractile activity. CM binding to dimeric PFK slowly induces a conformational change which prevents a rapid reassociation into active tetramers upon CM dissociation. These conformers tend to form large inactive filamentous polymers. The polymerization reaction is enhanced at $1: 1$ binding stoichiometry but can be suppressed by a saturation of the second CM-binding site. Most drastically the polymerization proceeds if after a prolonged incubation with $2 \mathrm{~mol} \mathrm{CM}$ per mol protomer $\mathrm{CM}$ is dissociated by lowering $\mathrm{Ca}^{2+}$. Polymers formed slowly can depolymerize if $\mathrm{Ca}^{2+}-\mathrm{CM}$ is reassociated. Only in this unpolymerized form the conformational change can be slowly reversed by a saturation of the regulatory ATP-binding sites. Thus a reactivation of PFK previously inactivated again requires CM. Metabolic conditions favouring this reaction are met at high contractile activity. $\mathrm{Ca}^{2+}-\mathrm{CM}$ thus may act as a catalyst of a slow redistribution of PFK between an active and an inactive pool, the direction depending on actual metabolic conditions. In the allosteri- cally regulated tetrameric enzyme fraction, $\mathrm{CM}$ binding also antagonizes the binding of ATP to the regulatory sites. Consequently the allosteric inhibitory action of ATP is weakened and the catalytic activity increases instantaneously. Besides the long-term effects, $\mathrm{CM}$ thus may also induce a rapid, $\mathrm{Ca}^{2+}$-triggered activation of glycolysis in muscle.

The control of the asynchronous flight motor in a beetle (Melolontha melolontha)

Peter Schneider

Biologie für Mediziner, Universität

Heidelberg, G.F.R.

In insects we find two types of flight muscles: (1) direct or synchronous muscles with a 1 : I relation between nervous excitation and muscle contraction, (2) indirect or asynchronous muscles with no 1: 1 relation. In beetles it could be shown that there is no direct relationship between wing beat (representing the contraction) and nervous control. Spikes can be found in each phase before, during or after the contractions.

In sections of long flights we could not find a rhythmical or serial phase in the progress of the interspikes. Starts and flights have different interspike patterns, even in the same individual. Muscles with the same function $\left(\mathrm{DVM}_{1-3}, \mathrm{ODM}\right.$, 8 upstroke muscles with different directions for contraction) have no co-ordination between the different motoneurons. Each muscle has one or more motoneurons, which excitate the corresponding muscle separately. The same can be said for antagonistic muscles. No relation could be found between flight control (directional change or flight speed) and excitation pattern of flight muscles. Once started the flight motor oscillates, all flight manoeuvres occur by small direct muscles.

Based on general muscle physiology and experiments with isolated muscles, we suggest that the action potentials of muscles with the same function or with antagonistic functions are responsible only for the regulation of $\mathrm{Ca}$. If the $\mathrm{Ca}$-level is at an optimal concentration (start $=$ initial burst of action potentials), a tension by the antagonistic muscles is sufficient to release the contraction. For the maintenance of the oscillation a certain rate of contractions per interspike should not be understepped; if this happens the flight motor runs to an end within a few strokes. 
The tracheal supply of the indirect flight muscles in an insect (Melolontha, Coleoptera) P. Schneider ${ }^{1}$, B. Hoese ${ }^{1}$ and H. H. Janssen ${ }^{2}$ ${ }^{1}$ Biologie für Mediziner, and ${ }^{2}$ Zoologisches Institut, Universität Heidelberg, G.F.R.

Entering the indirect flight muscle the tracheal system indents the sarcolemma to the end. The finest terminal tracheoles (diameter $200-300 \mathrm{~nm}$ ) are also surrounded by sarcolemma. The typical endcell (Wigglesworth, 1930), which is perforated by the terminal tracheoles, does not exist in the flight muscles of beetles. The tracheoblast cells, which create tracheae and tracheoles, also accompany the tracheoles to the end. The ramification of the tracheoles occurs in the tracheoblast. This cell system is filled with many mitochondria (sarcosomes) and small fat droplets. The T-system, which is responsible for the distribution of the depolarization, originates once at the sarcolemma outside the muscle cell and twice from the indented sarcolemma of the tracheae and tracheoles.

Three functions of the tracheal system in flight muscles can be discussed: (1) respiration; (2) maintenance of the metabolism during long distance flight with fat; and (3) electromechanical coupling.

\section{Role of myofibrillar creatine kinase on relaxation of rigor tension in cardiac muscle R. Ventura-Clapier and G. Vassort Laboratoire de Physiologie Cellulaire Cardiaque, U-241 INSERM, Université Paris XI, 91405 Orsay, \\ France}

Cardiac myofibrils contain bound MM-creatine kinase (MM-CK) which is able to rephosphorylate $\mathrm{ADP}$ at the expense of creatine phosphate $(\mathrm{CP})$ in the cytosol. A tight enzymatic coupling has been shown in isolated myofibrils between MM-CK and myosin ATPase [Saks et al., Biochim. Biophys. Acta
803, 254-64 (1984)]. In this study we investigated the functional significance of this coupling. Experiments were performed on chemically (Triton $\mathrm{X}-100)$ skinned right papillary muscles of rat at very low free calcium concentration $\left(10^{-9} \mathrm{M}\right)$ and various ATP, ADP and $\mathrm{CP}$ concentrations. The medium contained $30 \mathrm{~mm}$ imidazole buffer, $10 \mathrm{~mm}$ EGTA, $30 \mathrm{mM} \mathrm{Na}^{+}, \mathrm{pMg}^{2+} 2.5$, at $\mathrm{pH} 7.1$ and $22^{\circ} \mathrm{C}$; ionic strength was adjusted to $0.16 \mathrm{~mm}$ by $\mathrm{K}$ acetate. In the absence of $\mathrm{CP}$, relaxation of rigor tension by $\mathrm{MgATP}$ was obtained with half maximal effect for $1.8 \mathrm{~mm} \mathrm{MgATP}$. In the presence of $12 \mathrm{mM} \mathrm{CP}$ and $250 \mu \mathrm{M}$ ADP, decreasing MgATP concentration even to $10^{-9} \mathrm{M}$ never induced rigor tension. At very low MgATP concentration $\left(10^{-6} \mathrm{M}\right)$, the relaxation of rigor tension was dependent upon ADP and CP concentrations. Halfmaximal relaxing effect was obtained with $2 \mathrm{~mm}$ $C P$, a value close to the $K_{\mathrm{m}}$ of isolated MM-CK for this substrate, or with $14 \mu \mathrm{M}$ ADP, a value five times lower than the reported $K_{m}$. These functional aspects reinforce the proposal of a tight coupling between myosin ATPase and MM-CK. An exogenous ATP regenerating system (phosphoenol pyruvate + pyruvate kinase) was not able to fully relax the fibres. When MM-CK was inhibited by FDNB, the dependency of rigor tension towards $\mathrm{MgATP}$ became the same as it was without CP. The addition of exogenous MM-CK for half an hour fully relaxed rigor tension; however, this effect persists even after prolonged washout (half an hour) as if added MM-CK was able to rebind to the myofibrils and to produce the same effect as native MM-CK. These results show that endogenous MM-CK is able to ensure maximal efficiency of myosin ATPase by producing a localized high ATP concentration; they also suggest the existence of rapidly exchangeable binding sites for MM-CK in cardiac myofibrils.

\section{CALCIUM-BINDING PROTEINS AND MEMBRANES}

Inhibition of phosphorylase kinase by antibodies against the delta-subunit (calmodulin)

H. Böhm and H. P. Jennissen

Institut für Physiologie. Physiologische Chemie

and Ernährungsphysiologie der Universität

München, Veterinärstrasse 13, D-8000

München 22, F.R.G.

Monospecific antibodies were generated against the purified delta-subunit of phosphorylase kinase [Jennissen et al., Hoppe-Seyler's Z. Physiol. Chem. 360, 293 (1979)] and purified by affinity chromatography on calmodulin Sepharose. The antibodies were quantified by RIA [Van Eldik \& Watterson, J. biol. Chem. 256, 4205-10 (1981)] ELISA in the system described in Böhm et al. [J Immun. Meth. 70, 193-209 (1984)] and characterized by inhibition assays (see Böhm et al., 1984). 
In an inhibition assay at $\mathrm{pH} 6.8$ containing about $2.5 \mu \mathrm{g}$ nonactivated phosphorylase kinase $/ \mathrm{ml}$ the enzyme activity was inhibited $50 \%\left(K_{0.5}^{i}\right.$ by about $22 \mu \mathrm{g}$ anti-delta/ml. Maximal inhibition was about $80 \%$. At pH 8.2 employing $0.25 \mu \mathrm{g} \mathrm{ml} \mathrm{m}^{-1}$ phosphorylase kinase a $50 \%$ inhibition was found with about $100 \mu \mathrm{g} \mathrm{ml}-1$ anti-delta. Contrary to the behaviour of monospecific antibodies against the other three subunits of phosphorylase kinase [Jennissen et al., J. Musc. Res. Cell Motility 6, 96 (1985)], the ratio of anti-delta protein to phosphorylase kinase in the assay for $50 \%$ inhibition is about 45 -fold lower at $\mathrm{pH} 6.8$ indicating that phosphorylase kinase is much more sensitive to inhibition at this $\mathrm{pH}$ and might be exposing calmodulin in a different conformation than at $\mathrm{pH}$ 8.2.

At $\mathrm{pH} 6.8$ phosphorylase kinase ( $1.25 \mu \mathrm{g} \mathrm{ml}^{-1}$ ) is activated [for assay see Jennissen \& Heilmeyer, Analyt. Biochem. 57, 118-26 (1974)] about fivefold by exogenous purified delta subunit (i.e. delta'). The degree of activation $\left(\varepsilon_{a}\right)$ is a hyperbolic function of the calmodulin concentration $\left(\varepsilon_{\mathrm{a}, \max }=\right.$ 5.5; $K_{0.5}^{a}=49 \mathrm{~mm}$ ). In four-fold activated enzyme (delta: delta $=1: 19) \varepsilon_{a}$ can be fully reduced from 3 to 0 by anti-delta $\left(K_{0.5}^{\mathrm{i}}=22 \mu \mathrm{g}\right.$ anti-delta $\left./ \mathrm{ml}\right)$. This corresponds to a maximal inhibition of $70-80 \%$ if the total activity of the activated enzyme is taken as $100 \% \quad\left(K_{0.5}^{i}=35 \mu \mathrm{g}\right.$ antidelta $/ \mathrm{ml})$. Since the integral calmodulin subunit of phosphorylase kinase is purely regulatory and not catalytically active it appears that the antibody is reversing the conformational change of phosphorylase kinase induced by $\mathrm{Ca}^{2+}$ or exogenous $\mathrm{Ca}^{2+}$-calmodulin.

\section{Studies on conformational changes induced by} $\mathrm{Ca}^{2+}$ and actin in gelsolin

A. Fattoum, J. F. Rouayrenc, C. Mejean and

R. Kassab

C.R.B.M., CNRS Route de Mende, 34033

Montpellier Cedex, France

Gelsolin is a $92000 \mathrm{Ca}^{2+}$ binding protein which reversibly shortens actin filaments. Gelsolin is present in nonmuscle cells as well as in smooth, skeletal and cardiac muscles [Yin et al., J. Cell Biol. 91, 901-6 (1981)]. We purified to homogeneity a 92000 polypeptide from rabbit and dog heart muscle. This protein was found to be similar if not identical to nonmuscle cell gelsolin. At micromolar $\mathrm{Ca}^{2+}$ concentration, muscle gelsolin accelerated $\mathrm{G}$-actin polymerization. It also binds to and severs preformed actin filaments. Tropomyosins protect actin filaments against severing [Fattoum et al.,
Biochemistry 22, 1187-93 (1983)]. Analysis of cardiac muscle extracts obtained at high and low ionic strength showed that this protein is tightly bound to myofibrils. Indirect immunofluorescence microscopy revealed that as in skeletal muscle, gelsolin shares the same position as actin in cardiac muscle [Rouayrevic et al., FEBS Lett. 167, 52-8 (1984)].

The conformational changes associated with $\mathrm{Ca}^{2+}$ and F-actin binding to gelsolin (from plasma) could be monitored by limited digestion with trypsin. In EGTA, gelsolin was only slightly attacked by the protease, but $\mathrm{Ca}^{2+}$ induced a typical fragmentation pattern of the protein whereas $\mathrm{Mg}^{2+}$ was without effect. The interaction with $\mathrm{F}$-actin (molar ratio $1: 2$ ) led to a specific splitting of gelsolin both in the absence and presence of $\mathrm{Ca}^{2+}$. Studies on the nature and function of the gelsolin fragments are underway.

\section{Phosphorylase kinase: a multifunctional Ca-calmodulin regulated protein-lipid-kinase} Z. Georgoussi ${ }^{1}$, Z. Hessova, J. W. Crabb, M. Varsanyi and L. M. G. Heilmeyer Jr. ${ }^{2}$

IThe National Hellenic Research Foundation, 48, Vassileos Constantinou Ave., Athens

501/1.,Greece

${ }^{2}$ Institut für Physiologische Chemi I,

Ruhr-Universität, Universitätsstraße 150,

4630 Bochum, F.R.G.

Phosphorylase kinase accepts several proteins as substrate and expresses three partial activities called $A_{0}, A_{1}$ and $A_{2}$ [Kilimann \& Heilmeyer, Biochemistry 21, 1727-35 (1982)]. Additionally, this enzyme phosphorylates phosphatidylinositol which is associated with the $\mathrm{Ca}^{2+}$ transport ATPase of fast skeletal muscle sarcoplasmic reticulum [Varsanyi \& Heilmeyer, EMBO ]. 1543-8 (1983)]. This report describes the dual function of calmodulin in phosphorylase kinase to regulate differentially the $\mathrm{Ca}^{2+}$-independent $A_{0}$ activity from the $\mathrm{Ca}^{2+}$-dependent activities $A_{1}$ and $A_{2}$. Furthermore it describes the relationship of phosphorylase kinase and phosphatidylinositol kinase.

The $\mathrm{Ca}^{2+}$-independent $A_{0}$ activity can be reversibly stimulated by heparin more than 20-fold. Concomitantly, the $\mathrm{Ca}^{2+}$-dependent $A_{2}$ activity is abolished completely. Structural studies show that calmodulin dissociates from phosphorylase kinase under these conditions. It suggests that $\mathrm{Ca}^{2+}$-free calmodulin inhibits the $\mathrm{Ca}^{2+}$-independent activity $A_{0}$, whereas $\mathrm{Ca}^{2+}$ saturation of the $\mathrm{Ca}^{2+} / \mathrm{Mg}^{2+}$ high affinity sites stimulates the activity $A_{1}$ and $\mathrm{Ca}^{2+}$ saturation of the $\mathrm{Mg}^{2+}$-inducible $\mathrm{Ca}^{2+}$ low affinity sites stimulates the activity $A_{2}$. 
Following the phosphatidylinositol kinase activity during a standard phosphorylase kinase purification shows that both activities are enriched approximately to the same degree. Diacylglycerol kinase activity is separated. Neither chromatography on DEAE cellulose nor on hydroxyapatite in the presence of $I \mathrm{M} \mathrm{KCl}$ separates phosphatidylinositol kinase from phosphorylase kinase.

The first 17 residues of the $\mathrm{N}$-terminal sequence of the phosphorylase kinase $a$ subunit exhibits six identities and one conservative interchange with the pp $60^{v-s r c}$ tyrosine kinase [Crabb \& Heilmayer, J. biol. Chem. 259, 6346-50 (1984)]. Very recently, this tyrosine kinase was shown also to phosphorylate phosphatidylinositol and diacylglycerol [Sugimoto et al, Proc, natn. Acad. Sci. U.S.A. 81, 2117-221 (1984)]. Consequently, the phosphatidylinositol kinase but not the diacylglycerol kinase activity in the phosphorylase kinase might be associated with the $a$ subunit of the enzyme.

\section{Calcium binding proteins from eel muscle C. Gerday, J.-M. Francois and I. Dubois Laboratoire de Biochimie Musculaire, Institut de Chimie, Sart Tilman, 4000 Liege, Belgium}

The calcium-binding proteins, troponin-C, calmodulin and the parvalbumins were isolated from eel (Anguilla anguilla) muscle. Three parvalbumins isotypes (V, III, II) were purified to homogeneity. Their overall concentration in the muscle is $0.5 \mathrm{mmol} \mathrm{kg}{ }^{-1}$ wet weight; all contain one residue of tyrosine and their physico-chemical properties are typical of this family of proteins. The troponin- $\mathrm{C}$ was isolated from an acetone powder extracted at high ionic strength, followed by separation of the troponin subunits by ionexchange chromatography in $6 \mathrm{M}$ urea and final purification on Sephacryl-S200. This protein is devoid of tyrosine and contains only one single tryptophane residue. It therefore shows an unusual ultraviolet absorption spectrum and its fluorescence properties are strongly affected by the presence of $\mathrm{Ca}^{2+}$, since the cation induced a $20 \mathrm{~nm}$ shift of the fluorescence spectrum towards shorter wavelengths associated with a large increase of the fluorescence intensity. Calmodulin was extracted in the presence of ethylene diamine tetraacetate (EDTA) followed by trichloroacetic acid fractionation, and separation on DEAE cellulose and Aca54. Troponin-C free calmodulin $(0.1 \mu \mathrm{mol}$ per $\mathrm{kg}$ wet weight) was obtained by FPLC chromatography on a Mono $Q$ column.
Some functional properties of caldesmon

A. Goch, Barbara Gałazkiewicz, Hanna Osińska and Renata Dabrowska

Nencki Institute of Experimental Biology,

3 Pasteur Str., 02-093 Warsaw, Poland

Caldesmon, a calmodulin and actin-binding protein composed of the two polypeptide chains of molecular weights 150000 and 147000 , was discovered in smooth muscle of chicken gizzard by Sobue et al. [Proc, natn. Acad. Sci. U.S.A. 78, 5652-5 (1981)]. Since caldesmon with calmodulin affects a superprecipitation of actomyosin gel in a $\mathrm{Ca}^{2+}$-dependent manner, its role in regulation of smooth muscle contraction has been proposed [Kakiuchi \& Sobue, Trends Biochem. Sci. 8, 59-62 (1983)].

We have studied the interaction of caldesmon with F-actin and its effect on ATPase activity of skeletal muscle actomyosin. We have shown that caldesmon causes parallel alignment of actin filaments into bundles. In the presence of calmodulin and the absence of $\mathrm{Ca}^{2+}$ nearly all actin filaments are arranged into bundles, whereas in the presence of $\mathrm{Ca}^{2+}$ only a few bundles surrounded by a loose meshwork of actin filaments are observed.

Interaction of caldesmon with $\mathrm{F}$-actin reflects on the interaction of the latter protein with myosin. Under the conditions when bundles of actin filaments are formed, ATPase activity of actomyosin is inhibited. Caldesmon alone inhibits actomyosin ATPase to maximum $30 \%$; in the presence of smooth muscle tropomyosin the inhibition is potentiated up to $60-70 \%$. Substitution of myosin by either heavy meromyosin or subfragment-I does not affect the inhibition of ATPase activity by caldesmon.

Caldesmon induces polymerization of G-actin at low ionic strength solution. This effect is inhibited by calmodulin in the presence of $\mathrm{Ca}^{2+}$.

\section{Parvalbumin in muscle and nonmuscle cells}

C. W. Heizmann

Institut für Pharmakologie und Biochemie,

Universität Zürich-Irchel, $\mathrm{CH}-8057$ Zürich,

Switzerland

Parvalbumin, together with calmodulin, S-100 proteins, troponin- $C$ and the vitamin $\mathrm{D}$-dependent $\mathrm{Ca}^{2+}$-binding proteins $(\mathrm{CaBP})$, osteocalcin, skin $\mathrm{CaBP}$ and oncomodulin, belongs to a family of homologeous $\mathrm{Ca}^{2+}$-binding proteins. Although these proteins are structurally related, their cellular and intracellular distribution is quite different, indicating distinct physiological functions.

Applying immunohistochemical techniques, 
parvalbumin was found exclusively in type II (fasttwitch) mammalian skeletal muscle fibres which could be further subdivided into five subgroups displaying distinct staining intensities. Cardiac muscle fibres and smooth muscle cells were never labelled by the parvalbumin antiserum. A quantitative analysis of various muscle extracts for parvalbumin by high performance liquid chromatography on reverse-phase supports showed a direct correlation between parvalbumin concentration and half-relaxation time, suggesting together with some kinetic measurements that parvalbumin has a role to play in the relaxation process.

Parvalbumins are, however, not restricted to contractile cells but are also found in a few other tissues e.g. in a restricted population of neurons in the central nervous system and in specific cells in endocrine glands (the Leydig cells of the testis) and this suggests an involvement in the regulation of processes other than those specific for fast-twitch muscles.

More recently, three cell-lines [LICR, (Lond)$\mathrm{HN} 1,-\mathrm{HN} 2$, and -HN6] derived from human carcinomas and displaying varying degrees of locomotive activity were investigated for the presence of parvalbumin. Applying two different immunohistochemical methods in conjunction with a monospecific anti-parvalbumin serum an intense staining was observed in cells displaying translocative motility. Often, in these cells an association with as yet undefined net-like structures in the nuclear region was observed.

These tumour cells contained a novel protein structurally and immunologically related to parvalbumin which was absent from comparable normal tissues. In analogy to muscle, we suggest that this protein might be tumour-associated and connected to the motile behaviour of these carcinoma cells.

\section{Activation of phosphorylase kinase by antibodies against the beta- and gamma-subunits}

H. P. Jennissen, G. Botzet and

J. K. H. Petersen-von Gehr

Institut für Physiologie, Physiologische Chemie and Ernährungsphysiologie der Universität München, Veterinärstr. 13, D-8000 München 22,

F.R.G.

Monospecific antibodies were generated against the alpha-, beta- and gamma-subunits (called anti- $a$, anti- $b$ and anti-g) of phosphorylase kinase Uennissen et al., Hoppe-Seyler's Z. Physiol. Chem. 360, 293 (1979), Böhm et al., J. Immun. Meth.
70, 193-209 (1984)] and characterized by ELISA, double immunodiffusion, quantitative immunoprecipitation and microcomplement fixation (Böhm et al., 1984). These antisera contain inhibiting (Jennissen et al., 1979; Böhm et al., 1984) and activating antibodies [Jennison et al., 1979; Jennison et al., J. Musc. Res. Cell Motility 1, 210 (1980)]. Activation of phosphorylase kinase by antibodies is only found under certain conditions. Thus the $\mathrm{Ca}^{2+}$-independent activity [for definition see Kilimann \& Heilmeyer, Biochemistry 21, 1727-34 (1982)] can only be inhibited and not activated by anti- $a$, anti- $b$ and anti-g both at pH 6.8 and 8.2. Depending on the immunization schedule, anti- $a$, anti- $b$ and anti-g also inhibit the $\mathrm{Ca}^{2+}$ dependent enzyme activities at both $\mathrm{pH}$ values. The ratio of antibody to enzyme protein necessary for half-maximal inhibition at $\mathrm{pH} 6.8$ is equal to or larger than at $\mathrm{pH} 8.2$. Kinetically (in respect to phosphorylase $b$ ) inhibition appears to be due to a mixed type of mechanism. Certain antiserum fractions of anti- $b$ and anti-g activate the $\mathrm{Ca}^{2+}$. dependent activity 3-4-fold only at $\mathrm{pH}$ 6.8. This activation, which is not additive if the two antisera are mixed, is strongly dependent on the $\mathrm{pH}$ 6.8/8.2 activity ratio of the enzyme and disappears at high ratios e.g. 0.08-0.1. Kinetically (in respect to phosphorylase $b$ ) activation is the result of a 5-10-fold increase in $V_{\max }$ and not a decrease in $K_{\mathrm{m}}$.

As a steric hindrance of substrate accessibility to the enzyme by antibody can only lead to an inhibition of activity, the mechanism of activation can be attributed to antibody-induced conformational changes. Since the inhibition of phosphorylase kinase at $\mathrm{pH} 6.8$ by anti- $a$ can be fully reversed by the addition of activating anti- $b$, it is concluded that the mechanism of inhibition of phosphorylase kinase, e.g. by anti-a is also largely due to antibody-induced conformational changes and not steric hindrance.

\section{Benzocaine, a neutral local anaesthetic, inhibits calcium release from the sarcoplasmic reticulum}

T. J. Lea

University Laboratory of Physiology, Parks Road, Oxford OX1 3PT, U.K.

Benzocaine is a neutral local anaesthetic $\left(\mathrm{p} K_{\mathrm{a}}=2.6\right)$ and a structural analogue of procaine, lacking procaine's ionizable diethylamine group $\left(\mathrm{p} K_{\mathrm{a}}=8.9\right)$. Thus at physiological $\mathrm{pH}, 7.1$, benzocaine is uncharged whereas procaine is $98 \%$ cationic. The effects of benzocaine on $\mathrm{Ca}$ release from the sarcoplasmic reticulum (SR) of barnacle muscle 
(Balanus nubilus) were compared with those of procaine, a known inhibitor of Ca release. Isometric tension responses of myofibrillar bundles were used to assess $\mathrm{Ca}$ release at $\mathrm{pH}$ 7.1. Dimethylsulphoxide (DMSO) was used to prepare a stock solution of benzocaine; therefore $1 \%$ DMSO was included in all bathing solutions. Contractures which were induced by $5 \mathrm{~mm}$ caffeine were inhibited by more than $90 \%$ by both benzocaine and procaine $(1-5 \mathrm{~mm})$. Carbon dioxide-induced Ca release was obtained by replacing $\mathrm{Cl}^{-}$in the bathing solution with $\mathrm{HCO}_{3}$ and bubbling with $100 \% \mathrm{CO}_{2}$, adjusting the $\mathrm{pH}$ to 7.1 . Benzocaine, like procaine, virtually abolished the $\mathrm{CO}_{2}$-induced response. The presence of $1 \%$ DMSO reduced the amplitude of control $\mathrm{CO}_{2}$ responses by about $30 \%$. Effects of the anaesthetics on Ca-activation of the contractile proteins were tested by first exposing the myofibrillar bundles to solutions containing $0.5 \%$ Brij $58,1 \%$ Triton $X-100$ and 2 mM EGTA for $45 \mathrm{~min}$. This treatment abolished both the responses to caffeine and $\mathrm{CO}_{2}$, presumably through the breakdown of the SR membranes. Contractures which were then elicited by the addition of $\mathrm{CaCl}_{2}$ to the normal bathing solution ( $+1 \%$ DMSO) were not inhibited by more than $10 \%$ by $1-5 \mathrm{~mm}$ benzocaine.

It is concluded that benzocaine can inhibit $\mathrm{Ca}$ release from the SR of barnacle muscle as effectively as procaine and that the ionizable group of the procaine molecule $\left(p K_{a}=8.9\right)$ is not necessary for procaine's action. This result does not support the contention that it is the cation of amine anaesthetics which is the active inhibitory form at the SR [Bianchi \& Bolton, ]. Pharmacol. exp. Ther. 157, 388-405 (1967); Seeman, Pharmacol. Revs, 24, 583-655]. Neither does it support the idea that procaine prevents calcium release by acting as a weak base and increasing the $\mathrm{pH}$ within the $\mathrm{SR}$ lumen [Lea, J. Physiol, Lond. 330, 52-3P], since the benzocaine molecule is unable to accept protons at physiological $\mathrm{pH}$ values and consequently cannot function as a weak base. (Supported by the M.R.C.)

\section{Calcium binding and structural characteristics or frog muscle calsequestrin}

S. Salvatori, E. Damiani and A. Margreth

N.R.C. Unit for Muscle Biology and

Physiopathology, Institute of General Pathology.

via Loredan 16, 35100 Padova, Italy

Skeletal muscle calsequestrin (CS) is a SR intraluminal $\mathrm{Ca}^{2}+$-binding protein that has been well characterized, mainly in the rabbit, for its peculiar, electrophoretic properties in two-dimensional SDSgels according to Michalak et al. [J. biol. Chem. 255, 1317 (1980)] and its staining characteristics with the carbocyanine dye Stains All. Frog muscle calsequestrin is slightly more acidic and its apparent molecular weight ( $M_{\mathrm{r}}: 73000$ in Laemmli's system) and maximal $\mathrm{Ca}^{2+}$-binding capacity $\left(66 \mathrm{~mol} \mathrm{Ca}^{2+}\right.$ $\mathrm{mol}^{-1}$ ), as determined by equilibrium dialysis in 5 mM Tris-- $\mathrm{HCl}, \mathrm{pH} 7.5$, according to MacLennan \& Wong [Proc. natn. Acad. Sci. U.S.A. 68, 1231 (1971)] appear to be distinctly higher than has been reported for rabbit CS. One-dimensional peptide mapping after partial digestion with $S$. aureus proteinase did not reveal any obvious structural difference. However amino acid analysis of frog muscle CS demonstrated a similar content of acidic aminoacid residues, but a relatively higher percentage of aspartic acid versus glutamic acid $(28 \%$ and $10 \%$ respectively), as compared to other species [MacLennan \& Wong, 1971; White et al., Biochim. Biophys. Acta, 744, 1 (1983)]. Furthermore, we found that the carbocyanine dye Stains All interacts with frog muscle CS forming a different complex that absorbs maximally at $540 \mathrm{~nm}$ ( $\beta$ state) and which corresponds to a different complex state (J-state) from that found with rabbit CS, having an absorbtion maximum at $600 \mathrm{~nm}$ [Campbell et al, J. biol. Chem. 258, 1126 (1983)]. A $\beta$-spectral band upon reaction with Stains All is characteristic of macromolecules having closely spaced anionic groups, such as polyaspartic acid [Kay et al., ]. Phys. Chem. 68, 1896 (1964)]. Our results suggest that the observed differences in $\mathrm{Ca}^{2+}$-binding capacity between frog and rabbit CS are related to differences in the composition of anionic sites within the molecule. (Work supported by institutional funds from C.N.R. and a grant from the Dino Ferrari Foundation.)

\section{Calcium uptake by sarcoplasmic reticulum prepared from the asynchronous flight muscles of Phormia terrae-novae}

R. Semich and H. Volmer

Zoologisches Institut, University of Münster,

Hindenburgplatz 55, D-4400 Münster, F.R.G.

Sarcoplasmic reticulum (SR) vesicles were purified from the asynchronous flight muscles of the blowfly Phormia terrae-novae by cell fractionation and sucrose gradient centrifugation. In these SR preparations the calcium pump protein $\left(M_{\mathrm{r}}=110000\right)$ was the dominating component accounting for approximately $65 \%$ of the total protein. The calcium uptake rate of these $S R$ preparations was $4.4 \mu \mathrm{mol} \mathrm{min}^{-1}$ and $\mathrm{mg}\left(25^{\circ} \mathrm{C}\right)$. The specific 
calcium uptake rate amounted to $6.6 \mu \mathrm{mol} \mathrm{mg}^{-1}$ $110000 M_{\mathrm{r}}$ protein in $1 \mathrm{~min}$. This value is similar to that found in SR preparations of synchronous insect muscles (locust flight muscles, cockroach leg muscles).

In order to quantify total uptake differences in different types of muscles the calcium uptake rate in homogenates of several synchronous and asynchronous insect flight muscles has been determined. It was found that, in general, total calcium uptake activity in asynchronous flight muscles

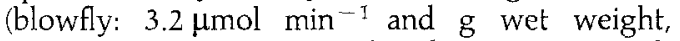
honeybee: $2.3 \mu \mathrm{mol} \mathrm{min}{ }^{-1}$ and $\mathrm{g}$ ww) is much lower than in synchronous muscles (locust leg muscles: 41.9 , locust flight muscles: 41.7 , cockroach leg muscles: $49.7 \mu \mathrm{mol} \mathrm{min}^{-1}$ and $\mathrm{g} w \mathrm{w}$ ).

The reduction of calcium uptake activity in asynchronous flight muscles could be, in principle, explained (1) by a diminution of the SR volume and/or by (2) a reduction of the transport activity of the calcium pump protein of the SR. The latter possibility, however, can be excluded since the specific calcium uptake rate in Phormia flight muscle SR was not lower than in SR preparations of various synchronous insect muscles. Therefore the reduction of total calcium uptake activity in the asynchronous flight muscles of Phormia terraenovae is probably due to the reduction of SR volume which has earlier been demonstrated in this type of muscle [Smith, Sci. Am. 212 (1965)].

\section{Is there a correlation between parvalbumin content and myosin light chain $\mathrm{LC} 2_{\mathrm{f}}$ phosphorylation? \\ I. Stuhlfauth and H. Jockusch \\ Development Biology Unit, W7, University of \\ Bielefeld, POB 86 40, D-4800 Bielefeld 1, F.R.G.}

We have studied polypeptide patterns in the skeletal muscle of mouse mutants that are affected in their neuromuscular system [Peters, Mouse News Lett. 70, 4-44 (1984)]. A striking reduction of the calcium-binding protein, parvalbumin (PV), was observed in the mutant 'arrested development of righting response', adr [Stuhlfauth et al., Proc. natn. Acad. Sci. U.S.A. 81, 4814-18 (1984)]. In the same mutant, the fraction of phosphorylated fast myosin light chain $2\left(\mathrm{LC}_{\mathrm{f}} \mathrm{P} / \mathrm{LC} 2_{\mathrm{f}}+\mathrm{LC} 2_{\mathrm{f}} \mathrm{P}\right)$ in the tibialis anterior muscle was reduced to 0.14 in comparison to a wild type level of 0.48 [cf. Crow \& Kushmerick, J. biol. Chem. 257, 212I-4 (1982)]. Since the ATPase fibre patterns (IIA, IIB fast fibres) were retained, the polypeptide changes are not a consequence of the disappearance of IIB fibres. In two other mutants, wobbler (wr) and motor end- plate disease (med, med ${ }^{j o}$ ) neither $\mathrm{PV}$ nor $\mathrm{LC} 2_{\mathrm{f}} \mathrm{P}$ were drastically reduced in comparison to wildtype. This apparent parallel behaviour of PV and LC $2_{\mathrm{f}}-\mathrm{P}$ is being further investigated by comparing different developmental stages in addition to different genotypes in the mouse. A possible link between PV content and the degree of $\mathrm{LC}_{2}$ phosphorylation could be the $\mathrm{Ca}^{2+}$ regulation in the muscle fibre [cf. Moss, J. Musc. Res. Cell Motility 3, 295-311 (1982)]. (This work is supported by Deutsche Forschungsgemeinschaft, grant DFG Jo $84 / 7$.

\section{Calmodulin-dependent phosphorylation and dephosphorylation of cardiac sarcoplasmic reticulum}

J. Suko, B. Plank, C. Pifl, W. Wyskovsky and

G. Hellmann

Institute of Pharmacology, University of Vienna,

Austria

Calmodulin (CaM)-dependent stimulation of calcium (Ca) transport by cardiac sarcoplasmic reticulum (SR) has been suggested to be due to phosphorylation of phospholamban and/or subunits by a $\mathrm{Ca}$, CaM-dependent protein kinase [Le Peuch et al., Biochemistry 18, 5150 (1979)]. In previous studies a positive correlation was obtained between CaM-dependent increase in the rate of $\mathrm{Ca}$ transport and hydroxylamine-insensitive phosphoester which is formed by the $\mathrm{Ca}$, CaM-regulated protein kinase, indicating a causal relationship between both processes [Plank et al., Eur. ]. Biochem. 136, 215 (1983)].

The aim of our investigations on the effect of CaM on cardiac SR was to identify the CaM-Ca species which activate the $\mathrm{Ca}, \mathrm{CaM}$-dependent, membrane-bound protein kinase responsible for CaM-dependent phosphorylation of $\mathrm{SR}$ and to determine whether the $\mathrm{Ca}$, CaM-dependently formed phosphoesters are dephosphorylated by an endogenous phosphatase present in the SR fraction.

Activation of CaM-dependent phosphorylation by free $\mathrm{Ca}(0.2-10 \mu \mathrm{M})$ and total CaM concentrations ranging from 0.01 to $I \mu \mathrm{M}$ gave apparent $\mathrm{K}(\mathrm{Ca})$ from $2.49-0.62 \mu \mathrm{M}$ and Hill coefficients of about 4 . A single activation curve could be fitted to these phosphoprotein values depending on the concentration of the calculated $\mathrm{CaM} \cdot\left(\mathrm{Ca}^{2+}\right)_{4}$ species (apparent $K_{d} 0.89 \mathrm{nM}$ ) which was in good agreement with the apparent dissociation constant of $2.5 \mathrm{nM}$ of high affinity CaM binding determined by $\left({ }^{125} \mathrm{I}\right) \mathrm{CaM}$ binding to SR fractions at $\mathrm{I} \mathrm{mM} \mathrm{Ca}$. These data indicate that $\mathrm{CaM} \cdot\left(\mathrm{Ca}^{2+}\right)_{4}$ is the only 
active $\mathrm{CaM}-\mathrm{Ca}$ species which regulates the $\mathrm{Ca}$, CaM-dependent protein kinase of cardiac SR [Pifl et al., J. Biochim. Biophys. Acta 773, 187 (1984)]. The Ca, CaM-dependently formed phosphoesters of SR are dephosphorylated by a phosphoprotein phosphatase present in the SR fractions, which is slightly stimulated by $\mathrm{Mn}$ and strongly inhibited by $\mathrm{NaF}$ or orthophosphate. The rate of dephosphorylation and inhibition are similar to the dephosphorylation of phospholamban prior phosphorylated by an exogenous cAMP-dependent protein kinase.

\section{Immunological and biochemical evidence of a phosphatidylinositol-phosphorylase-kinase in SR-membranes}

R. Thieleczek ${ }^{1}$, G. Behle ${ }^{1}$, L. M. G. Heilmeyer $\mathrm{Jr}^{1}$, Z. Hessová ${ }^{1}$, M. Varsanyi ${ }^{\mathrm{I}}$ and D. Drenckhahn ${ }^{2}$

${ }^{1}$ Institut für Physiologische Chemie I,

Ruhr-Universität, Universitätsstraße 150,

4630 Bochum, G.F.R.

${ }^{2}$ Institut für Anatomie und Zellbiologie,

Philipps-Universität, 3550 Marburg, G.F.R.

Monoclonal antibodies specific for the $\alpha_{-}, \beta$ - and $\gamma$-subunits of rabbit fast skeletal muscle phosphorylase kinase ( $\mathrm{PlaK}$ ) were used to determine the subcellular location of these subunits in rabbit psoas major muscle. In frozen sections of acetoneand formaldehyde/glutaraldehyde-fixed muscle, incubation with these antibodies resulted in a diffuse cytoplasmic staining pattern which was most prominent in the intermyofibrillar and perinuclear space. The pattern correlated well with the cytosolic origin of the holoenzyme. In sections of unfixed muscle extensively rinsed with phosphatebuffered saline prior to immunostaining, the diffuse cytoplasmic immunofluorescence was largely reduced and a staining pattern reminiscent of the cellular distribution of the sarcoplasmic reticulum (SR) was visible. Dotted staining was apparent at the level of the Z-lines and I-bands of longitudinally cut fibres (cross-sectioned terminal cisternae) as well as honeycomb-like patterns in crosssectioned fibres (SR surrounding the myofibrils). These staining patterns were especially prominent for the anti- $a$ antibody and less evident for the other two. For immunostaining at the ultrastructural level polyclonal antibodies to the entire holo-PlaK complex $(\alpha, \beta, \gamma, \delta)_{4}$ were used. Immunoperoxidase label was found at two cellular sites: (I) diffusely scattered in the interfibrillar space and some I-bands and (2) along the membranes of the SR. In addition the polyclonal antibodies cross-reacted with the basal lamina. These observations indicate that PlaK or a part of it, the $a$-subunit, may be associated with both a cytosolic component (i.e. glycogen) and the SR-membrane. Recent data indicate that PlaK also contains a soluble phosphatidylinositol kinase (PinK) activity which is probably inherent in the $a$-subunit [Varsanyi et al., EMBO J. 2, 1543-8 (1983); Georgoussi \& Heilmeyer, unpublished; Crabb \& Heilmeyer, J. biol. Chem. 259, 6346-50 (1984); Sugimoto et al., Proc. natn. Acad. Sci. U.S.A. 81, 2117-21 (1984)]. Thus, it is reasonable to assume that the PinK associated with the SR-membrane is related, if not identical, to the $a$-subunit of PlaK.

A SR-membrane-bound PlaK activity could be separated by Triton X-114 and transferred to the water phase to a level of about $40 \%$. By this treatment a (PinK) activity is always coextracted to the same extent. These two activities cannot yet be separated.

\section{Calmodulin-dependent protein phosphorylation and $\mathrm{Ca}^{2+}$-permeability in sarcoplasmic reticulum vesicles from skeletal muscle \\ B. S. Tuana, V. K. Khanna and D. H. MacLennan}

Banting and Best Department of Medical Research, University of Toronto, Toronto, M5G 1L6,

Canada

When sarcoplasmic reticulum vesicles were extracted with EGTA or EDTA an increase in membrane permeability resulted in a decrease in $\mathrm{Ca}^{2+}$ accumulation and stimulation of $\mathrm{Ca}^{2+}$-dependent ATPase activity. The addition of the extract, in the presence of $\mathrm{Ca}^{2+}, \mathrm{Mg}^{2+}$ and ATP, enhanced $\mathrm{Ca}^{2+}$ accumulation by chelator-extracted sarcoplasmic reticulum vesicles. Calmodulin was found to substitute for the extract in the reconstitution reaction and calmodulin antagonists inhibited the calmodulin-dependent reconstitution of $\mathrm{Ca}^{2+}$ uptake. The amount of calmodulin associated with the sarcoplasmic reticulum membrane was found to be about $0.2 \mu \mathrm{g} \mathrm{mg}^{-1}$ of protein, $90 \%$ of which was released by EGTA or EDTA extraction. Exogenous calmodulin in the presence of $\mathrm{Ca}^{2+}$, $\mathrm{Mg}^{2+}$ and ATP also enhanced $\mathrm{Ca}^{2+}$ accumulation by unextracted sarcoplasmic reticulum vesicles and by sarcoplasmic reticulum vesicles extracted at alkaline $\mathrm{pH}$. A calmodulin-dependent protein kinase, intrinsic to the membrane, was found to be activated under the conditions of reconstitution, resulting in enhanced incorporation of ${ }^{32} \mathrm{P}$ from ATP into proteins of $M_{r} 85000,60000,22000$ and 20000 .

These results suggest a role for the calmodulin- 
dependent phosphorylation system in coupling $\mathrm{Ca}^{2+}$ accumulation and $\mathrm{Ca}^{2+}$-ATPase activity, perhaps through regulation of $\mathrm{Ca}^{2+}$ release channels in sarcoplasmic reticulum vesicles. The calmodulin-induced increase in $\mathrm{Ca}^{2+}$ accumulation may result from decreased $\mathrm{Ca}^{2+}$ permeability due to the closure of $\mathrm{Ca}^{2+}$ release channels through protein phosphorylation.

\section{Degradation of phosphatidylinositolphosphate in SR membranes}

M. Varsanyi ${ }^{1}$, M. Schäfer ${ }^{2}$, H. M. Assy ${ }^{2}$ and

L. M. G. Heilmeyer ${ }^{1}$

${ }^{1}$ Institut für Physiologische Chemie I,

Ruhr-Universität, Universitätsstraße 150,

4630 Bochum, G.F.R.

${ }^{2}$ Department of Soit and Agricultural Chemistry,

Faculty of Agriculture, Mansoura University.

Mansoura, Egypt

Phosphatidylinositolphosphate (DPI) is formed in isolated $\mathrm{Ca}^{2}+$ transport ATPase from fast skeletal muscle sarcoplasmic reticulum (SR) when this protein is incubated with $\mathrm{ATP} / \mathrm{Mg}^{2+}$ in the absence and presence of exogeneously added phosphatidylinositol kinase (EC 2.7.1.67) at $1.6 \mathrm{nM}$ free $\mathrm{Ca}^{2+}$. After reaching a steady-state phosphorylation level, enhancement of the free $\mathrm{Ca}^{2+}$ for about $0.5 \mu \mathrm{M}$ causes a rapid decomposition of DPI. Until now no experimental proof exists for the participation of a $\mathrm{Ca}^{2+}$-dependent phosphatidylinositol-4 phosphate phosphodiesterase in this process. Removing either ATP or $\mathrm{Mg}^{2+}$ from the phosphorylation mixture without addition of $\mathrm{Ca}^{2+}$ results also in DPI decomposition. However, $20 \mathrm{~mm}$ sodium fluoride completely inhibits the $\mathrm{Ca}^{2+}$-dependent disappearance of DPI. Fluoride does not remove $\mathrm{Ca}^{2+}$ under these conditions since a $\mathrm{Ca}^{2+}$-stimulated $\mathrm{Ca}^{2+}$-transport ATPase activity can be measured. Consequently, an enzyme may be responsible for the DPI dephosphorylation which is inhibitable by fluoride. Most likely, it is a phosphomonoesterase which is independent of $\mathrm{Ca}^{2+}, \mathrm{Mg}^{2+}$ or $\mathrm{ATP} / \mathrm{Mg}^{2+}$, at the micromolar level.

The $\mathrm{Ca}^{2+}$ transport ATPase containing radioactively labelled DPI was reisolated. With this material it can be shown that the DPI decomposition is not influenced by $\mathrm{Ca}^{2+}$ from $0.05 \mu \mathrm{M}$ to $10 \mu \mathrm{M}$ or calcium/calmodulin. From these experiments we conclude that $\mathrm{Ca}^{2+}$-stimulated DPI decomposition is due to a phosphatidylinositol kinase inhibition rather than a $\mathrm{Ca}^{2+}$-accelerated decomposition.

\section{The effect of various phospholipids on the thermostability of sarcoplasmic reticulum adenosine triphosphatase from locust flight muscles and crayfish abdominal muscles \\ D. Veltel and H. Volmer}

University of Münster, Zoological Institute,

Hindenburgplatz 55, D-4400 Münster, F.R.G.

Sarcoplasmic reticulum (SR) $\mathrm{Ca}^{2+}$-ATPase from crayfish (Astacus leptodactylus) abdominal muscles shows a much lower thermostability than the ATPase from locust (Locusta migratoria) flight muscle SR [Volmer, Comp. Biochem. Physiol. 70A, 351-8 (1981)]. In order to test the extent to which the thermostability can be changed by various phospholipids, the $\mathrm{Ca}^{2+}$-ATPase from both crayfish and locust SR was delipidated and reactivated by exogenous phospholipids with fatty acids differing in the chain length (dimyristoyllecithin, DML, 14:0; 14:0; dipalmitoyllecithin, DPL, $16: 0 ; 16: 0$ and distearoyllecithin, DSL, 18:0; 18:0) and the degree of unsaturation (dioleoyllecithin, DOL, 18:1; 18:1 and dilinoleoyllecithin, DLL, $18: 2 ; 18: 2$ ).

The $\mathrm{Ca}^{2+}$-ATPase from locust SR whose phospholipid moiety was predominantly replaced by DSL, DPL and DOL showed a temperature optimum in the $46-50^{\circ} \mathrm{C}$ range, i.e. the thermostability was comparatively high. The temperature optimum of the DML-ATPase and the DLL-ATPase was considerably lower $\left(40^{\circ} \mathrm{C}\right.$ and $35^{\circ} \mathrm{C}$, respectively). Similar effects could be observed when the $\mathrm{Ca}^{2+}$-ATPase from crayfish SR was delipidated and reactivated by the phospholipids named above. The temperature optima ranged from $30^{\circ} \mathrm{C}$ (DLL-ATPase) to $42^{\circ} \mathrm{C}$ (DSL-ATPase).

The results show that phospholipids and, in particular, the chain length and the degree of unsaturation of the fatty acids of these phospholipids have a profound influence on the thermostability of sarcoplasmic reticulum $\mathrm{Ca}^{2+}$-ATPase.

\section{Calcium uptake capacity of sarcoplasmic reticulum from cockroach leg muscles H. Volmer \\ University of Münster, Zoological Institute, Hindenburgplatz 55, D-4400 Münster, F.R.G.}

The time course of calcium uptake by cockroach (Blaberus fuscus) leg muscle sarcoplasmic reticulum was studied. If calcium uptake was measured in the presence of the calcium-precipitating anion oxalate the following results were obtained: after the initial phase of constant calcium uptake $(0-40 \mathrm{~s})$ the uptake rate rapidly decreased. In the time interval of 2-5 min after the initiation of calcium uptake 
no net calcium uptake took place. Thereafter a decrease of the amount of the accumulated calcium could be observed. The maximum calcium accumulation during the plateau phase was $6.5 \pm 0.5 \mu \mathrm{mol}$ $\mathrm{Ca}^{2+}$ mg protein ${ }^{-1}$.

In the absence of oxalate, a plateau phase could be observed in the time interval of 1-2 min after the initiation of calcium uptake. The calcium accumulation during this phase amounted to 8-12 nmol $\mathrm{Ca}^{2+} \mathrm{mg}^{-1}$. This value is much lower than those found in rabbit skeletal muscle SR [Meissner et al., Biochim. Biophys. Acta 298, 246-69 (1973)]. After the plateau phase again net calcium uptake could be observed. This slow calcium uptake which has earlier been demonstrated in rabbit skeletal muscle SR [Mermier \& Hasselbach, Eur. J. Biochem. 64, 613-20 (1976)] proceeded up to 90-120 min after the initiation of calcium uptake. The calcium accumulation at the end of this second uptake period ranged from $4-12 \mu \mathrm{mol} \mathrm{mg}^{-1}$. High values $(10-12 \mu \mathrm{mol}$ $\mathrm{mg}^{-1}$ ) were found when freshly prepared sarcoplasmic reticulum was used. After the addition of the $\mathrm{Ca}^{2+}$-ionophore A 23187 more than $99 \%$ of the calcium accumulated by the sarcoplasmic reticulum was released indicating that calcium was largely present within the sarcoplasmic reticulum. in the form of free calcium ions.

The results show that even in the absence of oxalate large amounts of calcium can be accumulated by the sarcoplasmic reticulum. Accumulation of free calcium can even be higher than in the presence of oxalate, i.e. at conditions at which calcium. is largely present in the sarcoplasmic reticulum in the form of a calcium oxalate precipitate.

\footnotetext{
Intracellular and superficial cardiac membrane compartments cannot be functionally dissociated by lanthanum: electron probe microanalysis

M. F. Wendt-Gallitelli

Physiologisches Institut II, Universität Tübingen,

Gmelinstrasse 5, D-7400 Tübingen 1, F.R.G.

The hypothesis that contractile responses in cardiac muscle are regulated by superficially located
}

and rapidly exchangeable $\mathrm{Ca}$ was based originally on studies on $\mathrm{Ca}$ fluxes undertaken in the presence and in the absence of $\mathrm{La}$, and on the morphological observation that in heart muscles and cells treated with extracellular La, La normally does not penetrate the plasmalemma [Langer et al., ]. Cell Biol. 54, 441 (1972)]. X-ray microanalysis of guinea-pig papillary muscles (100-300 $\mu \mathrm{M}$ diameter), shockfrozen in a defined functional state, were performed in order to answer two questions. (I) Is it possible with these methods to demonstrate clearly a significant Ca-displacement from superficially located sites after La application? (2) Has La specific effects on cellular structures other than the plasmalemmaglycocalix complex, i.e. are La-characteristic changes in intracellular $\mathrm{Ca}$ distribution detectable with microanalysis?

Force developed by the strips declined completely a few seconds after adding $100 \mu \mathrm{M} \mathrm{LaCl}_{3}$ to the bath solution. After 5-60 min (depending on $\mathrm{Ca}$ and $\mathrm{La}$ concentration and on the rate of stimulation), systolic and diastolic tension increased, exceeding control values. One group of muscles was frozen immediately after complete force decline following La application. The intracellular $\mathrm{Ca}$ distribution was not significantly different from that in untreated muscles. Ca was also well detectable over the cell membrane. A second group of muscles was shock-frozen presystolically at a later time after La application, when systolic and diastolic tension exceeded control values and relaxation was extremely prolonged. At this time, Ca significantly increased in cytoplasm; it disappeared in SR compartments within the vicinity of the cell membrane and at the level of the Z-lines. At this time, small La peaks were clearly detectable intracellulary. Freeze substitution of the remaining portion of the strips used for analysis showed that La was located at the cytoplasmic side of $t$-tubule membranes adjacent to JSR. In the lumen of SR, La precipitates were not detectable. The high sarcoplasmic concentration of $\mathrm{Ca}$ and its disappearance from SR compartments following extracellular $\mathrm{La}$ application, as well as the presence of intracellular La deposits on SR supply evidence that La penetrates the plasmalemma of small normal beating heart muscle preparations and gradually displaces $\mathrm{Ca}$ from the sarcoplasmic reticulum. Therefore, $\mathrm{La}$ cannot be considered a useful tool for dissociating intracellular from extracellular Ca compartments in heart muscle. 


\section{DYNAMICS OF CONTRACTION}

\section{A laser diffractometer for fast sarcomere length measurements in frog single muscle fibres \\ M. A. Bagni, G. Cecchi, F. Colomo \\ Istituto di Fisiologia, Università degli Studi di \\ Firenze, Viale G.B. Morgagni 63, I-50134 Firenze, \\ Italy}

A common method for measuring sarcomere length (SL) is based on the optical properties of muscle fibres. Because of the high regularity in the array of dark and light bands, fibres act as a grating and SL can be calculated by measuring the angle of the first-order diffraction of an incident light beam. The apparatus described here is comparable to diffractometers built previously by other investigators [e.g. Zite-Ferenczi \& Rüdel, Pflüger Arch. 374,97 (1978)], but it has the important advantage of a higher speed of response and of a lower noise; its rise time was less than $5 \mu \mathrm{s}$ and the noise smaller than $0.2 \mathrm{~nm}$ peak to peak. The system is composed by a $5 \mathrm{~mW}$ HeNe laser (Spectra-Physics, model 105), an experimental chamber mounted on the movable stage of a microscope (Carl Zeiss Jena, model Mikro 532) and a position sensor (Quantrad Corporation, model PS-100-10) with associate electronics for computing SL.

The muscle fibre was mounted horizontally and was illuminated by a laser beam of $1 \mathrm{~mm}$ in diameter directed aginst the fibre at an angle of $8^{\circ}$. The position sensor was placed about $150 \mathrm{~mm}$ above the fibre in a plane perpendicular to the plane of the first-order diffracted light. The diffraction angle was calculated with simple trigonometric rules for a right-angle triangle the vertices of which were the centre of the position sensor, the illuminated spot on the fibre and the intersection of the position sensor plane with that perpendicular to the longitudinal axis of the fibre. Sarcomere length was computed according to the general grating equation; calculations were performed in real time by a fast analogue divider (BB, model MPY 100).

The system was designed to work from about $2.1 \mu \mathrm{m}$ to about $2.4 \mu \mathrm{m}$ SL. Within this range of SL the error introduced by change in the angle of the first-order diffraction light place with position sensor plane was negligible. The diffractometer was used for measuring change in SL occurring in fixed end isometric contractions and in responses to length steps imposed at one end of isolated fibres. In addition, the output signal could be used as a servo signal for the length transducer to obtain length clamp conditions of recording.

\section{Transient kinetics of the binding of ATP to covalently crosslinked acto-subfragment-1} T. E. Barman, J. A. Biosca, R. Kassab and F. Travers INSERM U 128 and Centre de Recherche de Biochimie Macromoléculaire, CNRS, B.P. 5051, 34033 Montpellier Cedex, France

The initial steps by which ATP binds to and is hydrolysed by crosslinked acto-subfragment (acto-S1)-I were studied by the rapid flow-quench technique. The experiments were carried out at $15^{\circ} \mathrm{C}$ in $5 \mathrm{mM} \mathrm{KCl}$, pH 8 , with or without $40 \%$ ethylene glycol in the $4-300 \mathrm{~ms}$ time range. Two approaches were used. In the first, crosslinked acto-S1 plus $\left[\gamma-{ }^{32} \mathrm{P}\right] \mathrm{ATP}$ reaction mixtures were quenched in unlabelled ATP, incubated for several turnovers and the ${ }^{32} \mathrm{P}$ determined after a second quench in acid (ATP chase technique). As with S1 and reversible acto-S1 this technique could be used to titrate the active sites of crosslinked acto-SI and also to study the kinetics of the initial binding of ATP. In the second, reaction mixtures were quenched directly in acid and the ${ }^{32} \mathrm{P}_{\mathrm{i}}$ determined ( $\mathrm{P}_{\mathrm{i}}$ burst). Here information on the chemical step can be obtained. In $40 \%$ ethylene glycol, the kinetics of the binding of ATP to crosslinked acto-SI varied linearly with ATP up to $30 \mu \mathrm{M}\left(k\right.$ about $\left.100 \mathrm{~s}^{-1}\right)$ above which the process became too rapid to be followed. The slope of the ATP dependency line is $3.5 \times 10^{6} \mathrm{M}^{-1} \mathrm{~s}^{-1}$. Thus, although a hyperbolic relationship was not obtained (it was with S1 and reversible acto-S1) this low slope suggests a two-step binding process for ATP to the crosslinked complex. In water, the initial binding process was very rapid; at $10 \mu \mathrm{M}$ ATP $k$ is about $80 \mathrm{~s}^{-1}$. Unlike SI and reversible acto-S1 no $\mathrm{P}_{\mathrm{i}}$ burst was obtained with crosslinked acto-S1 in either $40 \%$ ethylene glycol or water.

\section{Undamped elasticity and relaxation in the microsecond range in the activated and relaxed skinned skeletal muscle fibre of the frog \\ T. Blangé, G. J. M. Stienen and B. W. Treijtel Department of Physiology, University of Amsterdam, $1^{2}$ Const. Huygensstraat 20, 1054 BW Amsterdam, The Netherlands}

The tension response of isometrically contracting skinned fibres of the ileofibularis muscle of the frog 
to changes in length completed in $40 \mu \mathrm{s}$ was investigated with respect to the part during and shortly after the length change. The fibres were obtained by the freeze-drying procedure described by Stienen et al. [Pflügers Arch. 397, 272-6 (1983)]. Fibre segments were mounted between a displacement generator and a force transducer with a resonant frequency of about $50 \mathrm{kHz}$. The fibres were incubated and, between measurements, kept in a relaxing solution containing $40 \mathrm{mM} \mathrm{KCl}$, $60 \mathrm{~mm}$ imidazole ( $\mathrm{pH}$ 7), $20 \mathrm{mM}$ EGTA, $5 \mathrm{~mm}$ $\mathrm{MgATP}, \mathrm{pMg}=3,10 \mathrm{mM} \mathrm{PCr}$ and $50 \mathrm{U} \mathrm{ml}^{-1}$ $\mathrm{CPK}$. The tension response in a fully activating Ca-buffered solution consists of a delayed increase (stretch) or decrease (shortening) in tension up to the maximum $T_{1}$, followed by relaxation towards the original isometric level. The tension recordings are simulated by means of a model, in which the influence of density and viscosity of the bathing solution was taken into account. The length of the fibres at $2.15 \mu \mathrm{m}$ sarcomere length varied between 3 and $10 \mathrm{~mm}$. At this sarcomere length the results indicate that the elastic impedance of the fully activated bifre contains an undamped elasticity with a Young's modulus of about $6 \times 10^{7} \mathrm{~N} \mathrm{~m}^{-2}$ and a relaxation time constant of 5-10 $\mu$ s. The undamped elasticity of the relaxed fibre is 5-10 times less, whereas the relaxation time is about the same. The relation between the undamped compliance expressed in $\mathrm{nm}$ per half sarcomere and the isometrically developed tension suggests that a considerable part of the undamped compliance is located in the actin and/or myosin filaments.

\section{Rise of stiffness and isometric tension during a tetanus in frog muscle fibre segments under length clamp conditions}

G. Cecchi, F. Colomo, V. Lombardi and G. Piazzesi Istituto di Fisiologia, Università di Firenze, Viale G.B. Morgagni 63, I-50134 Firenze, Italy

The experiments described in the present report concern the identification of the factors responsible for the faster rise of stiffness with respect to that of tension in an isometric tetanus [Cecchi et al., Science 217, 70 (1982); Ambrogi Lorenzini et al., J. Musc. Res. Cell Motility 4, 177 (1983)]. A first point to clear up is whether tendon lengthening and sarcomere shortening contribute significantly to determine this effect.

Experiments were made at $2-4^{\circ} \mathrm{C}$ on directly stimulated single fibres dissected from the tibialis anterior muscle of the frog. The fibres were mounted in a Ringer trough between the leverarms of a loudspeaker-length transducer and of a capacitance force transducer. The length transducer could be servo controlled by the output signal from a 'striation follower' [Huxley et al., J. Physiol., Lond. 317, 15P (1980)] so as to 'clamp' the length of a tendon free segment along the muscle fibre. Stiffness was determined at increasing tensions throughout the rise and the plateau of isometric tetani. Step-length changes, complete within $120-140 \mu \mathrm{s}$, were imposed at one tendon end by means of the length transducer and the resulting change in tension was measured at the other end.

It was observed (I) that in the range from about $0.5 T_{0}$ to about $0.8 T_{0}$ the ratio of stiffness over isometric tension is the same in whole muscle fibres under fixed end conditions as in the segments under length-clamp conditions and (2) that in either cases the stiffness values were greater, about $20 \%$ at $0.5 T_{0}$ and about $10 \%$ at $0.8 T_{0}$, than those expected for a constant ratio of stiffness over tension. In other words during tetanus rise the development of stiffness led by $10-15 \mathrm{~ms}$ that of tension, irrespective of the length changes in the tendon attachments and in the end-parts of the muscle fibre beyond the edges of the selected fibre segments. Presumably, this phenomenon represents a specific event in the contractile mechanism. As already emphasized by Cecchi $e t$ al. [Science, N.Y. 217, 70 (1982)] it could be explained in terms of a crossbridge model of contraction in which a long-lived state is assumed to exist between attachment of the crossbridges and force generation.

\section{A velocity-dependent shortening deactivation in frog muscle fibres during rise of tension in an isometric tetanus}

F. Colomo, V. Lombardi and G. Piazzesi

Instituto di Fisiologia, Università degli Studi di

Firenze, Viale G.B. Morgagni 63, I-50134 Firenze,

Italy

The finding by Cecchi et al. [J. Physiol. Lond, 285, 257 (1978)], that during the tetanus rise the level of activation (the instantaneous value of $T$, the steady force exerted at any velocity of shortening $V$, and the value of $T_{0}^{*}$, the intercept of the instantaneous $T-V$ curves on the load axis) increases with the time after the beginning of the stimulus volley when test ramp releases are imposed on the fibres, requires further attention. If activation is rising rapidly throughout the early part of a tetanus, why does not also the value of $T$ during constant velocity releases rise continuously?

Experiments were performed at low $\left(2-4^{\circ} \mathrm{C}\right)$ and room $\left(19-22^{\circ} \mathrm{C}\right)$ temperatures on directly 
stimulated single fibres dissected from the tibialis anterior muscle of the frog. Force-velocity data points were obtained by imposing ramp releases at one tendon end of the fibres, tensions were measured at the other end. Simple and double ramp releases were used. In each instantaneous $T-V$ relation determined during the early part of a tetanus, the various values of $T$ obtained in response to test releases at different velocities were potentiated or depressed by previous shortening. Conditioning shortenings at lower speeds determined potentiation and, at higher speeds, depression. Both these effects, in the range of releases smaller than $0.05 l_{0}$ (the fibre length at about $2.25 \mu \mathrm{m} \mathrm{SL}$ ) at least, were independent of the amount of shortening. At any present time during the early part of a tetanus an inverse relation was found between the potentiated or depressed values of $T$ and the actual velocity of conditioning shortening. Finally, if a series of successive ramp releases at the same velocity was imposed on the fibres at various increasing times during the tetanus rise, the time required by $T$ to attain $90 \%$ of its steady-state value was shorter the smaller the shortening velocity used. In general, an inverse correlation is shown to exist during the early part of a tetanus between the velocity of shortening and the rate of development of activation.

\section{Economy of force maintenance in rat myocardium as modulated by thyroid gland activity and in vitro temperature changes C. Holubarsch ${ }^{1}$, R. Z. Litten ${ }^{2}$, L. A. Mulieri ${ }^{2}$ and N. R. Alpert ${ }^{2}$ \\ 'University of Freiburg, Cardiology, F.R.G. \\ ${ }^{2}$ University of Vermont, Burlington, Department \\ of Physiology and Biophysics, Vermont, U.S.A.}

Using planar antimony-bismuth thermopiles we measured total activity-related heat of left ventricular papillary muscles liberated during experimentally induced tetanic contractions under conditions of 1 I mM calcium, $7.5 \mathrm{~mm}$ caffeine, and a high stimulation rate at $21^{\circ} \mathrm{C}$. The slope of the function of total activity-related heat versus developed tension-time integral yields a measure of economy of force development in the preparation. It can be shown that the slope is decreased by $56 \%$ in a hypothyrotic rat group (propylthiouracil treated) compared to the control group. Moreover, this change in economy is associated with a change in the content of heavy chain $\beta$ of myosin ( $10 \%$ in control and $90 \%$ in hypothyrotic rats) as well as a $72 \%$ decrease in myofibrillar myosin $\mathrm{Ca}^{2+}$-stimulated ATPase activity. We tried to mimic these chronic changes of economy of force development by varying acutely the in vitro temperature. In the control myocardium, when the temperature was lowered by $10^{\circ} \mathrm{C}$, almost the same economy was found as in the propylthiouracil-treated rat myocardium at $21^{\circ} \mathrm{C}$ $\left(Q_{10}=2.09\right)$. In the hypothyrotic myocardium, we increased the temperature to $31^{\circ} \mathrm{C}$ and found a $Q_{10}$ of 3.09. The data show that a change of the isoenzyme composition in the myocardium can be mimicked by adequate changes of the in vitro temperature. Furthermore, a synopsis of the biochemical and biophysical data obtained permits a separation between cycling rate changes of crossbridges on the one hand, and alterations of force generating on-time of crossbridges on the other.

\section{Myosin isozymes and force-velocity relation in fast rat muscle}

A. Jaafar, Godelieve Beckers-Bleukx and

G. Maréchal

Department of Physiology, University of Louvain,

B-1200 Brussels, Belgium

The force-velocity relations of 43 extensors digitorum longus (EDL) muscles of 90-day-old male $(n=24)$ and female $(n=19)$ rats were measured in vivo after anaesthetizing the animals with Thalamonal. Direct stimulation of $100 / \mathrm{s}$ for $0.3 \mathrm{~s}$ duration was used; the temperature was kept constant at $37^{\circ} \mathrm{C}$ by dripping warm Krebs around the perfused muscles. Shortening was effected at eight different velocities under the control of an ergometer and the tension was recorded on a fast u.v. recorder. The velocity constants (B) were computed using a form of Aubert's equation $P=P_{0} \exp ^{(-V / B)}$ (Aubert, 1956): they varied between 0.9 and 2.55 muscle length/s.

The myosin isozymes of the muscles were separated by electrophoresis of native myosins in a nondissociating medium into three zones: $\mathrm{HC}-\mathrm{LC}_{1} \mathrm{f}-\mathrm{LC}_{1} \mathrm{f}\left(\mathrm{FM}_{3}\right) ; \mathrm{HC}-\mathrm{LC}_{1} \mathrm{f}-\mathrm{LC}_{3} \mathrm{f}\left(\mathrm{FM}_{2}\right)$; and $\mathrm{HC}-\mathrm{LC}_{3} \mathrm{f}-\mathrm{LC}_{3} \mathrm{f}\left(\mathrm{FM}_{\mathrm{T}}\right)$; i.e. only the fast type isomyosins were found in EDL muscles. The relative amount of $\mathrm{FM}_{1}, \mathrm{FM}_{2}$ and $\mathrm{FM}_{3}$ was measured by computer planimetry and the concentration of $L C_{1} f$ relative to the total concentration of the alkaline light chains $\left(\mathrm{LC}_{1} \mathrm{f}+\mathrm{LC}_{3} \mathrm{f}\right)$ was computed as $\left(\mathrm{FM}_{3}+1 / 2 \quad \mathrm{FM}_{2}\right) /\left(\mathrm{FM}_{1}+\mathrm{FM}_{2}+\mathrm{FM}_{3}\right)$ : it varied between 0.50 and 0.68 .

Although the individual variations of the velocity constant and the proportions of $\mathrm{LC}_{\mathrm{I}} \mathrm{f}$ were large, we observed no correlation between them. Also there were no differences between muscle of male and female animals in respect to velocity 
constant (B) or the amount of myosin isozymes These experiments provide no support for the hypothesis that the variations in power or maximum velocity of fast muscle is controlled by the relative proportion of the two alkaline light-chains, $\mathrm{LC}_{\mathrm{I}} \mathrm{f}$ and $\mathrm{LC}_{3} \mathrm{f}$.

\section{A simple explanation of 'force enhancement' during stretch \\ R. Jarosch \\ Institut für Botanik der Universität, \\ Lasserstrasse 39, A-5020 Salzburg, Austria}

It is known in whole muscle and in single fibres that an increase in sarcomere length during contraction results in an increase in tension [Edman et al., In Cross-bridge Mechanics in Muscle Contraction (edited by Sugi \& Pollack), pp. 297-310, University Park Press, (1979)]. This extra tension is 'without increase in the rate of ATP splitting' [Woledge \& Curtin, In Cross-bridge Mechanics in Muscle Contraction (edited by Sugi \& Pollack), pp. 473-80, University Park Press (1979)]. The observed tension transients are specific for each kind of muscle, and the current sliding filament model is unable to account for the phenomenon of stretch activation [Steiger, In Cross-bridge Mechanics in Muscle Contraction (edited by Sugi \& Pollack), pp. 259-74, University Park Press (1979)]. Its mechanism remains one of the main unsolved problems in muscle research [Squire, The Structural Basis of Muscle Contraction, New York, Plenum, (1981)].

The explanation of this phenomenon is quite simple when we consider the mechanically effective helical surface of the thin filament. In order to put a screw into a wall I can press it in or better, I can screw it in. The screw is rotated passively in the first case and actively in the second. Since the myosin crossbridges of an activated muscle are locked on the surfaces of the steep thin filament double helices, the sliding motion between thick and thin filaments is absolutely connected with rotations of the thin filaments. Therefore every stretch of an activated muscle produces passive rotations of the thin filaments. Since these are anchored at the Z-discs the extra tension is 'sensed' presumably by the anchoring coiled-coil filaments (tropomyosin, $a$-actinin) whose torsional tension increases additionally to the already working torsional tension that is produced by the coiled-coils during the Ca-activation. This torsional tension is supposed to produce the screwing motion of the thin filament helices during isotonic contraction, or the tonic force, if the muscle is held isometrically
[Jarosch, In Cell Motility: Molecules and Organization (edited by Hatano et al.), pp. 291-319, University of Tokyo Press (1979); Jarosch \& Foissner, Eur. J. Cell Biol. 26, 295-302 (1982)]. The variable torsional tension may also be responsible for the dynamic structure of the Z-discs that vary as a function of sarcomere length (Squire, 1981). The enhanced force may appear delayed because the transformation of the tensile stress in torsional tension by the passive friction-dependent rotations (heat) needs some time. The 'force reduction' after quick release [Podolsky, In Molecular. Basis of Motility (edited by Heilmeyer et al.), pp. 53-68, Springer, Berlin (1976)] can be interpreted as the opposite process of 'force enhancement'. The screw-mechanical origin of the extra force is demonstrated on the poster by a functional spring-model of a sarcomere.

\section{Effect of ATP, ADP and vanadate on the functional properties of actomyosin} A. Köver ${ }^{1}$, S. Csabina ${ }^{1}$, Á. Szöör ${ }^{2}$ and J. Csongor ${ }^{1}$ ${ }^{1}$ Central Research Laboratory and ${ }^{2}$ Department of Physiology, University Medical School, Debrecen, Hungary

The modifying effect of vanadate ions on the interaction between actomyosin and nucleotide has been studied. It has been found that the length of clearing period induced by ATP increased depending on the concentration of vanadate ions. A fast superprecipitation occurred with the mixture of 0.I $\mathrm{mM}$ ATP and $1.0 \mathrm{mM}$ ADP without vanadate, but the presence of any vanadate ions resulted in a prolonged clearing. ADP (free of ATP) had no effect on the turbidity of the suspension either in the presence or in the absence of vanadate ions. When the solution contained myokinase but no vanadate, the ADP (free of ATP) changed the turbidity of the actomyosin suspension in the order of superprecipitation-clearing - superprecipitation. In the solution containing vanadate, a fast superprecipitation followed by a prolonged clearing occurred. The pyrophosphate which itself has no effect on the turbidity of the protein suspension induced a prolonged clearing in the presence of vanadate. The synthesis of ATP, which took place from the ADP produced during superprecipitation [Csabina et al., Acta Biochim. Biophys. Acad. Sci. Hung. 18, 231 (1983)], could be partially inhibited by diadenosine-pentaphosphate, a specific myokinase inhibitor both in the presence and absence of vanadate ions. 
Mechanochemistry of ATP binding to myosin heads in skinned Limulus fibres and skinned heart muscle fibres

H. J. Kuhn, R. Kulik, H. Winker and S.Pferrer Abteilung für Allgemeine Physiologie, Universität Ulm, D-7900 Ulm. G.F.R.

Glycerinated telson levator fibre bundles of Limuius and fibre bundles from the right ventricle of porcine hearts (subendo cardial layers) contract when incubated in sait solutions containing $9.5 \mu \mathrm{M}$ $\mathrm{Ca}^{2+}$ and 15.0-0.025 mM ATP (pH 6.7-7.0). ATPase activity increases with increasing the $\mathrm{MgATP}$ concentration of the bathing solution. Maximal rates of ATP hydrolysis were $1 \mathrm{~s}^{-1}$ and $0.3 \mathrm{~s}^{-1}$ per myosin head in Limulus and heart muscle preparation, respectively. Half maximal rates of ATP hydrolysis were observed at $130 \mu \mathrm{M}$ and $260 \mu \mathrm{M} \mathrm{MgATP}$ for Limulus and heart muscle preparation, respectively.

Tension changes following quick release steps show the four phases characteristic of striated muscle: (1) the elastic phase; (2) a quick relaxation phase with components A-C: (3) a delayed phase of tension adjustment; and (4) a slow phase of relaxation. In both muscle types, phases 2-4 become gradually faster when the MgATP concentration of the bathing solution increases. Maximal rate constants of phase 4 correspond to the maximal rate of ATP hydrolysis characteristic of the muscle type. In Limulus fibres the rate constants of phase 2 and 3 follow the MichaelisMenten law; Michaelis-Menten constants depend markedly on the amount and the direction of the applied length change: $K_{\mathrm{m}}$ values of phase 3 were $0.8,1.3,1.1$ and $0.6 \mathrm{~mm}$ (and of phase 2: 0.6., 1.6, 1.1 and $0.6 \mathrm{~mm}$ ) at $-12,-6,6$, $12 \mathrm{~nm} / \mathrm{HS}$ length change. The dependence of the Michaelis-Menten constants on fibre lengthening indicates that the apparent dissociation constant from the enzymatic sites of the myosin heads transiently decreases when the fibre is stretched and released. We interprete these results as a $\mathrm{MgATP}$-induced detachment of crossbridges from the actin during phase 2 .

\footnotetext{
The mechanochemical capability of cleaved heavy myosin subfragment-1

R. Levy and A. Oplatka

The Weizmann Institute of Science,

Rehovot 76 100, Israel

Mechanochemical energy transformation in actomyosin systems is usually believed to be the outcome of conformational changes in the myosin heads (heavy meromyosin subfragment-1, or SI,
}

when isolated). Upon illustrating such a change, the heavy chain of the protein is drawn in different conformations. Moreover, the mechanical force is supposed to be generated and transmitted along this continuous backbone. Breakdown of S1 into segments could, therefore, lead to uncoupling of mechanochemical transduction. It has recently been reported [Chaussepied et al, FEBS Lett. 161, 84 (1983)] that SI can be split by staphylococcal protease into three segments. The modified protein had intact actin-activated ATPase. We checked its capability to cause (under the microscope) contraction of myofibrils from which myosin had been extracted ('ghosts'). Contraction took place upon adding MgATP (as with the original SI), suggesting that if indeed force is associated with conformational changes then hydrogen bonds, salt linkages and hydrophobic bonds, which hold together the three segments, must be utilized for the generation and transfer of force, rather than the polypeptide backbone. Trypsin-digested SI did not cause contraction and this is probably due to its low affinity to actin, which is responsible for lower $\mathrm{Mg}$ activated ATPase activity at nonsaturating actin concentrations (Chaussepied et al., 1983). An alternative interpretation of our findings would be that it is the continuous aqueous medium which serves as the transmitter of mechanical momenta rather than a continuous protein network.

\section{Developed force depends on diastolic length in mammalian ventricular muscle}

C. G. Nichols and B. R. Jewell

Department of Physiology. The University of

Leeds, Leeds LS2 9NQ, U.K.

We have recently shown for the first time that the length of the muscle between contractions is important in determining contractility [Jewell \& Nichols, J. Physiol, Lond. 346, 68P (1984)]. After diastolic release, when the muscle rests at a short length but continues to contract at a control length, developed force declines slowly over about $10 \mathrm{~min}$ (at $30^{\circ} \mathrm{C}$ ) to a new steady level. If the muscle is then held once more at the control length, developed force slowly returns to control. This result would suggest that during each diastolic interval a change in the 'inotropic state' of the muscle is occurring. The change between each beat is actually very small and only becomes appreciable over a period of minutes. This raises the possibility that the accumulation of the effect may require excitation or contraction. To test this 
possibility, the following experiment was performed on isometrically contracting cat papillary muscles.

(1) Diastolic length was reduced from control for $10 \mathrm{~min}$ during which time developed force fell to a new level. Diastolic length was then returned to control and the developed force slowly recovered. (2) The muscle went through the same length changes as in (1) for $10 \mathrm{~min}$ but was not stimulated. Stimulation was then restarted and developed force recovered in a 'staircase' manner back to control. (3) The muscle was not stimulated for $10 \mathrm{~min}$ during which time muscle length was unaltered. On restarting stimulation developed force again recovered in a 'staircase' manner.

The recovery in (3) differed from the recovery in (2). By expressing developed force in each contraction of the recovery in (2) as a fraction of the developed force in the corresponding contraction in the recovery in (3) it was shown that the difference is attributable to an additional recovery in (2) which is very similar to the recovery in (1). This shows that the inotropic changes resulting from altered diastolic length can occur whether or not the muscle contracts during the period of accumulation. [Supported by the British Heart Foundation (grant 81/58). C.G.N. is a holder of a SERC(CASE) award.]

\section{Length dependence of the short-term force-interval relationship in rat myocardium C. Poggesi, R. Bottinelli and R. Minelli Istituto di Fisiologia umana, Università di Pavia, Pavia, Italia}

In mammalian heart muscle the analysis of the inotropic effects due to the alteration of a single interval of excitation may give some information about the cellular calcium fluxes that activate and regulate contraction. Since contractile activation of cardiac muscle is supported to be influenced by muscle length, in the present study we examined. whether, in isolated rat myocardium, mechanical restitution, postextrasystolic potentiation and its decay are dependent on the resting muscle length.

Papillary muscles $\left(26^{\circ} \mathrm{C}\right)$ were stimulated to contract isometrically at constant control interval. In these steady-state conditions a single test stimulus was applied to the muscle at a shorter interval (test interval) after a control stimulus. The resulting test contraction was depressed, as expected from the phenomenon of mechanical restitution. After the test stimulus, the control interval of stimulation was resumed. The contraction next to the test one was potentiated (postextrasystolic potentiation). Complete removal of the potentiated state induced by the test contraction took some beats to occur (potentiation decay). When the preparation had again reached the steady-state conditions, the effects of a new test interval could be analysed. Test intervals longer than the control interval were also tested. In order to study the influence of muscle length on these phenomena, the experimental procedure described was repeated at $L_{\max }$ (the length at which developed force was maximal) and at a shorter length $\left(0.9 L_{\text {max }}\right)$.

Results obtained showed that all the phenomena under study were influenced by the resting muscle length. (I) At test intervals shorter than the control one, the strength of test contractions increased with the interval towards the control values; restitution curves showed that this recovery occurred faster at $L_{\max }$ than at the shorter length. At test intervals longer than the control one, the strength of test contractions became greater than that of the control beat and the effect of length was reversed, i.e. a smaller increase occurred at $L_{\max }$. (2) At any test interval determining potentiation, the potentiating effect was smaller at $L_{\max }$ than at the shorter length. (3) In all conditions the decay of potentiation was an exponential function of the number of beats following the test contraction. Evaluation of the exponential beat constants of the decay showed that the potentiation was lost with a faster rate at the shorter length than at $L_{\max }$. These results suggest that muscle length affects more than one step of the $\mathrm{E}-\mathrm{C}$ coupling processes of rat myocardium.

\section{Simple method for comparison of myofibrillar ATPase activity in stretched and regenerating skeletal muscle fibres}

J. Rohán and F. Guba

Institute of Biochemistry, University of Medical

Sciences, Szeged, H-6701, P.O.B. 415, Hungary

Fixation of rat muscles in stretched position results in fibre 'splitting' after one week. This phenomenon was investigated at both the light and electron microscopic levels. The time course of the process strongly supports the idea that the virtual fibre splitting is a real myotube fusion which follows the phagocytosis of some muscle fibres. The surviving basement membrane remains visible for a couple of weeks after the fibre necrosis and following phagocytosis. These basement membranes often bears inclusions. We compared the ATPase staining intensity of 'split' fibres with fibres regenerating after a necrosis induced by 
rapid freezing of the muscle. Since the comparison of the staining intensity of two independent sections is uncertain, the different preincubations were carried out in the same section. This way we found that the staining of the rat soleus SO fibres is independent of preincubation. The fast oxidative fibres (FOG) lost their staining after preincubation at $\mathrm{pH}$ 4.6 or 4.35 to different degree. In the medial and intermediate parts of rat gastrocnemius muscle the behaviour of fast fibres is similar to the soleus FOG fibres, while staining intensity of the slow fibres becomes much stronger after the preincubation. In a part of the gastrocnemius intermedius the staining of the slow fibres is unchanged after preincubation at $\mathrm{pH} 4.35$, similar to the soleus SO fibres. We examined the staining properties of the IIC fibres in regenerating rat soleus after injury caused by rapid freezing. Seven days after the necrosis the regenerating fibres showed a dark and homogeneous staining at $\mathrm{pH} 9.2$ corresponding to the normal fast fibres. The regenerating area preincubated at $\mathrm{pH} 4.6$ shows a mosaic pattern and the staining intensity of the IIc fibres was found between the normal soleus slow and the light, fast fibres. The staining properties of the 'split' fibres in stretched soleus of the rat correspond to those of the regenerating fibres. The stretched rat soleus on the basis of ATPase staining becomes slower. The rabbit soleus fixed in a shortened position loses its homogeneity and shows a mosaic pattern at $\mathrm{pH}$ 4.35 , and so becomes faster.

\section{Segmental differences in maximum speed of shortening and myofibrillar ATPase activity in frog skeletal muscle fibres}

G. te Kronnie, C. Reggiani and K. A. P. Edman Department of Pharmacology, University: of Lund, S-223 62 Lund, Sweden

Markers of black dog's hair were placed, $0.6-0.8 \mathrm{~mm}$ apart, on the upper surface of single fibres of the anterior tibialis muscle of $R$. temporaria, and the distance between two adjacent markers (one segment) was measured by a photoelectric detector system [Edman \& Reggiani,
J. Physiol, Lond. 351, 169-98 (1984)]. The fibre was stimulated supramaximally (massive field stimulation) at $2 \mathrm{~min}$ intervals to produce a $0.5 \mathrm{~s}$ fused tetanus at $2.25 \mu \mathrm{m}$ sarcomere length $\left(1-2^{\circ} \mathrm{C}\right)$. The maximum velocity of shortening, $V_{0}$, was recorded by releasing the fibre to shorten against a small load (about $2 \%$ of the tetanic force). $V_{0}$ varied substantially in different segments along the fibre, each fibre exhibiting a unique $V_{0}$ pattern which was maintained during the whole experiment [also see Edman \& Reggiani, J. Physiol, Lond. 329, 47-8P (1982)]. In 25 preparations the difference between the highest and lowest $V_{0}$ values in a given fibre ranged between 10 and $55 \%$ of the fibre mean.

In 12 fibres, segment $V_{0}$ measurements were correlated with the calcium-activated myofibrillar ATPase activity using a quantitative histochemical technique [Van der Laarse et al., Histochem. J. 16, 529-41 (1984)]. From selected fibre segments (exhibiting marked differences in $V_{0}$ ) transverse serial cryosections (thickness $16 \mu \mathrm{m}$ ) were collected on microscope slides. The sections were incubated for $15 \mathrm{~min}$ at $18^{\circ} \mathrm{C}$ in a buffered medium (pH 7.20) containing $2.5 \mathrm{~mm} \mathrm{ATP}$ and $0.09 \mathrm{~mm}$ $\mathrm{CaCl}_{2}$. The absorbance of lead sulphide precipitate (proportional to liberated inorganic phosphate) was measured in a representative area (diameter $15 \mu \mathrm{m}$ ) of each section using a Zeiss cytospectrophotometer (wavelength $500 \mathrm{~nm}$ ). The cumulative absorbance of all sections from one segment was normalized with respect to the length of the segment. This value, $A_{\text {norm }}$, was used as a measure of the myofibrillar ATPase activity in that segment. Differences in $A_{\text {norm }}$ of up to $38 \%$ were recorded between individual segments of a fibre. The regression of $A_{\text {norm }}$ upon $V_{0}$ was calculated for 26 segments of 12 fibres using the least squares method. This analysis showed a statistically significant correlation between $A_{\text {norm }}$ and $V_{0}(P<0.01)$. These results therefore suggest that the segmental differences in $V_{0}$ existing along a muscle fibre may, at least partly, be explained by local differences in myofibrillar ATPase activity.

\section{EXCITATION-CONTRACTION COUPLING}

How does increasing $\left[\mathrm{Ca}^{2+}\right]_{0}$ decrease force in Ca-overloaded cardiac muscle?

D. A. Eisner, D. G. Allen, J. S. Pirolo and

G. L. Smith

Department of Physiology, University College

London, Gower Street, London WC1E 6BT, U.K.
Under normal conditions increasing the external calcium concentration $\left(\left[\mathrm{Ca}^{2+}\right]_{0}\right)$ increases the force of contraction of cardiac muscle. However when the Na-K pump is inhibited and therefore $\left[\mathrm{Ca}^{2+}\right]_{i}$ is elevated, increasing $\left[\mathrm{Ca}^{2+}\right]_{0}$ decreases the force. This negative inotropic effect of $\left[\mathrm{Ca}^{2+}\right]_{0}$ has been 
termed 'Ca-overload' [Vassalle \& Lin, Am. J. Physiol. 236, H689-97 (1979)].

The mechanism is, however, unknown. We have measured $\left[\mathrm{Ca}^{2+}\right]_{i}$ using the photoprotein aequorin in ferret papillary muscles. Increasing $\left[\mathrm{Ca}^{2+}\right]_{0}$ (from 2 to $10 \mathrm{~mm}$ ) in the presence of strophanthidin $(10 \mu \mathrm{M})$ decreased the force. The peak aequorin signal increased suggesting that the decrease of force is not due to a decrease of $\left[\mathrm{Ca}^{2+}\right]_{i}$ : and may instead be due to a decrease of Ca-sensitivity of the contractile proteins [cf. Wier \& Hess, J. gen. Physiol. 83, 395-415 (1984)]. However, the following observations suggest that the fall of force in $\mathrm{Ca}$ overload is not produced by a simple decrease of Ca sensitivity.

(1) In $10 \mathrm{~mm}\left[\mathrm{Ca}^{2+}\right]_{0}$ the peak aequorin signals are very variable in magnitude. (2) Diastolic $\mathrm{Ca}$ oscillations [Orchard et al, Nature, Lond. 304, 735-8 (1983)] are seen following stimulation. These are due to release of $\mathrm{Ca}$ from the sarcoplasmic reticulum. The latency of these oscillations after the stimulus varies from beat to beat. (3) There is an inverse correlation between the magnitude of the peak aequorin signal on stimulation and that of the preceding diastolic oscillation: larger oscillations are followed by smaller peak aequorin signals. It is likely that the increased variability of the peak aequorin signal reflects the fact that different cells have different systolic $\left[\mathrm{Ca}^{2+}\right]_{\mathrm{j}}$. We suggest that this nonuniformity of activation may contribute to the fall of force seen in Ca-overload. The variability of the peak aequorin signals may originate because the oscillatory release of calcium from the sarcoplasmic reticulum decreases the amount of Ca released by a subsequent stimulus. (Supported by M.R.C. and British Heart Foundation. J.S.P. is a Sarnoff Fellow.)

\footnotetext{
Quin 2 as an indicator for $\mathrm{Ca}^{2+}$ concentrations under resting and activated conditions in barnacle single muscle fibres

J. Godber ${ }^{1}$, M. P. Timmerman ${ }^{1}$, A. Walton ${ }^{2}$ and

C. C. Ashley ${ }^{1}$

University Laboratory of Physiology,

Parks Road, Oxford OX1 3PT, U.K.

${ }^{2}$ Open University, Boars Hill, Oxford, U.K.

Single isolated Balanus nubilus muscle fibres were cannulated and axially injected with the fluorescent $\mathrm{Ca}^{2+}$ indicator quin 2 [Tsien et al., J. Cell Biol. 94, 325 (1982)] and after $30 \mathrm{~min}$ for radial diffusion of the quin 2 , were perfused with cold saline $\left(15^{\circ} \mathrm{C}\right)$
}

in a chamber. The quin 2 was excited at $339 \mathrm{~nm}$ using a $150 \mathrm{~W}$ Xenon lamp and a $12001 / \mathrm{mm}$ grating monochromator. Calcium-dependent fluorescence was recorded with either microscope optics, $\times 40$ objective and a $510 \mathrm{~nm}$ glass filter (Corning 3.73 ) or with light guide optics onto a photomultiplier tube at $90^{\circ}$ to the incident beam. Noncalcium-dependent fluorescence was monitored as a control at $600 \mathrm{~nm}$. Resting free $\mathrm{Ca}^{2+}$ levels were related to fluorescence by the equation $\left[\mathrm{Ca}^{2+}\right]=K_{\mathrm{d}}\left(F-F_{\min }\right) /\left(F_{\max }-F\right)$. Maximum fluoresence $\left(F_{\max }\right)$ was obtained either by fibre damage or by applying $20 \mathrm{~mm}$ caffeine solution. Minimum fluorescence $\left(F_{\text {min }}\right)$ was found by washing the fibre repeatedly in $\mathrm{OCa}(\mathrm{Mg}$ substituted) saline or $20 \mathrm{~mm} K_{2} E G T A$. $K_{d}$ at fibre $\mathrm{Mg}$ concentrations ( $5 \mathrm{~mm}, \mathrm{pH} 7.1$ ) is $457 \mathrm{~mm}$ (Harvey et al., 1985, in press). The mean resting free $\mathrm{Ca}^{2+}$ concentration was $297 \mathrm{nM} \pm 21 \quad(N=3)$. Fluorescence changes were also recorded under voltage clamp conditions. Intracellular quin 2 concentrations ranged from 8-450 $\mu \mathrm{M}$. The fast reaction kinetics and $1: 1$ stoichiometry of quin 2 for $\mathrm{Ca}^{2+}$ [Tsien, Biochemistry 19, 2396 (1980)] make it an accurate direct indicator for the free $\mathrm{Ca}^{2+}$ transient. Upon stimulation, fluorescence rises before force, attaining a maximum value when the rate of rise of force is greatest. Mechanical relaxation has a shorter half time than fluorescence decrease. Significant buffering of $\mathrm{Ca}^{2+}$ released upon activation was observed at high quin 2 concentrations, as shown by an inverse relation between quin 2 concentration and force, and the slower decay of the fluorescence transient at higher quin 2 concentrations. $\left[t_{1 / 2}<800 \mathrm{~ms}\right.$ at $8 \mu \mathrm{M}$ intracellular quin 2 concentration (pulse duration $=400 \mathrm{~ms}$ ) $7 \mathrm{~s}$ at $450 \mu \mathrm{M}$. Due to its relatively high affinity for $\mathrm{Ca}^{2+}$, the quin 2 readily saturates at greater stimulation intensities. Thus as stimulation intensity is increased, force transient buffering is observed, while the fluorescence signal increases in amplitude. Upon quin 2 saturation, no further increase in fluorescence is seen, but now the force transient increases in amplitude. To obtain spatial information of $\mathrm{Ca}^{2+}$ release sites along the muscle, quin 2 has also been used in fibres viewed under a microscope image intensification system [Reynolds, In Detection and Measurement of Free Calcium in Cells (edited by Ashley \& Campbell), Amsterdam, North Holland (1979)]. An increase in fluorescence was recorded using a single voltage clamp pulse. There was evidence of banding produced by subcellular structures [Ashley et al., J. Physiol. Lond. 384, 8P.] (Supported by M.R.C. Partnership Award.) 
Localization of intracellular calcium release in cardiovascular muscle cells

Kent Hermsmeyer and Michael Sturek

Department of Pharmacology and The

Cardiovascular Center, University of Iowa,

Iowa City, Iowa 52242, U.S.A.

Using our new method for optical quantification of intracellular free $\mathrm{Ca}^{2+}$ concentration, or $\mathrm{Ca}^{2+}$ activity, we localized release of $\mathrm{Ca}^{2+}$ in single cultured cardiac and vascular muscle cells. The metallochromic dye, arsenazo III (Az III), was loaded into muscle cells by exposure for 1-15 min to liposomes containing dye solution. With intracellular Az III concentrations of $200-300 \mu \mathrm{M}$, we could quantify $\mathrm{Ca}^{2+}$ release in $20 \mu \mathrm{m}^{2}$ localized areas of a single cell and visualize organelles with interference contrast light microscopy. The movement signals were measured and corrected for by light at 580 and $702 \mathrm{~nm}$, allowing the purely $\mathrm{Ca}^{2+}$ sensitive portion of the $660 \mathrm{~nm}$ signal to be isolated. An imaging diaphragm in the focal plane of the final imaging lens of the Leitz MPV photometer allowed precise positioning for measurements less than $I \mu \mathrm{m}$ apart. Both cardiac and vascular muscle cells showed great spatial inhomogeneity of $\mathrm{Ca}^{2+}$ activity during spontaneous contraction. Peak $\mathrm{Ca}^{2+}$ activity could easily be five times that in the adjacent area only $I \mu \mathrm{m}$ away. The areas of high amplitude $\mathrm{Ca}^{2+}$ activity also exceeded the average $\mathrm{Ca}^{2}+$ activity for the whole cell by more than five times, suggesting that $\mathrm{Ca}^{2+}$ activity is locally very high. In $1 \mathrm{nM}$ norepinephrine, amplitude and duration of the $\mathrm{Ca}^{2+}-\mathrm{Az}$ III signal increased in both cardiac and vascular muscle cells. The greatest increases in $\mathrm{Ca}^{2+}$ activity occurred in areas which showed only little or moderate $\mathrm{Ca}^{2+}$ release during spontaneous contractions. Because the release of $\mathrm{Ca}^{2+}$ appears to be localized to less than $1 / 3$ of the volume of the cell, $\mathrm{Ca}^{2+}$ that is allowed access to the myofibrils must be strongly influenced by absorption, sequestration, or removal. Therefore, in both cardiac and vascular muscle cells, $\mathrm{Ca}^{2+}$ release and active $\mathrm{Ca}^{2+}$ buffering appear more important than diffusion for intracellular $\mathrm{Ca}^{2+}$ regulation.

\author{
Mechanism of excitation-contraction coupling \\ at the isolated striated fibre level in the \\ experimental muscular dystrophy induced by \\ 2,4-dichlorophenoxiacetic acid \\ M. Isac and Rodica-Maria Isac \\ Biophysics Research Group, Romanian \\ Department of Biology, A1.I.Cuza University, \\ lassy, Romania
}

The effects of 2,4-dichlorophenoxyacetic acid (DCPAA) were studied on isolated muscle fibres of the frog. There is evidence that drugs may affect contractility in skeletal muscle either by exerting an effect on plasma membrane and thereby changing the kinetics of the action potential or by influencing the function of intracellular organelles involved in the metabolism of activator calcium. Our experiments included measurement of isometric twitch responses and membrane action potential and contractures produced by caffeine and increased potassium concentration. DCPAA increased the amplitude of the isometric twitch of frog single muscle fibres at a low concentration. The rate of rise of tension was unchanged. The rate of relaxation was reduced and the action potentials showed a marked decrease in the rate of repolarization leading to a prolongation of the action potential. Experiments carried out in calcium-free medium suggested that twitch potentiation by DCPAA was not due to increased transmembrane calcium influx. The contractile response produced by caffeine and by high potassium concentration was unaffected. The results are consistent with the view that twitch potentiation by DCPAA is due to increased release of calcium from the cellular stores caused by prolongation of action potential. In frog muscle, the DCPAA probably also reduces the rate of sequestration of activator calcium during the relaxation phase. Replacing calcium with lanthanum restores the resting membrane potential and thus the excitability of the muscle fibre and gives a normal twitch response. This finding suggests that skeletal muscle does not immediately depend on extracellular calcium influx for its contractile activity. Calcium is released and taken up again by the tubular system of SR, i.e. the muscle fibre behaves virtually as a closed system in this sense. In some experiments we investigated also the effects of DCPAA on the contractile response and action potential of lumbrical muscle from rats.

\section{Optical and mechanical signals during excitation-contraction coupling in single fibres of frog skeletal muscle}

\author{
H. Oetliker and R. A. Schümperli \\ Physiological Institute, University of Berne, \\ Buehlplatz 5, 3012 Bern, Switzerland
}

Isolated single fibres of frog skeletal muscle undergo a transient decrease in optical retardation 
upon activation. This was named early large birefringence signal (BS) [Baylor \& Oetliker, J. Physiol, Lond. 264, 14I-62 (1977)]. The BS starts $\sim 0.8 \mathrm{~ms}$ after onset of the action potential (measured with a potentiometric dye). The BS was assumed to reflect changes in SR-membrane potential or conformation or conformational changes in the contractile proteins. On energetic grounds, the BS cannot reflect changes in SR-membrane potential [Oetliker, J. Musc. Res. Cell Motility 3, 247-72 (1982)]. Because of the temporal proximity to latency relaxation (LR) one wonders if the BS could be an optical correlate of $L R$. The records published so far from point stimulated fibres where a transducer with a resonance frequency of $\sim 500 \mathrm{~Hz}$ was used showed a ms-delay between BS and LR. But, it is not clear how much of this delay is due to differences in response time of the detection systems. The measurements were therefore repeated with a piezoelectric transducer $(>3300 \mathrm{~Hz})$ and force output was calculated as described in Ford et al. [J. Physiol., Lond. 269, 441-515 (1977)]. The new transducer was shown to measure faithfully onset of tension. Under field stimulation in normotonic Ringer ( $\mathrm{IT}$ ) and $20^{\circ} \mathrm{C}$, the interval between the early phases of the BS and LR (scaled to have the same slopes) is of the order of $360 \mu$ s. Treatments known to delay contractile activation, such as hypertonicity, increase the interval between the two signals. In addition, when tonicity was increased above $2.2 \mathrm{~T}$, most single fibres clearly showed an increase in tension preceding the onset of LR. In the records with the highest signal to noise ratios, it appeared that this tension increase (named prerelaxation-contraction, PRC) occurs simultaneously with the onset of the BS. Cooling to $2^{\circ} \mathrm{C}$ substantially delayed the onset of LR but at the time of BS onset no increase of tension could be seen. This suggests that hypertonicity is a prerequisite for PRC to become apparent. The amplitude of PRC was in the order of 0.07 to $0.3 \mu \mathrm{N}$ (or $1 / 5000$ of the single twitch in $1 \mathrm{~T}$ Ringer). The increase in the interval between BS and LR by hypertonicity and cooling, together with the occurrence of PRC, strongly suggest that the BS is not an optical reflection of LR. This supports the hypothesis put forward earlier that the BS visualizes changes in myoplasmic $\mathrm{Ca}^{2+}$ activity by indicating conformational changes of the $\mathrm{Ca}^{i 2+}$ activated SR-ATPase and therefore can be used as an intrinsic $\mathrm{Ca}^{2+}$ release indicator [Oetliker, ]. Physiol., Lond. 305, 26-7P (1980)].
In vitro comparative studies of the dihydropyridine calcium-entry activators YC-170, CGP 28392 and BAY K 8644

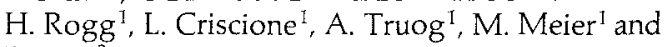
P. Erne

'Biological Research Department, Ciba-Geigy Ltd, CH-4002 Basel, Switzerland

${ }^{2}$ Department of Research, University Hospital, CH-4031 Basel, Switzerland

Recently, the novel dihydropyridine derivatives YC-170 (YC), CGP 28392 (CGP) and BAY K 8644: (BAY) have been reported to act in the opposite way to dihydropyridine $\mathrm{Ca}^{2+}$-entry blockers [Takenaka et al., Jap. J. Pharmacol., 32, Suppl. 139P' (1982); Truog et al., Calcium in Biological Systems, New York, Plenum Press (in press); Schramm et al. Nature, Lond. 303, 535 (1983)]. We have found that these compounds inhibit the binding of $\left[{ }^{3} \mathrm{H}\right]$ nitrendipine, a dihydropyridine derivative used for labelling putative $\mathrm{Ca}^{2+}$ channels, on guinea-pig heart membranes $\left(K_{\mathrm{i}}\right.$ : $6 \mathrm{nM}$ BAY, $117 \mathrm{nMCGP}$ and $670 \mathrm{~nm} Y \mathrm{YC})$. Like those of nifedipine ( $\left.K_{\mathrm{i}} 1 \mathrm{nM}\right)$, the curves had slopes close to unity, indicating a competitive interaction at dihydropyridine-sensitive sites. Direct evidence of $\mathrm{Ca}^{2+}$-entry stimulating properties has been obtained for CGP. This compound caused a dose-dependent increase in intracellular free $\mathrm{Ca}^{2+}$ in human platelets, as measured with the Quin-2 $\mathrm{Ca}^{2+}$ indicator, with a semimaximally effective concentration of $2.2 \times 10^{-7} \mathrm{M}$, a similar value to that obtained in the $\left[{ }^{3} \mathrm{H}\right]$ nitrendipine-binding studies.

In isolated guinea-pig atria, these agents exerted positively inotropic effects similar in their potency ratio to those observed in the binding experiments (BAY 1, CGP 1: 17, YC 1:600). The maximum inotropic effects of BAY and CGP and of $Y C$ corresponded respectively to $2 / 3$ and $1 / 3$ of those induced by isoprenaline or extracellular $\mathrm{Ca}^{2+}$. In guinea-pig right atria both BAY and CGP exerted direct positively chronotropic effects, as expected for $\mathrm{Ca}^{2+}$-influx activators. However, $\mathrm{YC}$ showed only negatively chronotropic activity, an effect typical of $\mathrm{Ca}^{2+}$-entry inhibition. In the isolated rat mesenteric bed perfused with a depolarizing solution, vasoconstrictor $\mathrm{Ca}^{2+}$ dose-response curves were shifted to the right by nifedipine $(0.3-30 \mathrm{nM})$ in a dose-dependent manner. By contrast, BAY and CGP, caused a distinct leftward shift of the $\mathrm{Ca}^{2+}$-dose-response curves at concentrations of 3-300 nM and 30-300 nM, respectively, and YC a marginal leftward shift at concentrations of 
200-2000 nM, i.e. similar ranges to those inducing inhibitory effects on $\left[{ }^{3} \mathrm{H}\right]$ nitrendipine binding. At higher concentrations, all three compounds produced $\mathrm{Ca}^{2+}$-antagonistic effects. These results indicate that the compounds act at dihydropyidinesensitive sites and exert partial agonistic activities in vascular and myocardial tissue.

\section{Frequency-dependent effects of} potassium-channel blockers on slow action potentials in the ventricular myocardium K. Schüttler, W. Boldt and B. Nilius Julius Bernstein Institute for Physiology, Martin Luther University, Leninallee 6, 4020 Halle (Saale), G.D.R.

In the literature it is often pointed out that slow action potentials (SAP) may be contaminated by outward currents and therefore the maximum rate of rise of SAP $\left(\dot{V}_{\text {max }}\right)$ as a measure of the slow inward current $i_{\mathrm{si}}$ should be used with caution.

By means of a conventional microelectrode technique, SAP and, via electronic differentiation, the first derivative of its upstroke were registered from isolated papillary muscles of the right ventricle of the rabbit heart. Preparations were bathed in $18 \mathrm{mmol} 1^{-1} \mathrm{~K}_{0}$ solution and the influence of the $\mathrm{K}^{+}$-channel blockers $\mathrm{CsCl}(20 \mathrm{mmol}$ $\left.1^{-1}\right), 4$-aminopyridine $\left(5 \mathrm{mmol} 1^{-1}\right)$ and $\mathrm{BaCl}_{2}$ $\left(0.8 \mathrm{mmol} \mathrm{l}^{-1}\right)$ was tested in a range of intervals of steady-state stimulation $\left(L_{0}\right)$ from 0.5 to $60 \mathrm{~s}$. In control conditions, the duration of SAP (SAPD) was moderately prolonged and $\dot{V}_{\max }$ distinctly increased towards long $L_{\mathrm{o}}$. Under the influence of the $\mathrm{K}^{+}$-channel blockers mentioned above, the magnitude of $\dot{V}_{\max }$ and its frequency-pattern were nearly unchanged in comparison to control while the SAPD was strongly prolonged in a frequency-dependent manner, increasing as $L_{0}$ increases

This suggests that the contribution of outward currents to SAP seems to be small therefore depressing effect of them on $\dot{V}_{\max }$ also. Unfortunately, an increase in $V_{\max }$ may be antagonized by the reduction of the resting potential by two of the blockers used $\left(\mathrm{CsCl}, \mathrm{BaCl}_{2}\right)$.

The pattern of the SAPD is produced by the rate-dependent lowered slope of the plateau phase while the slope of the terminal repolarization was reduced rate-independently. The inhibition of a partially voltage-inactivated transient outward current might be involved in it.

\author{
Morphological changes in the sarcoplasmic \\ reticulum of skeletal muscle during \\ excitation-contraction coupling \\ J. R. Sommer and N. Nassar \\ Duke University Medical Center, Durham, \\ NC 27710, U.S.A.
}

Excitation-contraction coupling (ECC) in striated muscle might be associated with visible morphological changes in the SR and/or the sarcolemma. It has been suggested that such alterations take place in the junctional processes (JP) of the junctional SR (JSR) which are thought to be directly involved in the translation of the action potential into $\mathrm{Ca}^{2+}$ release from the ISR. Moreover, it has been proposed that a reversible collapse of the SR membranes may play a role in ECC. The quick-freezing technique offers the unique opportunity literally to freeze events progressing at very high rates and to study their morphological manifestations. Intact single fibres $(R$. temporaria) were examined after quick-freezing at known intervals following stimulation and subsequent freeze-substitution or freezefracture. Quick-freezing was done on a liquid He-cooled copper block (Polaron) and freezed substitution in $-80^{\circ} \mathrm{C}$ acetone/osmium followed by postfixation in osmium/0.1 cacodylate at room temperature. Intervals between stimulation and impact on the copper block were measured with a digital microsecond clock. Ice crystal-free zones up to $12 \mu \mathrm{m}$ but usually from 1 to $5 \mu \mathrm{m}$ were obtained. Structural preservation was not strikingly different from glutaraldehyde-fixed tissue except for the following: the JSR contained electron-lucent regions, the core cylinders, and the granular material in the JSR was often arranged in rosettes and always separated from the JSR envelope by an electron-lucent halo. The SR showed very few regions of collapse. Their occurrence was not related to contractile activation. In tetanized fibres the electron density of the granular material in the SR was very much reduced over that in unstimulated fibres and the core cylinder was often obscured because of reduced contrast. Crossbridges could easily be seen. Freeze-fracture preparations showed smooth E-faces and particulate P-faces. After freeze-etching very few deep pits about $15 \mathrm{~nm}$ in diameter were seen in the Efaces of the JSR of unstimulated and tetanic fibres, as well as about $10 \mathrm{~ms}$ after stimulation. In several preparations frozen within the first millisecond after stimulation, however, both the JSR and the free SR were studded with such pits. Complementary particles were never observed. The occurrence of the pits during the first millisecond of ECC 
correlates with the beginning of the early large birefringence signal after stimulation. The technique described above together with $X$-ray microanalysis is expected to reveal changes in the spatial distribution of $\mathrm{Ca}^{2+}$ during a single contraction relaxation cycle of a muscle fibre.

\section{Electrogenicity of $\mathrm{Na}-\mathrm{Ca}$ exchange and its alteration by antiarrhythmic agents}

G. Vássory, D. Mentrard, R. Fischmeister, C. Lauer and R. Ventura-Clapier

Laboratoire de Physiologie Cellulaire Cardiaque, U-241 INSERM, Université Paris XI-91405 Orsay,

France

A Na-Ca exchange mechanism has been described in heart cells (Reuter \& Seitz, 1968). Such a countertransport which could exchange three or more $\mathrm{Na}$ for one $\mathrm{Ca}$ was reported to be voltagedependent and possibly electrogenic (Horackova and Vassort, 1979). Recently, Cs-loading of frog heart cells together with the presence of external Cs (K-free) have been shown to abolish both the inward and outward rectifications thus making it possible to analyse precisely the remaining currents (Mentrard et al., 1984). On applying a Na-poor solution $(39.2 \mathrm{mM})$, under voltage clamp conditions on frog atrial preparations, an outward current was generated during both depolarizations and hyperpolarizations around the resting membrane potential. The current amplitude was sensitive to the external $\mathrm{Ca}$ and described a U-shaped function of the membrane potential. Such a relation could be expected for the exchange current $I_{\mathrm{ex}}$, if one considers following Mullins' model (1977), that $I_{\mathrm{ex}}$ is a hyperbolic sinus function of $E_{\mathrm{m}}-E_{\mathrm{ex}}$ ( $E_{\mathrm{ex}}$ the equilibrium potential of the exchange $=2$ $E_{\mathrm{Na}}-E_{\mathrm{Ca}}$ assuming a 4 to $\mathrm{I}$ stoichiometry). $\mathrm{Na}_{0}$ alterations did not induce variations in $I_{\mathrm{ex}}$ in the presence of $\mathrm{La}^{3+}, \mathrm{Co}^{2+}$. Variations in $I_{\mathrm{ex}}$ were also reduced by the addition of several antiarrhythmic agents. Most of the local anaesthetics (antiarrhythmic of class I), but lidocaine, reduced $I_{\text {ex }}$ generally to a different extent than they reduced $I_{\mathrm{Ca}}$, the slow inward current. The effect of amiodarone (class III) were limited to $I_{\mathrm{Ca}}$ and might be related to cyclic AMP reduction since in whole frog hearts treated with aminophylline and propanolol, amiodarone $\left(2 \times 10^{-5} \mathrm{M}\right)$ decreased cAMP content by one third. Nifedipine has no effect on $I_{\text {ex }}$ but $D 600$ (class IV, $10^{-6} \mathrm{M}$ ) reduced both $I_{\mathrm{ex}}$ and $I_{\mathrm{Ca}}$.

Such an exchange current might be involved both in the rhythmic activity of cardiac cells since, on the mathematical model, $I_{\text {ex }}$ was shown to be of particular importance in controlling AP duration and re-excitability (Fischmeister \& Vassort, 1981) and in the negative inotropic and chronotropic effects of some antiarrhythmic agents.

\section{Action potentials of fast and slow fibres in rat skeletal muscles}

Willemien Wallinga-de Jonge ${ }^{\mathrm{I}}$, Paul de Jong ${ }^{1}$, Bert Albers ${ }^{1}$ and Peter Wirtz ${ }^{2}$

Bio-information Group, Twente University of

Technology, P.O. Box 217, 7500 AE Enschede,

The Netherlands

${ }^{2}$ Department of Cytology and Histology,

University of Nijmegen, The Netherlands

In this study single motor units were stimulated. It was possible to insert a micropipette in some fibres of the stimulated motor unit. Motor units of the soleus were indicated by the twitch contraction time. To determine the motor unit type in EDL the twitch contraction time and the histochemical characteristics were used. At the end of the experiment the fibres of the EDL motor unit were therefore glycogen depleted following the known method of Kugelberg. The intracellular recordings presented concern slow soleus and FTG (fast twitch glycolytic) EDL fibres. During the experiment the electromyogram and the force of the motor unit was recorded continuously to verify that the same motor unit was active.

The intracellular measurements were done with micropipettes. To avoid movement artefacts the micropipettes had a long flexible taper (a tip diameter about $0.2 \mu \mathrm{m}$; impedance $<55 \mathrm{M} \Omega$ ). The pipettes were connected to a microprobe system of W.P.I. (mode M-707) by means of an $\mathrm{Ag} / \mathrm{AgCl}$ electrode. The frequency band width of the system was checked in each penetrated fibre before the motor unit was stimulated. The intracellular recordings (digitally stored on disc; sample frequency $100 \mathrm{kHz}$ ) of slow and FTG fibres were compared using a set of characteristics.

Intracellular action potentials of slow and FTG fibres differed significantly in the mean values of all but one characteristic. The slow fibre action potentials showed less negative membrane potentials, smaller amplitudes and lower rates in the depolarization and repolarization phases in comparison with FTG fibres. The time courses of the intracellular action potentials of slow fibres are slow with respect to those of FTG fibres.

The differences described will influence the extracellular single action potential and consequently the motor unit action potential. In this way the results described contribute to the fundamental knowledge of the generation of the 
electromyogram at single fibre and motor unit level.

\section{Conductance kinetics and selectivity of sodium channels expressed in tonic muscle fibres of the frog after denervation \\ J. Zachar, D. Zacharová, M. Henček and \\ G. A. Nasledov \\ Centre of Physiological Sciences, Slovak Academy \\ of Sciences, Vlárska 5, 83306 Bratislava, \\ Czechoslovakia}

The aim of the experiments was to characterize the new sodium channels in the muscle membrane of slow (tonic) muscle fibres of the frog, which appear only after denervation. The conductance kinetics parameters and selectivity of $\mathrm{Na}$ channels were compared with those in normal and denervated twitch muscle fibres from the same muscle. The measurements were performed under voltageclamp conditions in cut muscle fibre segments in potassium-free internal and external environment. Sodium conductances $\left(G_{\mathrm{Na}}\right)$ as functions of both time and voltage were found to be described quantitatively by the Hodgkin-Huxley model for the sodium channel $\left(G_{\mathrm{Na}}=\bar{G}_{\mathrm{Na}} m^{3} h\right)$ with $\bar{G}_{\mathrm{Na}}=10 \mathrm{mS} \mathrm{cm}-2$. The new sodium channels in tonic muscle fibres show slower kinetics in comparison with $\mathrm{Na}$ channels in normal phasic fibres $\left(\bar{\alpha}_{m}, \bar{\beta}_{m}, \bar{\beta}_{h}\right)$. The other kinetic parameters are fairly similar in both the twitch and the slow muscle membranes. The selectivity of sodium channeis appearing after denervation in tonic muscle fibres is very close to the selectivity of $\mathrm{Na}$ channels in normal twitch fibres from the same muscle $\left(P_{\mathrm{Na}}: P_{\mathrm{Li}}: P_{\mathrm{NH}_{4}}: P_{\mathrm{K}}=1: 0.88: 0.23: 0.012\right)$. The experiments represent a further positive test of the hypothesis stating that the new sodium channels are of the same genetic origin as the sodium channels present in twitch muscle fibres under physiological conditions. Synthesis of sodium channel proteins in innervated tonic fibres is suppressed by the action of innervating nerve cells. When this inhibitory effect is released by denervation the genetic information is expressed and new sodium channels become formed.

\section{REGULATION IN SMOOTH MUSCLE AND SKELETAL MUSCLE}

\section{Multinuclear magnetic resonance study of smooth muscle regulation \\ Heather A. Cole ${ }^{1}$, Barry A. Levine ${ }^{2}$, Valerie B. \\ Patchell ${ }^{A}$ and S. Victor Perry ${ }^{1}$ \\ ${ }^{1}$ Department of Biochemistry, University of \\ Birmingham, Birmingham B15 2TT \\ ${ }^{2}$ Inorganic Chemistry Laboratory, University of Oxford, Oxford OXI $3 Q R, U . K$.}

A considerable body of evidence indicates that phosphorylation of the P-light chains of myosin results in a significant increase of the $\mathrm{Mg}^{2+}$ activated ATPase of vertebrate smooth muscle actomyosin. We are using ${ }^{31} \mathrm{P}$ and ${ }^{1} \mathrm{H}$ n.m.r. to study the structural aspects of phosphorylation of the P-light chain in purified chicken gizzard myosin as well as for the isolated light chain. Using fully phosphorylated, highly active myosin [Cole et al., FEBS Lett. 158, 17-20 (1983)] we are able to resolve and identify the ${ }^{31} \mathrm{P}$ signal deriving from the phosphoserine residue of the P-light chain at smooth myosin concentrations in the range 4-8 $\mu \mathrm{M}$. The spectral characteristics of the signal (resonance energy and relaxation parameters) have been followed under varying solution conditions and at different temperatures in order to follow effects of $\mathrm{pH}, \mathrm{Mg}^{2+}$, nucleotides and urea. The hydrolysis of ATP can be followed simultaneously by monitoring the ${ }^{31} \mathrm{P}$ signals of ATP, ADP and inorganic phosphate. Analysis of the lineshapes of these resonances and preliminary data obtained with isolated myosin subfragment-1 provide kinetic information about product dissociation which " reflects structural features of the enzyme-nucleotide interaction.

Complex formation with F-actin led to marked perturbation of the P-light chain signal of smooth myosin, in contrast to the spectral effects observed in the presence of G-actin. Studies on the isolated light chain, phosphorylated and dephosphorylated, by ${ }^{31} \mathrm{P}$ and ${ }^{1} \mathrm{H}$ n.m.r. show that complex formation with actin does not occur. It is likely therefore that the phosphorylation of the P-light chain enhances the $\mathrm{Mg}^{2+}$-activated ATPase by altering the configuration of the domains of the myosin head. Studies are under way to define the disposition of the P-light chain relative to the myosin active site and the surfaces of contact with actin at different smooth myosin phosphorylation levels. These 
studies should shed light on the nature of the correlation between the extent of phosphorylation and the ATPase activity. (This work is supported by the M.R.C.).

\section{Phosphorylation of the $P$ light chain of chicken gizzard myosin}

H. A. Cole, H. S. Griffiths, V. B. Patchell and

S. V. Perry

Department of Biochemistry, University of

Birmingham, P.O. Box 363, Birmingham B15 2TT,

U.K.

In certain preparations of chicken gizzard myosin, the $P$ light chain was observed to migrate as three bands on electrophoresis in $6 \mathrm{M}$ urea. Despite extensive investigations, no evidence was obtained for the presence of isoforms of the unphosphorylated $P$ light chain of gizzard myosin as has been reported for slow skeletal and cardiac muscles. The specific radioactivity and the mobilities on two-dimensional isoelectric focusing gels after incubation with [ $\gamma_{-}{ }^{32}$ P]ATP were such to suggest that the two most acidic bands represented mono- and diphosphorylated forms of the P light chain. On incubation of the myosin preparation with ATP with or without added purified skeletal or smooth muscle light chain kinase preparations, the diphosphorylated form increased in amount at the expense of the dephosphorylated and monophosphorylated forms.

On incubation of isolated dephosphorylated $\mathrm{P}$ light chains of gizzard myosin with a purified rabbit skeletal muscle light chain kinase, the monophosphorylated form of the $\mathrm{P}$ light chain was produced rapidly and then converted into the diphosphorylated form more slowly. Study of autoradiograms of two-dimensional electropherograms of tryptic digests of the $P$ light chains that had been previously incubated with skeletal light chain kinase and $\left[\gamma_{-}{ }^{32} \mathrm{P}\right] \mathrm{ATP}$ suggested that two sites on the $P$ light chain were phosphorylated. The role of phosphorylation of the two sites in the activation of the $\mathrm{Mg}^{2+}$-ATPase of gizzard actomyosin is as yet uncertain. Nevertheless the difference in the phosphorylation rates of the two sites, which appear to be phosphorylated by the same kinase, needs to be taken into consideration in experiments concerned with possible cooperative effects during phosphorylation of the two heads of myosin.
Photoaffinity labelling of skeletal muscle myosin light chain kinase with $8 \mathrm{~N}_{3}$-ATP indicates two nucleotide binding sites

U. Geuss ${ }^{1}$, H.-J. Schäfer ${ }^{2}$, G. W. Mayr ${ }^{1}$ and L. M. G. Heilmeyer $\mathrm{Jr}^{1}$

${ }^{1}$ Institut für Physiologische Chemie I,

Ruhr-Universität, Universitätsstraße 150,

4630 Bochum, G.F.R.

${ }^{2}$ Institut für Biochemie der

Johannes-Gutenberg-Universität,

Johann-Joachim-Becherweg 30, 6500 Mainz,

G.F.R.

Skeletal muscle myosin light chain kinase has a head-tail structure; the head contains the catalytic, the light chain 2, and the calmodulin binding domains; the function of the tail is unknown. We use the ATP photoaffinity analogue, $8 \mathrm{~N}_{3}$-ATP, to localize the catalytic domain. $8 \mathrm{~N}_{3}$-ATP is essentially no substrate for MLCK; however, it significantly inhibits the MLCK activity. A mixed-type inhibition pattern with a $K_{\mathrm{I}}$ value of $0.25 \mathrm{~mm}$ is found suggesting minimally two $8 N_{3}$-ATP binding sites to be involved.

Half-maximal photoincorporation of $8 \mathrm{~N}_{3}$-ATP is obtained at about $0.45 \mathrm{~mm}$. A maximum of $1.4 \mathrm{~mol} 8 \mathrm{~N}_{3}$-ATP is incorporated per mol MLCK. Parallel to the label incorporation, the MLCK activity decreases by up to $40 \%$. Without irradiation no incorporation and no irreversible loss of activity is observed. Magnesium is essential for the photoincorporation, the presence of $\mathrm{Ca}^{2+}$-calmodulin has no effect.

The photoincorporation decreases by $40 \%$ at saturating concentrations $(10 \mathrm{~mm})$ of either MgATP or MgADP under the same conditions; a combination of both nucleotides results in a further $20 \%$ decrease of label incorporation. Twenty millimolar adenosine has no effect.

The data suggest that besides the ATP binding site an additional, nucleotide binding site may exist on MLCK which also can bind $8 \mathrm{~N}_{3}$-ATP.

\section{Comparison of latch-state in skinned taenia coli smooth muscle from guinea-pig and} catch-state in skinned anterior byssus retractor muscle (ABRM) or Mytilus edulis

M. Gagelmann and K. Güth

II. Physiologisches Institut, Universität

Heidelberg, Im Neuenheimer Feld 326,

D-6900 Heidelberg, G.F.R.

Depending on the choice of hypothesis the tension maintenance without energy consumption (catch) in the ABRM is thought to be due to slow breaking actomyosin crossbridges and/or associated 
with the protein paramyosin. A similar contraction reminder like a catch-state, called latch, is observed in skinned taenia coli smooth muscle fibres. In both muscles, removal of $\mathrm{Ca}^{2+}$ is considered to be a prerequisite for the initiation of catch or latch: when $\mathrm{Ca}^{2}+$ is withdrawn the ATPaseactivity rapidly drops to resting levels while tension is still maintained. The catch is abolished by cAMP and the latch by addition of inorganic phosphate $\left(\mathrm{P}_{\mathrm{i}}\right)$. To find out whether the regulation of catch and/or latch is exclusively controlled by $\mathrm{Ca}^{2+}$ we investigated if a similar tension maintenance is also observed in the presence of $\mathrm{Ca}^{2+}$. By lowering the ionic strength during contraction in taenia coli smooth muscle the force slowly decreases or even stays constant while the ATPase activity is strongly depressed and isotonic shortening is inhibited (phosphorylation of the regulatory myosin light chains is not altered). Since addition of $P_{i}$ causes a change of tension to a lower level this state may be a latch-state. On the other hand, in the contracted ABRM a lowering of the ionic strength does not initiate a catch-like state: isotonic shortening is not influenced under similar conditions described for taenia coli and isotonic shortening at low ionic strength is not significantly accelerated by cAMP.

The experiments suggest that the latch-state in taenia coli depends on attached or slowly cycling crossbridges and that its regulation is not exclusively dependent on the $\mathrm{Ca}^{2+}$ concentration while it could be influenced by a step that involves binding of $\mathrm{P}_{\mathrm{i}}$ to myosin. In contrast, in the ABRM a CAMP sensitive catch-like state is initiated when $\mathrm{Ca}^{2+}$ is lowered. The participation of paramyosin in regulation of the catch is still controversial: while isolated paramyosin is a substrate for the cAMP dependent protein kinase, a cAMPdependent phosphorylation of this protein in skinned fibre preparations is not observed. (Supported by the Deutsche Forschungsgemeinschaft.)

\section{A slow calcium-dependent process precedes force development, phosphorylation and ATPase activity in skinned taenia coli \\ K. Güth ${ }^{1}$ and U. Mrwa ${ }^{2}$ \\ II. Physiologisches Institut der Universität. Im Neuenheimer Feld 326, D-6900 Heidelberg, G.F.R. \\ ${ }^{2}$ Dr. Karl Thomae GmbH, D-7950 Biberach, G.F.R.} Skinned taenia coli is activated either by increasing the calcium-concentration in the presence of ATP or by increasing the ATP concentration at constant calcium concentration. The force develops slower and after a delay when the preparation is activated by increasing the calcium concentration (at constant ATP concentration). The rate of force generation is increased and the delay vanishes when the muscle is activated by adding ATP after the muscle was preincubated in an ATP-free but calciumcontaining solution. The myosin phosphorylation is low before the force is generated but increases rapidly after activation. The ATPase activity of the preparation precedes the force development but is nevertheless delayed when the fibre is activated by increasing the calcium concentration. In contrast, the ATPase activity increases without delay when the contraction is started by adding ATP after preincubation in an ATP-free but calciumcontaining solution. It is concluded that a slow, calcium-dependent process precedes the activation of the skinned smooth muscle preparation. This process is a necessary precondition for the myosin phosphorylation, the development of force and the actomyosin ATPase activity of the muscle.

\section{Myosin light chain phosphorylation in intact uterine muscle: effect of contracting and relaxing agents}

S. Harbon ${ }^{1}$, L. Dokhac ${ }^{1}$, C. Janmot ${ }^{2}$ and

A. d'Albis ${ }^{2}$

${ }^{1}$ Endocrinologie et Régulation Cellulaire et

${ }^{2}$ Biologie Physicochimique, Université Paris-Sud, 91405 Orsay, France

It is generally agreed that contraction of smooth muscles is linked to the activation of the actomyosin ATPase activity by the phosphorylation of one type of the light chain (LC) of myosin. We have tested this hypothesis in the oestrogenprimed rat uterus. Myometrial strips were exposed to various treatments, isometric contraction was measured, and the extent of light chain phosphorylation was determined after rapid freezing in liquid nitrogen. Two dimensional electrophoresis revealed five spots having the same molecular weight $(20000)$ as the light chain, with a pI between 5.15 and 4.95. Two of these spots (pI 5.09 and 5.00) were not present in pure uterine myosin, whether prepared from incubated or nonincubated tissue; they do not represent light chain isoforms but rather degradation products appearing during the treatment. Two spots ( $\mathrm{pI} 5.15$ and 5.06) were respectively identified as the non-P and the $P$-forms of the $\mathrm{LC}$; their relative amounts were used to calculate the extent of phosphorylation. The fifth spot ( $\mathrm{pI}$ 4.95) may represent a disphosphorylated myosin species; it consistently averaged less than $5 \%$ of the total. Myometrial strips 
incubated in a normal $\mathrm{Ca}^{2+}$-medium $(0.8 \mathrm{~mm})$ exhibited basal contractions and an incorporation of $0.2 \mathrm{~mol} \mathrm{P} / \mathrm{mol} \mathrm{LC}$. Removal of $\mathrm{Ca}^{2+}$ resulted in almost complete dephosphorylation, coincident with a total relaxation of the muscle. Exposure of the myometrium to carbachol caused tetanic contractions with an increase to $0.5 \mathrm{~mol} \mathrm{P} / \mathrm{mol}$ LC; addition of atropine to carbachol-contracted myometrium decreased both the myosin- $\mathrm{P}$ content and tension to resting levels. $\beta$-adrenergic agonists, which elevate intracellular cAMP, induce uterine relaxation. Addition of isoproterenol to a resting myometrium caused a slight but significant decrease in myosin-P; also its addition prior to carbachol markedly prevented the increase in myosin phosphorylation normally induced by the cholinergic effector. Forskolin $(1 \mu \mathrm{M})$ increased intracellular CAMP, caused relaxation and a concomitant decrease in basal myosin-P. The data imply that (1) myosin LC phosphorylation, regulated by $\mathrm{Ca}^{2+}$-dependent mechanisms, is essential for uterine contractions and (2) dephosphorylation whether mediated by $\mathrm{Ca}^{2+}$ deprivation or by cAMP may be essential for uterine relaxation. However an exclusive role for cAMP in mediating relaxation and myosin dephosphorylation in intact myometrium was questioned: prostaglandin $E_{2}$-induced contractions were accompanied by the expected increase in P-incorporation into LC despite the concomitant elevations in intracellular CAMP.

\section{Myosin-adenine-nucleotide binding and light chain phosphorylation in rat skeletal muscles}

\section{S. Hebisch, H. Sies and S. Soboll}

Institut für Physiologische Chemie I, Universität

Düsseldorf, D-4000 Düsseldorf, G.F.R.

In fast and slow skeletal muscles of the rat in vivo adenine-nucleotide binding to myosin is fibre-type specific. Resting fast-twitch muscles contain predominantly myosin-ATP complexes whereas resting slow-twitch muscles contain predominantly myosin-ADP complexes [Hebisch et al., Biochim. Biophys. Acta 764, 117 (1984)]. This is due to a difference in innervation. While during $15 \mathrm{~min}$ of denervation the content of myosin-bound ADP decreased from about 0.9 to $0.3 \mathrm{nmol} \mathrm{mg}$ protein $^{-1}$ in slow-twitch muscles, it remained constant $\left(0.2 \mathrm{nmol} \mathrm{mg}\right.$ protein $\left.{ }^{-1}\right)$ in fast-twitch muscles. During short-term stimulation $(6 \mathrm{~s})$ of fast-twitch muscles the content of myosin-bound ADP increased with force and filament overlap, reaching a maximum of about $1.3 \mathrm{nmol} \mathrm{mg}$ protein $^{-1}$. This indicates that myosin-bound ADP can serve as a measure of the steady-state amount of force-producing actomyosin complexes. During longer working periods $(\geqslant 10 \mathrm{~s})$ the binding properties of myosin change reversibly from an ATP - to an ADP-binding state, associated by a substantial increase in myosin light chain phosphorylation. In slow-twitch muscles, however, the content of myosin-bound ADP remained unchanged during stimulation.

Our results indicate that during prolonged stimulation of fast-twitch muscles the binding properties of myosin change towards that of the slow-twitch type [Hebisch et al., Biochim. Biophys. Acta (in press)]. It seems reasonable to propose that the changes in myosin-adenine-nucleotide binding described here reflect a mechanism of longterm regulation in fast-twitch muscles. However, the causal relationship to myosin light chain phosphorylation remains to be established.

\section{The role of actin modulators in smooth and skeletal muscle}

Horst Hinssen, Frank E. Engels and J. Victor Small Institute of Molecular Biology, Austrian Academy of Sciences, Salzburg, Austria

$\mathrm{A} \mathrm{Ca}^{2+}$-dependent actin modulator has been purified from pig stomach smooth muscle [Hinssen et al. FEBS Lett. 166, 90-5 (1984)]. The interaction of this protein (PSAM) with actin and its effect on the actin polymer state have been studied in detail. The modulator, similar to gelsolin from nonmuscle cells, binds to both $\mathrm{G}$ - and $\mathrm{F}$-actin in the presence of $\mathrm{Ca}^{2+}>10^{-6} \mathrm{M}$. It acts as a potent nucleus for actin polymerization, caps the fast polymerizing end of F-actin and severs actin filaments. The latter effect is partly inhibited by tropomyosin. Each PSAM molecule binds $\mathrm{Ca}^{2+}$ and two molecules of actin and the stable $2: 1$ complex formed is still effective as a nucleus for polymerization and capping. However, it has no more severing activity and is practically $\mathrm{Ca}^{2+}$-insensitive.

Corresponding actin-modulating proteins have now been isolated and purified from chicken gizzard smooth muscle and rabbit skeletal muscle, indicating that proteins of this type are generally present in muscle tissue. All three modulators were found to be functionally identical with regard to their interaction with actin but reveal differences in molecular weight, distribution of spots in two-dimensional electrophoresis, and peptide maps after limited proteolytic digests. The modulators were identified as true constituents of the muscle cells and the myofibrils, respectively, by 
two dimensional electrophoresis and immunoblotting of two-dimensional electropherograms using affinity purified aPSAM. When applied to isolated smooth muscle cells and striated muscle myofibrils a-PSAM showed a distribution similar to that of $a$-actinin, namely a spotty distribution in smooth muscle cells, taken as coincident with the cytoplasmic dense bodies and a Z-line localization in skeletal muscle. These results indicate that one of the functions of the modulators in the muscle cells is that of an actin ending factor at intracellular attachment sites.

\section{Isometric tension and phosphorylation of the 20000 regulatory myosin light chain (LC) and satellite proteins in absence of calcium in chemically skinned smooth muscle fibres from taenia coli \\ T. Kossmann, M. Gagelmann and J. C. Rüegg \\ II. Physiologisches Institut, Universität \\ Heidelberg, Im Neuenheimer Feld 326, \\ D-6900 Heidelberg, G.F.R.}

In fibres incubated in relaxing solution $\left(\mathrm{Ca}^{2+}<10^{-8} \mathrm{M} ; \mathrm{pH} 6.7\right)$ an increase of the temperature from $22^{\circ} \mathrm{C}$ to $37^{\circ} \mathrm{C}$ causes a slow increasing contractile force. Within $5 \mathrm{~h}$ the tension increases to a plateau where $42.5 \pm 6.6 \%$ S.E.M. $(n=6)$ of the tension is obtained with $4 \times 10^{-5} \mathrm{M}$ $\mathrm{Ca}^{2+}$. As phosphorylation of the $\mathrm{LC}$ by a $\mathrm{Ca}^{2+}$ and calmodulin-dependent myosin light chain kinase (MLCK) is considered to be a key event in smooth muscle activation LC phosphorylation was analysed by two-dimensional gel electrophoresis. The content of unphosphorylated LC (U-LC) in fibres incubated only in relaxing solution is $83.2 \pm 1.1 \%(n=6)$ of total light chains. After 30 min contraction with $4 \times 10^{-5} \mathrm{M} \mathrm{Ca}^{2+}$ at $22^{\circ} \mathrm{C}$, in six fibres U-LC decreases to $36.8 \pm 4.1 \%$. Relaxation of the fibres $(n=6)$ by removal of $\mathrm{Ca}^{2+}$ is again associated with a dephosphorylation of the phosphorylated LC (P-LC) from 63.2 $\pm 4.1 \%$ to $14.4 \pm 2.2 \%$. A different composition of LC is observed after further incubation for $5 \mathrm{~h}$ at $37^{\circ} \mathrm{C}$ in the absence of $\mathrm{Ca}^{2+}$ : U-LC $19.7 \pm 2.6 \%, \mathrm{P}-\mathrm{LC}$ $35.4 \pm 2.6 \%$ and $44.5 \pm 3.0 \%$ phosphorylated satellite proteins of the $20000 \mathrm{LC}(n=6)$. The fact that the tension increase at $37^{\circ} \mathrm{C}$ is accompanied by a LC phosphorylation suggests that the MLCK has lost its $\mathrm{Ca}^{2+}$ sensitivity. This is supported by experiments with homogenates from fresh fibres and fibres treated at $37^{\circ} \mathrm{C}$ and tested on isolated LC from bovine heart as substrate. Phosphate incorporation into LC was calcium dependent in homogenates from fresh fibres but in fibres incubated at $37^{\circ} \mathrm{C}$ (for $5 \mathrm{~h}$ ) phosphate incorporation occurred even in absence of $\mathrm{Ca}^{2+}$. The loss of $\mathrm{Ca}^{2+}$ regulation of MLCK activity and force production could not be prevented by the addition of the protease inhibitor leupeptin $\left(10^{-4} \mathrm{M}\right)$. Treatment with a specific LC-phosphatase from bovine aorta [DiSalvo et al., Biochem. Biophys. Res. Commun. 3, 906 (1983)] partly causes dephosphorylation of $\mathrm{P}-\mathrm{LC}$ and satellites. The fact that the composition of LC proteins in fibres incubated for $5 \mathrm{~h}$ at $37^{\circ} \mathrm{C}$, in the absence of $\mathrm{Ca}^{2+}$, is not altered by exposure to $4 \times 10^{-5} \mathrm{M} \mathrm{Ca}^{2+}$ while tension rises further, suggests that in smooth muscle, pathways other than phosphorylation may exist for activation of contraction. (Supported by the Deutsche Forschungsgemeinschaft.)

\section{Supramolecular regulation of actin-activated $\mathrm{Mg}^{2+}$-ATPase activity of Acanthamoeba myosin II by phosphorylation of its heavy chain J. Kuznicki* and E. D. Korn Laboratory of Cell Biology, NIH, Bldg. 3,} Rm. B1-22, Bethesda, Maryland, 20205, U.S.A.

Myosin II is a typical two-headed myosin consisting of a pair of heavy chains and two pairs of light chains. Myosin II has high actin-activated ATPase activity when its three regulatory serines, located at the C-terminal end of the heavy chain, are dephosphorylated. The aim of this study is to understand how the level of phosphorylation at the end of the tail regulates the catalytic site located on the myosin head.

Under the conditions of the ATPase assay dephosphorylated myosin II is always filamentous. The size of the filaments varies depending on the conditions, but there is no correlation between filament size and the actin-activated ATPase activity. When the C-terminal portion of the heavy chain that contains the regulatory serines is proteolytically removed by chymotrypsin, myosin II loses its ability to form bipolar filaments. This is accompanied by complete loss of its actinactivated ATPase activity under standard assay conditions.

Phosphorylated myosin II forms smaller filaments than dephosphorylated myosin II, and its filaments have no actin-activated ATPase activity under conditions that are optimal for dephosphorylated myosin II. Phosphorylated myosin II inhibits the actin-activated ATPase activity of dephosphorylated myosin II when they are present

*Present address: Nencki Institute of Experimental Biology, 3 Pasteur, 02-093 Warsaw, Poland. 
in the same filament, but not when they are mixed as separate homopolymers. Limited digestion of myosin II by trypsin selectively cleaves the heavy chain at one site, but the proteolytic products remain associated and form bipolar filaments under conditions of enzymatic assay. Dephosphorylated trypsin-cleaved myosin II has no actin-activated ATPase activity. It behaves like phosphorylated myosin in copolymers with undigested dephosphorylated myosin II, inhibiting its actin-activated ATPase activity. Factors such as $\mathrm{Mg}^{2+}, \mathrm{KCl}, \mathrm{pH}$ or temperature that affect filament formation affect in a parallel manner the actin-activated ATPase activity of myosin II. On the basis of these data, we propose that regulation of actin-activated ATPase activity of myosin II occurs at the filament level and, moreover, that the actin-activatable ATPase activity of myosin II is expressed only when the myosin is filamentous.

\section{Identification of the $120000 \mathrm{M}_{\mathrm{r}}$ regulatory protein of smooth muscle thin filaments S. Marston and C. Smith \\ The Cardiothoracic Institute, 2 Beaumont Street, London W1N 2DX, U.K.}

Our work on sheep aorta vascular smooth muscle thin filaments has shown that they contain a $120000 M_{\mathrm{r}}$ inhibitory protein which is involved in the $\mathrm{Ca}^{2+}$-dependent regulation of the thin filaments' activity [Marston \& Smith, ]. Musc. Res. Cell Motility 5, 559-75 (1984); Marston et al., Trans. Biochem. Soc. (in press)] (see poster by Smith \& Marston). We have prepared thin filaments also from rabbit stomach and chicken gizzard smooth muscle by our established technique (Marston \& Smith, 1984). All thin filament preparations activated skeletal muscle myosin in a $\mathrm{Ca}^{2+}$-dependent way. (Maximum $\mathrm{Ca}^{2+}$ sensitivity: aorta: $5 \times$, stomach: $2.4 \times$, gizzard: $1.3 \times$.) All thin filament preparations contained mainly actin, tropomyosin and the $120000 M_{\mathrm{r}}$ protein (relative quantities, $\mathrm{w} / \mathrm{w}$, by gel scanning aorta $1: 0.40: 0.10$, stomach $1: 0.33: 0.09$, gizzard $1: 0.47: 0.085$ ). Thus the $120000 M_{\mathrm{r}}$ protein is probably a component of all smooth muscle thin filaments. What is its nature?

$\mathrm{Dr}$ K. Burridge of the University of North Carolina compared a sample of our $120000 M_{\mathrm{r}}$ protein with $a$-actinin and vinculin, two known high molecular weight actin-binding proteins. $120000 M_{\mathrm{r}}$ has a higher molecular weight on $7 \frac{1}{2} \%$ SDS gels and did not crossreact with antibodies to $a$-actinin or vinculin.
The apparent molecular weight of ' $120000 M_{r}^{\prime}$ ' protein on gel electrophoresis is from 120000 to 150000 depending on the molecular weight markers used. Many of the properties of ' 120000 $M_{\mathrm{r}}^{\prime}$ protein resemble Kakiuchi's caldesmon [Sobue et al., Biomed. Res. 3, 188-96 (1982)]. Dr Clive Sanders of the University of Alberta gave us a sample of gizzard caldesmon prepared as described by Ngai et al. [Ngai et al., Biochem. J. 218, 863-70 (1984)]. This protein was indistinguishable from

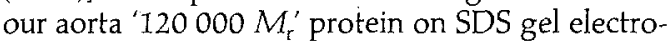
phoresis. Thus it seems quite likely that ' 120000 $M_{r}^{\prime}$ protein and 'caldesmon' are the same protein. If so, this regulatory protein plays a role in the control of thin filaments of both smooth and nonmuscle actomyosins [Kakiuchi et al, FEBS Lett. 154, 351-6 (1983)]. (Supported by British Heart Foundation.)

\section{The phosphorylation of human and baboon cardiac troponin I by $3^{\prime}, 5^{\prime}$-cAMP-dependent protein kinase}

\author{
A. J. G. Moir ${ }^{1}$ and S. V. Perry ${ }^{2}$ \\ ${ }^{1}$ Department of Biochemistry, University of \\ Sheffield, U.K. \\ ${ }^{2}$ Department of Biochemistry, University of \\ Birmingham, U.K.
}

Troponin I has been isolated from baboon and human cardiac muscle. The electrophoretic mobilities of the proteins in the presence of SDS were identical to that of rabbit cardiac troponin I. Incubation of baboon and human cardiac troponin I with $\left[\gamma_{-}{ }^{32} \mathrm{P}\right] \mathrm{ATP}$ and $3^{\prime}, 5^{\prime}$-cAMP-dependent protein kinase resulted in the incorporation of approximately 0.45 and $1.0 \mathrm{~mol}{ }^{32} \mathrm{P} / \mathrm{mol}$ troponin I respectively. In both cases, virtually all of the radioactivity was incorporated as o-phosphoserine. Tryptic digestion of the ${ }^{32} \mathrm{P}$-labelled proteins indicated that two sites of phosphorylation were present, a major site into which ${ }^{32} \mathrm{P}$ was incorporated relatively rapidly, and a minor site, the phos. phorylation of which was much slower. The major phosphorylation site has been identified within the sequence Arg-Ser-Asx-Arg-Ser-Tyr, which is homologous to residues $19-24$ of rabbit cardiac troponin I (Arg-Ser-Asx-Arg-Ala-Tyr), Ser 20 of which may be phosphorylated by protein kinase. We have no direct evidence as to which of the serines is phosphorylated in the baboon and human peptides, but it would seem probable that Ser 20 rather than Ser 23 was the site of phosphorylation. The ${ }^{32} \mathrm{P}$ content of the baboon 
peptide was significantly lower than $1 \mathrm{~mol} / \mathrm{mol}$ peptide, indicating that the serine was partially phosphorylated prior to incubation with protein kinase. The minor phosphorylation site is equivalent to serine 147 of rabbit cardiac troponin I; the amino-acid composition of the 14-residue peptide containing this site from baboon cardiac troponin I shows only one difference from that of the equivalent region of rabbit cardiac troponin I and would suggest the replacement of threonine 157 in the rabbit by alanine in the baboon. It is well documented that phosphorylation of troponin I occurs in the perfused rabbit and rat heart, and that this increases markedly in the presence of physiological concentrations of adrenergic compounds, due to enhanced phosphorylation of serine 20 . The observations that baboon and human cardiac troponin I contain a serine at an identical position to the phosphorylatable serine in rabbit troponin $\mathrm{I}$, and that this region of baboon and human cardiac troponin I may be phosphorylated by c-AMP-dependent protein kinase, would suggest that the response of the primate heart to catecholamines may be related to the level of phosphorylation of troponin I.

\section{The temperature dependency of changes in contractility during tonic activation of rat tracheal smooth muscle}

\section{U. Peiper, C.-F. Vahl, E. Donker, D. Buchholz and}

P. Benecke

University of Hamburg, Institute of Physiology,

Martinistrasse 52, 2000 Hamburg 20, G.F.R.

The term 'muscle contractility' should be used to describe the kinetics of crossbridge cycling. Previous experiments on smooth muscle have shown the effects of temperature changes and/or tonic activation durations on contractility. Contraction kinetics were studied in the isolated segment of the rat trachea by analysing tension recovery after the cessation of inhibiting length vibration $(100 \mathrm{~Hz}$, about $6 \%$ of the muscle length). The initial fast component reflects the reattachment kinetics of those crossbridges previously detached by the vibration. The slow component is affected by the kinetics of the regular actin-myosin interaction cycle. Minimum time constants reflect maximum contraction kinetics. The experiments were carried out at temperatures ranging from 25 to $40^{\circ} \mathrm{C}$.
Such force parameters as equilibrium tension (about $8.5 \mathrm{mN}$ at a resting tension of $2 \mathrm{mN}$ ) and the amplitudes of both the fast component (about $1.8 \mathrm{mN}$ ) and the slow component of tension increase (about $2.8 \mathrm{mN}$ ) revealed only a slight temperature dependency. Time parameters such as the time constants of the fast $\left(\tau_{1}\right.$ about $0.8 \mathrm{~s}$ at $37^{\circ} \mathrm{C}$ and $2.07 \mathrm{~s}$ at $25^{\circ} \mathrm{C}$ ) and the slow component $\left(\tau_{2}\right.$ about $4.7 \mathrm{~s}$ at $37^{\circ} \mathrm{C}$ and $15.8 \mathrm{~s}$ at $\left.25^{\circ} \mathrm{C}\right)$ are distinctly temperature dependent. The activation energy within the temperature range tested was found to be about $38 \mathrm{~kJ} \mathrm{~mol}^{-1}$ for $\tau_{1}$ and $41 \mathrm{~kJ}$ $\mathrm{mol}^{-1}$ for $\tau_{2}$. During sustained tonic activation, the time constants of postvibration tension recovery changed biphasically whereas the tension parameters remained nearly unchanged. $\tau_{2}$ reached its lowest level after $22 \mathrm{~s}\left(37^{\circ} \mathrm{C}\right)$ or after $34 \mathrm{~s}$ $\left(25^{\circ} \mathrm{C}\right)$ of preceding electrical stimulation. Both the time needed to reach maximum contractility and the time constants of tension recovery were temperature dependent. These findings are consistent with the existence of enzymatic mechanisms controlling smooth muscle contraction kinetics [e.g. activity of the myosin light chain kinase; Aksoy et al., Am. J. Physiol. 242, C109-16(1982)].

\section{Differential effect of cAMP and cGMP in skinned fibres from guinea-pig taenia coli and chicken gizzard}

G. Pfitzer ${ }^{1}$, L. Merkel ${ }^{1}$, J. Wagner ${ }^{1}$, F. Hofmann ${ }^{2}$ and J. C. Rüegg ${ }^{1}$

Departments of ${ }^{1}$ Physiology and ${ }^{2}$ Pharmacology, University of Heidelberg, 6900 Heidelberg, G.F.R. cAMP and cGMP may play a role in smooth muscle relaxation which might partly be mediated by a direct effect on the contractile apparatus. We examined the effect of cGMP- and CAMPdependent protein kinase on submaximal and maximal. contraction in skinned smooth muscle fibres from chicken gizzard, guinea-pig taenia coli and coronary smooth muscle in ATP salt solution under identical experimental conditions. While maximal force development was barely affected, in all cases submaximal development at PCa 5.86 was affected differently: 
$\%$ inhibition of tension at $p$ Ca 5.86 (mean \pm S.E.M.)

\begin{tabular}{lccc} 
& Chicken gizzard & Taenia coli & Pig coronary \\
\cline { 2 - 4 } & & & \\
Control & $3.3 \pm 1.8$ & $4.4 \pm 2.0$ & - \\
$\quad$ cGMP $(5 \mu \mathrm{M})$ & $4.2 \pm 2.0$ & $71.5 \pm 13.0$ & $34.8 \pm 11.2$ \\
$\quad+$ cGMP + cG-kinase $(0.1 \mu \mathrm{M})$ & $17.5 \pm 3.7$ & $100 \%$ & - \\
cAMP-kinase $(0.5 \mu \mathrm{M})$ & $100 \%$ & $100 \%$ & 85 \\
\hline
\end{tabular}

While chicken gizzard myosin light chain kinase was phosphorylated by cAMP-kinase it was not phosphorylated by cGMP-kinase under identical conditions. This may account for the inability of cGMP to inhibit contraction in gizzard. During maximal force development of skinned guinea-pig taenia coli at pCa 4.5 (pH 6.7) the extent of myosin light chain-2 phosphorylation (\% LC-P) increased from $16.4 \%$ (resting value) to $54.8 \pm 3.5 \%$. Reducing $\mathrm{Ca}^{2+}$ to $\mathrm{pCa} 5.86$ reduced force and LC-P to $57.7 \pm 4.5 \%$ and $41.6 \pm 2.2 \%$ respectively in the absence of cAMP, and to $23.8 \pm 4.9 \%$ and $27.6 \pm 4.6 \%(n=5)$ in the presence of cAMP $(0.1 \mathrm{mM})$. Determination of LC-P [Gagelmann et al., J. Musc. Res. Cell Motility 3, 478 (1982)] was performed in fibres fixed with ice-cold TCA while being under tension at $L_{0}$ as phosphorylation was found to be slightly length dependent $[23.4 \pm 2.6 \%$ (8) at $50 \% L_{0}$ and $30.8 \pm 1.9 \%(8)$ in skinned chicken gizzard fibres held at $150 \% L_{\mathrm{o}}$ and $\mathrm{pCa}$ 5.8].

\section{The contractile apparatus of smooth muscle revisited}

J. V.Small ${ }^{1}$ and J. De May ${ }^{2}$

${ }^{1}$ Institute of Molecular Biology, Austrian

Academy of Sciences, Salzburg, Austria

${ }^{2}$ Laboratory of Oncology, Janssen Pharmacentica, Beerse, Belgium

With the aim of clarifying the three-dimensional architecture of the contractile apparatus of smooth muscle we have been applying immunocytochemistry to isolated smooth muscle cells and to ultrathin sections of smooth muscle at the light and electron microscope level. Electron microscope immunocytochemistry has been facilitated by the employment of polyvinyl alcohol as a new water-soluble embedding medium.

In isolated cells in the light microscope antibodies to myosin (SI and $\mathrm{L}_{20}$ light chain) revealed an apparently discontinuous fibrillar pattern whereas antibodies against filamin and desmin labelled continuous fibrils. Actin and tropomyosin showed a nonfibrillar, homogeneous distribution in both whole cells and in thin sections. Vinculin, which is known to be localized in the plasmalemma dense plaques, was shown in whole cells to be distributed in continuous axial 'ribs' on the cell surface. Alpha-actinin was found in a spot pattern consistent with its reported presence in dense bodies but showed only occasional colocalization with the peripheral vinculin ribs.

In the electron microscope, immunogold staining revealed, in cross-sections, a homogeneous distribution of actin, consistent with the presence of actin within dense bodies. Also, antibodies to the head of the myosin molecule labelled nearly all myosin filaments in thin cross-sections, indicating the lack of a central crossbridge-free bare zone on the in vivo thick filaments.

\section{Thin filament regulation in vascular smooth muscle: a $120000 M_{\mathrm{r}}$ regulatory protein}

C. W. J. Smith and S. B. Marston

Cardiothoracic Institute, 2 Beaumont Street,

London W1N 2DX, U.K.

Native thin filaments from vascular smooth muscle (VSM) activate myosin's MgATPase at $\mathrm{Ca}^{2+}$ concentrations of $10^{-4} \mathrm{M}$ but not $10^{-9} \mathrm{M}$, whereas VSM actin and actin tropomyosin activate myosin irrespective of $\mathrm{Ca}^{2+}$ concentration [Marston \& Smith, J. Musc. Cell Motility Res, 5, 559-75 (1984)]. We have separated the three major components of VSM thin filaments (actin, tropomyosin and a $120000 M_{\mathrm{r}}$ protein) and investigated their functional properties. The $120000 M_{\mathrm{r}}$ protein binds to VSM actin with a stoichiometry of 1 per 20-30 actin monomers. In the presence of tropomyosin, the stoichiometry is unchanged but the affinity of binding is increased from $0.3 \times 10^{5} \mathrm{M}$ actin $^{-1}$ to $4.3 \times 10^{5} \mathrm{M}$ actin $^{-1}$. Upon the addition of $\mathrm{Ca}^{2+}$-calmodulin, this binding is partially 
reversed. The $120000 M_{\mathrm{r}}$ protein also interacts with calmodulin-sepharose in a $\mathrm{Ca}^{2+}$-dependent manner. The $120000 M_{\mathrm{r}}$ protein inhibits the activation of myosin's MgATPase by VSM actintropomyosin, but not by actin alone. The inhibition of VSM tropomyosin + skeletal muscle actin by $120000 M_{\mathrm{r}}$ protein can be readily reversed under normal assay conditions $\left(25^{\circ} \mathrm{C}, 60 \mathrm{mM} \mathrm{KCl}\right)$ by $\mathrm{Ca}^{2+}+$ calmodulin. With VSM actin + VSM tropomyosin, however, the assay conditions have to be changed $\left(37^{\circ} \mathrm{C}, 70 \mathrm{mM} \mathrm{KCl}\right)$ before $\mathrm{Ca}^{2+}+$ calmodulin will produce a full relief of inhibition. We conclude that VSM thin filaments are regulated by an inhibition-depression mechanism. The actin-tropomyosin, which together are fully active, are inhibited at resting $\mathrm{Ca}^{2+}$ concentrations by the binding of $120000 M_{\mathrm{r}}$ protein. At higher $\mathrm{Ca}^{2+}$ concentrations, $\mathrm{Ca}^{2+}$-calmodulin interacts with the $120000 M_{\mathrm{r}}$ protein altering its binding to the thin filament and allowing activation of myosin. A similar mechanism has been proposed for the regulation of gizzard actomyosin [Sobue et al., Biomed. Res. 3, 188-96 (1982)]. (Supported by the British Heart Foundation.)

\section{Ca-activation of smooth muscle myosin-kinase-calmodulin complex Apolinary Sobieszek Institute of Molecular Biology. Austrian Academy of Sciences, Billrothstr. 11, Salzburg, Austria}

Vertebrate smooth muscle myosin is normally copurified with high levels of myosin light chain kinase (MLCK) and its activator calmodulin (CM). Their levels are stoichiometric and cannot be detected even with very high loadings of myosin on SDS gels. For fresh myosin preparations the kinase present produced full phosphorylation of the $\mathrm{P}$ light chains within 10-20 s after addition of $\mathrm{Ca}^{2+}$ and MgATP. A major part of the kinase can be removed from myosin using a purification step on Sepharose $4 \mathrm{~B}-\mathrm{CL}$ at high ionic strength and in the absence of divalent cations but the resulting myosin still retains significant levels of the kinase activity (full phosphorylation achieved in 3-6 min). It was shown that this tight association of MLCK and $\mathrm{CM}$ to myosin was independent, Cainsensitive and probably of a hydrophobic nature with apparent $K_{\mathrm{s}}$ values for both subunits of approximately $30 \mathrm{nM}$. Phosphorylation of myosin reduced the binding affinity only approximately two-fold. In separate enzyme kinetic experiments the phosphorylation rates were measured at low and fixed concentrations of the kinase-calmodulin complex while increasing the kinase-apoenzyme concentration to the levels comparable to that of the substrate (kinase-free myosin or isolated $\mathrm{P}$ light chain). Under these conditions the apoenzyme drastically reduced the phosphorylation rates indicating formation of unproductive substrate-enzyme complexes.

These results demonstrate that in smooth muscle there exists an inactive myosin-kinasecalmodulin complex which is activated in the presence of $\mathrm{Ca}^{2+}$ and $\mathrm{MgATP}$. The binding of these two latter ligands to the complex must result in a major conformational change of myosin during which the kinase combines with calmodulin, becomes activated and phosphorylates myosin.

\section{The influence of an actin-modulating protein from smooth muscle on actin-myosin interaction}

Hanna Strzelecka-Golaszewska ${ }^{1}$, Horst Hinssen ${ }^{2}$ and Apolinary Sobieszek ${ }^{2}$

${ }^{1}$ Nencki Institute of Experimental Biology, Polish

Academy of Sciences, Warsaw, Poland

${ }^{2}$ Institute of Molecular Biology, Austrian

Academy of Sciences, Salzburg, Austria

Vertebrate smooth muscle contains an actinbinding modulator protein which affects the polymer state of actin in a $\mathrm{Ca}^{2+}$-sensitive manner [Hinssen et al., FEBS Lett. 166, 90-5 (1984)]. The modulator nucleates $\mathrm{G}$-actin polymerization, caps filament ends, and severs preformed actin filaments. Since the average length of actin filaments in the presence of the modulator is directly dependent on the modulator:actin molar ratio, this protein may serve as a tool to study the influence of actin filament length on various actin-linked processes. In this work we have investigated the influence of the actin modulator from pig stomach (PSAM) on actin-myosin interaction of reconstituted skeletal muscle actomyosin. Two opposite effects of PSAM were observed: (I) stimulation of both the $\mathrm{Mg}^{2+}$-ATPase activity and superprecipitation at low $\mathrm{KCl}$ concentrations, and (2) inhibition of these two interrelated processes at an ionic strength close to physiological. Both effects were $\mathrm{Ca}^{2+}$-dependent, reflecting the requirement for $\mathrm{Ca}^{2+}$ ions for the interaction of PSAM with actin. With acto-SI, inhibition of the actin-activated ATPase activity only was observed, at both low and high $\mathrm{KCl}$ concentrations. The inhibition increased with increasing PSAM to actin ratio and at a $1: 2$ molar ratio practically no actin-activation was observed indicating that the $1: 2$ PSAM-actin complex does not act as a cofactor for the myosin ATPase. In contrast, the stimulation observed with 
intact filamentous myosin resulted from a more favourable steric organization of the shorter actin filaments within the actomyosin gel. This latter effect suggests that the often observed actin-linked $\mathrm{Ca}^{2+}$-dependent activation of the actomyosin ATPase by smooth muscle crude extracts could be due to a Ca-dependent shortening of actin filaments by the modulator which is normally present in such extracts.

\section{Calmodulin-induced contraction of 'native' skinned chicken gizzard is not associated with phosphorylation of myosin light chain (LC-2) \\ J. Wagner \\ Department of Physiology. University of \\ Heidelberg, 6900 Heidelberg, G.F.R.}

According to the phosphorylation hypothesis, there is a close correlation between myosin light chain-2 phosphorylation and smooth muscle contraction. Here we report that contraction of skinned fibres of chicken gizzard is not obligatorily associated with an increase in LC-2 phosphorylation which was determined according to Gagelmann et al. [J. Musc. Res. Cell Motility 3, 478 (1982)]. Detergent skinned chicken gizzard prepared and investigated as soon as possible after slaughtering did not produce force in ATP salt solution at $\mathrm{pCa} 5.8$ but contracted maximally at pCa 5.2. At pCa 5.8 addition of low $(0.05 \mu \mathrm{M})$ concentrations of calmodulin (CaM) caused a nearly half maximal contraction within 20 min whereas the extent of LC-2 phosphorylation remained as low as in CaM-free solution in which the fibre was relaxed. When, at the same $\mathrm{pCa}, \mathrm{CaM}$ concentration was increased to $0.1 \mu \mathrm{M}$, tension development was nearly maximal while phosphorylation still remained at basal levels. A further increase in CaM concentration (to $0.5 \mu \mathrm{M}$ ) stimulates LC-2 phosphorylation without changing force drastically:

\begin{tabular}{ccccc}
\hline $\begin{array}{c}\text { Prepar- } \\
\text { ation }\end{array}$ & $\mathrm{pCa}$ & $\begin{array}{c}\mathrm{CaM} \\
(\mu \mathrm{M})\end{array}$ & $\begin{array}{c}\text { Force } \\
(\% \text { max. })\end{array}$ & $\begin{array}{c}\text { LC-2 } \\
\text { phosphorylation } \\
(\% \mathrm{LC}-2 \text { total })\end{array}$ \\
\hline & & & & \\
$\mathrm{N}$ & 5.8 & 0 & 0 & $8.2 \pm 1.3$ \\
$\mathrm{~N}$ & 5.8 & 0.05 & $46.6 \pm 3.8$ & $8.7 \pm 1.1$ \\
$\mathrm{~N}$ & 5.8 & 0.1 & $80.4 \pm 4.5$ & $11.9 \pm 1.5$ \\
$\mathrm{~N}$ & 5.8 & 0.5 & $96.1 \pm 0.8$ & $29.6 \pm 1.4$ \\
$\mathrm{~S}$ & 5.8 & 0 & $62.4 \pm 4.9$ & $42.6 \pm 3.4$ \\
\hline
\end{tabular}

Values given are mean \pm S.E.M. of $4-7$ determinations; $N$, native preparations; $S$, stored fibres.

In contrast to 'native' preparations, fibres stored at $-20^{\circ} \mathrm{C}$ in glycerol ATP salt solution up to two months contracted at $\mathrm{pCa} 5.8$ even in the absence of exogenous calmodulin. During contraction LC -2 phosphorylation increased from 12 to $42.6 \pm 3.4 \%(n=5)$. We suggest that in 'native', but not in aged preparations, force development may be inhibited by a factor which can be 'neutralized' by CaM.

\section{Regulation of actomyosin ATPase by a single calcium-binding site on troponin $\mathrm{C}$ from crayfish}

W. Wnuk, M. Schoechlin and E. A. Stein

Department of Biochemistry, University of

Geneva, P.O. Box 78 Jonction, 1211 Geneva 8,

\section{Switzerland}

In crayfish, troponin C contains only one Cabinding site with an affinity in the range of physiological free $\mathrm{Ca}^{2+}\left(K=2 \times 10^{5} \mathrm{M}^{-1}\right)$. This site is analogous to the $\mathrm{Ca}^{2+}$-specific sites of vertebrate TnC since it does not bind $\mathrm{Mg}^{2+}$ at physiological levels of $\mathrm{Mg}^{2+}$. Hence, the crayfish tropomyosintroponin complex with the simplest physiologically significant $\mathrm{Ca}^{2+}$-binding properties constitutes a convenient system to study $\mathrm{Ca}^{2+}$ regulation. In the complexes of troponin $\mathrm{C}$-troponin I, troponin and troponin-tropomyosin, the regulatory Ca-specific site exhibits a $10-20$-fold higher affinity $\left(K=2-4 \times 10^{6} \mathrm{M}^{-1}\right)$. The latter affinity is reduced to that of troponin $C$ upon incorporation of the troponin-tropomyosin complex into the actin filament. The Ca-binding constant is again shifted to a higher value $\left(7 \times 10^{6} \mathrm{M}^{-1}\right)$ when regulated actin is associated with nucleotide-free myosin. In contrast, during steady-state ATP hydrolysis, regulated actomyosin exhibits Ca-binding properties ( $K=$ $3 \times 10^{5} \mathrm{M}^{-1}$ ) similar to those of regulated actin without myosin. Both crayfish myofibrils and rabbit actomyosin regulated by crayfish troponintropomyosin display a steep rise in ATPase activity with $\left[\mathrm{Ca}^{2+}\right]$. Both the position and slope of the $\mathrm{pCa} / \mathrm{ATPa}$ a curve are similar to those of myofibrils from rabbit skeletal muscle, whose troponin $\mathrm{C}$ has four Ca-binding sites. This rules out a mechanism which requires more than one site occupied by $\mathrm{Ca}^{2+}$ on troponin $\mathrm{C}$ to explain the steep responses to $\mathrm{Ca}^{2+}$. Comparison of the $\mathrm{pCa} / \mathrm{ATP}$ ase relationship and the Ca-binding properties for the crayfish troponin-regulated actomyosin indicates that while the threshold 
$\left[\mathrm{Ca}^{2+}\right]$ for activation corresponds to the range of $\left[\mathrm{Ca}^{2+}\right]$ where the regulatory site in its low affinity state $\left(K=1 \times 10^{5} \mathrm{M}^{-1}\right)$ starts to bind $\mathrm{Ca}^{2+}$ significantly, full activation is reached at $\left[\mathrm{Ca}^{2+}\right]$ for which the Ca-specific site in its affinity state $\left(K=3 \times 10^{6} \mathrm{M}^{-1}\right)$ approaches saturation. These results suggest that, in the actomyosin ATPase cycle, there are at least two calcium-activated states of regulated actin (one low and one high), the high affinity state being induced by interactions of myosin with actin in the cycle.

\section{Rat uterine smooth muscle studied by ${ }^{31} \mathrm{P}$ nuclear magnetic resonance (n.m.r.) spectroscopy \\ S. Wray and M. J. Dawson \\ Physiology Department, University College}

London, Gower Street, London WC1E 6BT, U.K.

${ }^{31} \mathrm{P}$ n.m.r. spectroscopy detects phosphoruscontaining metabolites in living tissue e.g. ATP, $\mathrm{PCr}$ and inorganic phosphate $\left(\mathrm{P}_{\mathrm{i}}\right)$. n.m.r. has been widely applied to the study of skeletal and cardiac muscle energetics [see e.g. Dawson \& Wilkie, Recent Adv. Physiol. 10, 247-76 (1984) for a review]. We and others have recently started applying ${ }^{31} \mathrm{P}$ n.m.r. to the study of uterine smooth muscle [e.g. Dawson \& Wray, ]. Physiol., Lond. 336, 19-20P (1983)]. Rat uteri from non-pregnant, pregnant and post-partum animals have been excised, slit open and superfused with de-Jalons solution at $\mathrm{pH} 7.2$, and bubbled with $95 / 5 \%$ $\mathrm{O}_{2} / \mathrm{CO}_{2}$ whilst in the n.m.r. spectrometer. The studies were carried out at 4 or $20^{\circ} \mathrm{C} .{ }^{31} \mathrm{P}$ n.m.r. spectra were obtained from 500-800 radiofrequency pulses, at $2 \mathrm{~s}$ intervals and $30 \mu \mathrm{s}$ duration at $81 \mathrm{MHz}$ frequency.

The ratio of PCr to ATP was found to be higher in the pregnant uterus, $0.95 \pm 0.05$ (mean \pm S.E.M. $n=7$ ), than the non-pregnant uterus $0.76 \pm 0.06$ $(n=7, P<0.05)$. The ratio of $\mathrm{PCr} / \mathrm{ATP}$ found was more than double that found previously by chemi$\mathrm{cal}$ analysis, 0.42, [Walaas \& Walaas, Acta physiol. scand. 21, 1-17 (1950)]. This difference is probably due to breakdown of $\mathrm{PCr}$ during extraction for chemical analysis in the earlier studies. After parturition the ratio of $\mathrm{PCr} / \mathrm{ATP}$ returns to nonpregnant values within $24 \mathrm{~h}$ : There is an increase in inorganic phosphate content in the post-partum uterus compared to either pregnant or nonpregnant animals; $\mathrm{P}_{\mathrm{i}}$ /ATP 0.86 compared to 0.42 and 0.37 respectively. These changes in highenergy phosphorus metabolites are consistent with the energetic demands on the uterus during parturition. It is as yet unknown how the mechanical performance of uterine muscle is related to changes in metabolite concentrations. (This work was supported by Action Research The National Fund for Research into Crippling Diseases. S.W. is grateful for a travel grant from the Wellcome Trust.)

\section{MOTILITY IN MUSCLE AND NONMUSCLE CELLS}

\section{Modulation of the microfilament system in nonmuscle cells by microinjection}

A. Füchtbauer and B. M. Jockusch

Developmental Biology Unit, W7, University of

Bielefeld, POB 8640, D-4800 Bielefeld 1, G.F.R.

The microfilament organization and its correlation with cellular adhesion was analysed by injecting actin-binding proteins and specific antibodies into tissue culture cells, using the Graessmann technique [Graessmann \& Graessmann, Proc, natn. Acad. Sci. U.S.A. 73, 366-70 (1976)]. Capping proteins from brain and from an acellular slime mould which interfere with growth of filamentous actin at the positive end were injected at concentrations of $0.002-2.0 \mathrm{mg} \mathrm{ml}^{-1}$. They were found to disrupt stress fibres as well as focal adhesion plaques (FC) in fibroblasts and epithelial cells [Füchtbauer et al.,
Nature, Lond. 304, 361-4 (1983)]. Stress fibres disintegrated first in their distal parts concomitant with FC. Consequently, cells rounded up and contracted. Gelsolin from pig plasma [Harris et al., Analyt. Biochem. 119, 102-14 (1982)] and actin depolymerizing factor (ADF) from human serum [Chaponnier et al, Experientia 35, 1039-41 (1979)] which both sever actin filaments in vitro also caused disintegration of stress fibres very efficiently, starting from the (perinuclear) injection area.

Microinjection of specific anti-actin had no effect on microfilament organization. Microinjection of specific antivinculin, however, led to weakening and subsequent disappearance of $\mathrm{FC}$, followed by disintegration of stress fibres starting at their distal ends. However, cells did not contract 
to the same degree as with capping proteins, and vinculin was found to remain at the plasma membrane [Jockusch \& Füchtbauer, Cell Motility 3, 391-7 (1983); Füchtbauer \& Jockusch, J. submicrosc. Cytol. 16, 109-10 (1984)].

The effect of all these proteins on stress fibres was concentration dependent. While the disruptive effects of injected capping proteins, gelsolin and ADF were fully reversible, anti-vinculin injected cells did not restore focal adhesion plaques or stress fibres. In contrast to the severing proteins, capping proteins acted only on stress fibres in the living cell, indicating that they interfered with an equilibrium between filamentous actin and smaller subunits. (This work is supported by the Deutsche Forschungsgemeinschaft and the Stiftung Volkswagenwerk.)

\section{Lysosome movements studied by cytomagnetometry \\ P. Gehr, J. D. Brain and S. B. Bloom \\ Department of Anatomy, University of Berne, \\ Berne, Switzerland and Department of \\ Environmental Science and Physiology, Harvard \\ School of Public Health, Boston, U.S.A.}

Microgram quantities of submicrometric ferrimagnetic particles instilled into hamster lungs are phagocytized by alveolar macrophages. A magnetic field external to the body magnetizes and aligns these particles. Upon removal of this magnetizing field the particles produce a remanent magnetic field which is measurable above the chest with a fluxgate magnetometer. The remanent field is, however, not stable but decays with time. This process is called relaxation and is caused by random progressive rotation of the particles away from their initial aligned state. This results in a decreased vector sum of the magnetic field measured at the body surface. Electron microscopy of alveolar macrophages showed that the ingested magnetic particles were contained in phagosomes or secondary lysosomes. These organelles are an integral part of the cytoskeleton and can be moved by its contractile elements much like puppets manipulated by their strings. Magnetometric measurements of monolayer cultures of alveolar macrophages, containing magnetic particles, demonstrated the same phenomenon; again, after an exfernal magnetizing field was removed, lysosome motion caused a gradual decrease of the remanent field measured above the cells. The addition of $20 \mu \mathrm{M}$ cytochalasin $\mathrm{B}$ and $100 \mu \mathrm{M}$ p-trifluoromethoxy carbonyl cyanide phenylhydrazone to cultures kept at $37^{\circ} \mathrm{C}$, or cooling the cultures to $20^{\circ} \mathrm{C}$, resulted in a significantly slower relaxation, whereas colchicin scarcely influenced this. No relaxation was observed after fixing the culture with glutaraldehyde. From these findings it can be concluded that relaxation is a vital process which is energy dependent and is caused by intracellular forces imparted on lysosomes, containing magnetic particles, by contrasting filaments. The cytomagnetometric measurements provide evidence that these filaments are actin and, therefore, that lysosome motion is an actin-based mechanism. (Supported by SNSF grant 3.130.081, NIH grant \# HL-31029.)

\section{Characterization and function of an antibody against smooth muscle myosin microinjected in tissue culture cells}

B. Höner and B. M. Jockusch

Developmental Biology Unit, University of

Bielefeld, POB 8640, D-4800 Bielefeld 1, G.F.R.

Highly purified chicken smooth muscle (gizzard) myosin was used as an antigen to raise antibodies in specific pathogen-free rabbits. Specific immunoglobulin was purified from serum on myosin-nitrocellulose. In western blots, sera as well as specific immunoglobulins were shown to react only with the heavy chain of gizzard myosin but not with skeletal muscle myosin. In rotary shadowing experiments, it was demonstrated that the specific antibodies reacted with various regions in the tail portion of the myosin molecule. In indirect immunofluorescence, the antibody stained stress fibre myosin in tissue culture cells in the expected pattern [Weber \& Groeschel-Stewart, Proc, natn. Acad. Sci. U.S.A. 71, 456I-4 (1976)]. When the antibody was microinjected into PtK 2 cells using the Graessmann technique [Graessmann \& Graessmann Proc. natn. Acad. Sci. U.S.A. 73, 366-70 (1976)], cellular myosin was found to leave its position within the stress fibres, while F-actin remained unaltered. This effect was transient: $12 \mathrm{~h}$ after microinjection, cells had fully recovered and expressed myosin-containing stress fibres indistinguishable from controls. (This work was supported by the Deutsche Forschungsgemeinschaft and the Stiftung Volkswagenwerk.)

\section{A 40000 dalton protein from bovine brain restricts the polymer state of actin in a $\mathrm{Ca}^{2+}$-dependent manner \\ A.-C. Hurny and W. Wnuk \\ Department of Biochemistry, University of Geneva, P.O. Box 78 Jonction, 1211 Geneva 8. Switzerland}

A protein preparation has been obtained from 
bovine brain extract that modulates the state of actin filaments in a $\mathrm{Ca}^{2+}$-dependent manner. The purification procedure included chromatography on DEAE- and CM-cellulose, hydroxylapatite and Sephacryl S-200. The actin-modulating activity coincided with a 40000 dalton polypeptide, as found by both electrophoresis in SDS polyacrylamide gels and molecular exclusion chromatography. When added either to preformed F-actin filaments or to G-actin, prior to polymerization, the modulator reduced the low-shear apparent viscosity of actin filaments, with halfmaximal reduction occurring at a free $\mathrm{Ca}^{2+}$ concentration of $40 \mathrm{nM}$. The modulator was also a potent inhibitor of actin filament elongation provided that $\mathrm{Ca}^{2+}$ was present. These results suggest that this protein may be involved in the control of actin filament assembly and interaction in the nervous tissue.

\section{Brevin, a calcium-dependent actin serving protein from serum \\ M. C. Kilhoffer ${ }^{1}$, Y. Mely ${ }^{1}$, G. Isenberg ${ }^{2}$ and \\ D. Gerard ${ }^{1}$ \\ ${ }^{1}$ Laboratoire de Physique, UA CNRS 491, Faculté \\ de Pharmacie, B.P. No.10, 67048 Strasbourg \\ Cédex, France \\ ${ }^{2}$ Max Planck Institute, Department of Neurochemistry,} Am Klopferspitz 18a, 8033 Martinsried, Munich, G.F.R. Brevin has been isolated from serum as a high molecular weight (92 000) calcium-binding protein able to interact with actin filaments. Calcium binding to brevin induces a conformational change which is suggested by the behaviour of the protein during hydrophobic chromatography as well as by changes in spectroscopic parameters (namely fluorescence polarization).

The effect of brevin on actin polymerization has been investigated using both viscometry and fluorescence of pyrene-labelled actin. In the presence of $\mathrm{Ca}^{2+}$, brevin, even at very low ratios to actin, strongly accelerates the initial steps of actin polymerization probably by stabilizing actin nuclei and decreases the final viscosity of actin samples by reducing the average filament length. Data obtained by fluorescence measurements also indicate that brevin increases the rate of monomer incorporation into the actin filament. Filamentsevering activity of brevin could only be observed in the presence of $\mathrm{Ca}^{2+}$, whereas capping of one of the filament ends seems to occur both in the presence and absence of $\mathrm{Ca}^{2+}$. Direct evidence for severing and capping activities of brevin in the presence and absence of $\mathrm{Ca}^{2+}$ could be obtained respectively by fluorescence microscopy of astrocytes Triton models and electron microscopy of $\mathrm{S}_{1}$-decorated actin filaments.

\section{Structure of the contractile protein of the bacteriophage $\mathrm{T} 4$}

B. F. Poglazov and I. I. Serysheva

A. N. Bach Institute of Biochemistry,

U.S.S.R. Academy of Sciences, Moscow, U.S.S.R.

The contractile sheath of bacteriophage T4 is the simplest motile apparatus and is composed of a single protein, the product of gene I8 (PG 18). This protein was isolated in the dissociated, electrophoretically homogeneous and biologically active state. Using electron microscopy it was found that there are two stages in the polymerization of PG 18 , i.e. band helices and polysheaths. In accordance with this the possibility of dynamic equilibrium in the cell between the monomers of PG 18, polysheaths and extended sheaths is discussed. On the basis of the circular dichroism spectra, the contents of the secondary elements were calculated for the monomer PG 18, helices, polysheaths and contractile sheaths. It is shown that the sheath contraction results in a change of the secondary structure of PG 18, i.e. an increase of the $a$-helix conformation and a larger proportion of the unordered structure and $\beta$-form are observed.

\section{Platelet tropomyosin: properties and comparison with its skeletal muscle counterpart}

G. Prulière, E. der Terrossian and A. d'Albis

Laboratoire de Biologie Physicochimique,

Université Paris-Sud, 91405 Orsay, France

Tropomyosin represents as much as $2 \%$ of the total proteins in pig platelet [Terrosian et al., J. molec. Biol. 153, 147-67 (1981)]. Contrary to the muscle protein, it contains only one type of subunit $[a$ type, according to Kardamy \& Fiszman, FEBS Lett. $163,250-6$ (1983)]; we present evidence that the second band which often appears on SDS gel after storage does not bind to platelet actin and can be considered as a denatured form. The association of tropomyosin with platelet actin has been investigated under different conditions of salt and magnesium: at physiological salt conditions (0.I M $\mathrm{KCl}$ and $2 \mathrm{mM} \mathrm{Mg}^{2+}$ ), binding of $1 \mathrm{~mol}$ tropomyosin to $6 \mathrm{~mol}$ actin was observed, when the tropomyosin is added in two-fold excess. Electron microscopy and viscosity measurements indicate that platelet tropomyosin, like the striated muscle protein, is able to self associate and polymerizes to form fibres at low ionic strength. 
Tactoids paracrystals obtained in the presence of $\mathrm{Mg}^{2+}, \mathrm{Ca}^{2+}$ or $\mathrm{Ba}^{2+}$ exhibit similar patterns with a $345 \AA$ repeat. For the first time, we show that a pure nonmuscle tropomyosin can form nets paracrystals. Moreover, association of tropomyosin with muscle troponin I and brain calmodulin induces the hexagonal net structure described with the striated [Caspar et al., J. molec. Biol. 41, 87-107 (1969)] or smooth muscle tropomyosins [Ditgens et al., J. Musc, Res. Cell Motility 3, 57-76 (1982)]. Relations between the sequence of horse platelet tropomyosin [Lewis et al., FEBS Lett. 156, 269-73 (1983)] and paracrystals patterns, with or without muscle troponin, are under investigation.

\section{Desmin filaments in in vivo and in vitro growing rat heart myocytes}

L. Rappaport, B. Bertier-Savalle, B. Escoubet, F. Marotte and J. L. Samuel

INSERM U 127, Hopital Lariboisière, 41 Bd de la

Chapelle, 75010 Paris, France

Distribution of desmin filaments was studied, by immunofluorescence labelling, in growing myocytes. (I) In vivo the cells were isolated from four-week-old rat heart, the growth of which was stimulated either by mechanical overload induced by aortic stenosis or by injection of thyroxine (T4) into hypothyroid rats; (2) in vitro the myocytes were isolated from three-day-old rat and cultured.

In both in vivo models, rod-shaped myocytes exhibited no variations in the distribution of the intermediate filaments and the cellular concentration of desmin was unchanged. In the same time, however, as we have previously shown, the network of the microtubules was reorganized in most of these cells [reviewed in Samuel et al., Eur. ]. Cell. Biol, (in press)].

In the series of in vitro experiments, cultured myocytes underwent changes in shape with spreading and myofibrils were no longer oriented in parallel. A dramatic change in the distribution of the intermediate filaments was observed in these conditions. Desmin, which remained associated with the intercalated disks but no longer to the $Z$ lines, displayed as foci randomly oriented throughout the sarcoplasm. In both in vivo and in vitro experiments the electrophoretic properties of desmin were unchanged.

We conclude that the synthesis of desmin in growing myocytes is closely related to that of the sarcomeric proteins. However the cross-striated appearance of intermediate filaments seems closely dependent on the organization in register of the myofibrils.
Immunological evidence for the presence of spectrin, protein 4.1 and ankyrin-like proteins in the cytoskeleton isolated from thyroid plasma membranes: characterization of thyroid spectrin

F. Regnouf, E. Nguyen, G. Chétrite, R. Cassoly and L. A. Pradel

Institut de Biologie Physico-Chimique, 13 rue

Pierre et Marie Curie, 75005 Paris, France

The cytoskeleton of thyroid cells is concentrated in the apical area of the cell that is in contact with follicular lumen where exocytosis and endocytosis take place under TSH stimulation.

Morphological studies performed on purified thyroid apical plasma membrane fractions, using HMM decoration, have shown a submembranous cytoplasmic network of actin microfilaments attached to the plasma membrane, at tight junctions but also on vesicles encaged in the network. Treatment of these fractions by the nonionic detergent Triton $X-100$, provided an insoluble material composed of four major polypeptides: a doublet of highmolecular weight and three bands of 200000 , 100000 and 43000 . The last three have been characterized as myosin, $a$ actinin and actin, respectively.

Since high molecular weight proteins, composed of two nonidentical polypeptides have been demonstrated as spectrins in a great number of cell types and tissues, we have investigated the presence in our thyroid membrane fractions of polypeptides immunologically related to spectrin and also to the spectrin-associated proteins that are involved in human erythrocyte cytoskeletonmembrane interactions (ankyrin, protein 4.1, band 3).

The results demonstrated that polypeptides sharing common antigenic determinants with erythrocyte and brain spectrin, erythrocyte ankyrin and protein 4.1 are present in thyroid membrane fractions. On the other hand, thyroid spectrin has been purified from hog thyroid gland in milligram quantities. It is composed of two polypeptides of 240000 and 235000 and in this respect is similar to brain spectrin. Although thyroid cells are epithelial polarized cells and thereby show some morphological features of their cytoskeleton similar to those of intestinal epithelial cells, they do not contain the spectrin analogue TW $260000 / 240000$ found in these cells.

Thyroid spectrin binds calmodulin on its $240000 \mathrm{~K}$ subunit, in a $\mathrm{Ca}^{2+}$-dependent manner. It does cross-link F-actin as already demonstrated for the spectrins isolated from other cells and tissues. 
Characterization of antibodies against mammalian profilin

Magdalena Segura, Lars Nilsson, Odd Nygård and Uno Lindberg

Wenner-Gren Institute, University of Stockholm,

Norrtullsgatan 16, S-113 45 Stockholm, Sweden

Antibodies against calf spleen profilin were raised in rabbits. Total IgGs from hyperimmune rabbits were prepared by affinity chromatography on a protein A-Sepharose column. Affinity-purified profilin IgGs were selected from total IgGs on a profilin-Sepharose column. One population of profilin antibodies were eluted with $0.2 \mathrm{M}$ glycine $\mathrm{pH}$ 2.7 and another population of antibodies came out with subsequent elution with $5 \mathrm{M}$ guanidine- $\mathrm{HCl}$. The specificity of the antibodies was tested by immunostaining of proteins electrophoretically transferred to nitrocellulose paper from SDS polyacrylamide gels. Samples tested were crude extracts of calf spleen human skin fibroblasts and human U 698 lymphoma cells, purified profilin from calf spleen human platelets and human placenta. In all cases profilin was the most strongly stained band, using both total IgGs and affinitypurified profilin antibodies. The localization of profilin in cells was examined by indirect immunofluorescent staining of fixed cells. The results suggested that profilin is present throughout the cell except for the nucleus.

Interaction of calmodulin and its fragments with erythrocyte membrane $\mathrm{Ca}^{2+}$-ATPase W. Szyja, A. Wrzosek, H. Brzeska, G. Boguta and M. G. Sarzała

Nencki Institute of Experimental Biology,

Polish Academy of Sciences, 3 Pasteur Street, 02-093 Warszawa, Poland

The aim of the work was to investigate whether calmodulin fragments obtained as a result of controlled trypsin digestion of calmodulin, are able to bind calmodulin-dependent enzymes. All experiments were performed on $\mathrm{Ca}^{2+}$-ATPase from erythrocyte membranes which was previously shown to be stimulated to a certain extent by calmodulin fragments.

The purified $\mathrm{Ca}^{2+}$-ATPase was applied to a series of affinity columns obtained by coupling calmodulin fragments to $\mathrm{CNBr}$-activated Sepharose $4 \mathrm{~B}$. It was shown that in the presence of calcium a part of the applied protein was retained on the column. This protein was subsequently eluted by $2 \mathrm{mM}$ EGTA and was shown to reveal $\mathrm{Ca}^{2+}$-ATPase activity.

The amount of $\mathrm{Ca}^{2+}$-ATPase retained on affinity column was very similar in the case of both
TR-C fragments of calmodulin. On the other hand these fragments differ in their ability to activate this enzyme; fragment $\mathrm{TR}_{2}-\mathrm{C}$ is several times more effective in activating $\mathrm{Ca}^{2}+$-ATPase than fragment $\mathrm{TR}_{\mathrm{I}}-\mathrm{C}$. Fragment $\mathrm{TR}_{3}-\mathrm{E}$, which does not activate $\mathrm{Ca}^{2+}$-ATPase, is nevertheless able to bind this enzyme although with an affinity 5-10 times lower than TR-C fragments.

On the basis of these results we postulate that the binding of calmodulin fragment to a calmodulin-dependent enzyme is not sufficient for exerting an activation effect as can be seen in the case of $\mathrm{TR}_{3}-\mathrm{E}$ fragment. Furthermore the affinity of binding does not seem to be correlated with the ability of activating $\mathrm{Ca}^{2+}$-ATPase as can be concluded from the differences in binding and activation between TR-C fragments.

\section{The lipid composition of erythrocyte ghosts from a paramyotonia patient: a biochemical and biophysical study}

G. Szymańska ${ }^{1}$, A. Marx ${ }^{2}$, I. Melzner ${ }^{2}$, M. G. Sarzała ${ }^{1}$ and R. Rüdel ${ }^{3}$

${ }^{1}$ Department of Muscle and Motile Systems

Research, Nencki Institute, Warsaw, Poland

${ }^{2}$ Ableilung für Pathologie and ${ }^{3}$ Abteilung für

Allgemeine Physiologie, Universität Ulm, G.F.R.

Paramyotonia congenita is a genetic disorder inherited as an autosomal dominant trait. We investigated the lipid composition, the cholesterol content, and changes in the fluorescence of pyrendecanoic acid, of the erythrocyte membrane from a paramyotonia patient. The total content of phospholipids, as well as the amount of their respective classes, did not differ significantly from control, except that the sphingomyelin content was lower. The cholesterol content was nearly the same as described in the literature. Significant changes were found in the fatty acid composition of the individual classes of the phospholipids. The content of saturated fatty acids (16:0, 18:0) was increased and the content of unsaturated fatty acids (18:1, 18:2, 18:3,20:4) was decreased. This tendency concerned all phospholipid classes. This alteration in the composition of phospholipids and in the membrane fluidity, measured directly with a fluorescent dye, could be related to the pathomechanism in paramyotonia congenita in which case it is not restricted to erythrocyte membranes but also to other membraneous systems in various tissues.

This is the first report of an alteration of a nonmuscular membrane in this very rare muscle disease. 